\title{
Shedding light on oropharyngeal dysphagia in myotonic dystrophy type 1
}

\author{
Citation for published version (APA):
}

Pilz, W. (2018). Shedding light on oropharyngeal dysphagia in myotonic dystrophy type 1. [Doctoral Thesis, Maastricht University]. Datawyse / Universitaire Pers Maastricht. https://doi.org/10.26481/dis.20180329wp

Document status and date:

Published: 01/01/2018

DOI:

10.26481/dis.20180329wp

Document Version:

Publisher's PDF, also known as Version of record

\section{Please check the document version of this publication:}

- A submitted manuscript is the version of the article upon submission and before peer-review. There can be important differences between the submitted version and the official published version of record. People interested in the research are advised to contact the author for the final version of the publication, or visit the DOI to the publisher's website.

- The final author version and the galley proof are versions of the publication after peer review.

- The final published version features the final layout of the paper including the volume, issue and page numbers.

Link to publication

\footnotetext{
General rights rights.

- You may freely distribute the URL identifying the publication in the public portal. please follow below link for the End User Agreement:

www.umlib.nl/taverne-license

Take down policy

If you believe that this document breaches copyright please contact us at:

repository@maastrichtuniversity.nl

providing details and we will investigate your claim.
}

Copyright and moral rights for the publications made accessible in the public portal are retained by the authors and/or other copyright owners and it is a condition of accessing publications that users recognise and abide by the legal requirements associated with these

- Users may download and print one copy of any publication from the public portal for the purpose of private study or research.

- You may not further distribute the material or use it for any profit-making activity or commercial gain

If the publication is distributed under the terms of Article $25 \mathrm{fa}$ of the Dutch Copyright Act, indicated by the "Taverne" license above, 
Shedding light on

\section{oropharyngeal dysphagia in} myotonic dystrophy type 1

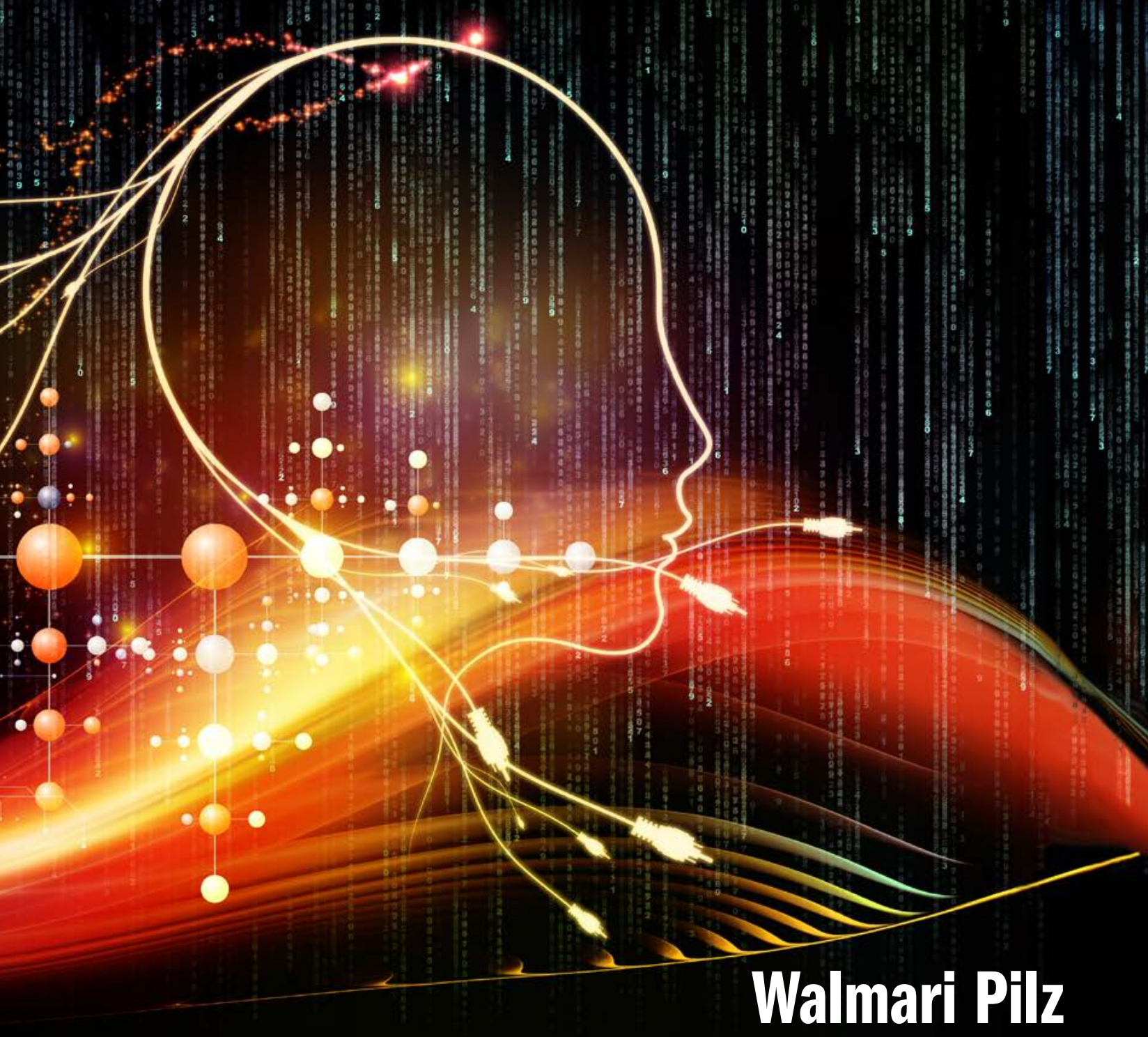


(C) Walmari Pilz, Maastricht 2018

Layout: Tiny Wouters

Production: Datawyse | Universitaire Pers Maastricht

ISBN: 9789461598073

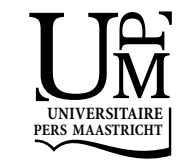

Financial support for the publication of this thesis was kindly provided by:

Fresenius Kabi Netherlands BV, Atos Medical, MediTop Medical Products BV. 


\section{Shedding light on oropharyngeal dysphagia in myotonic dystrophy type 1}

\section{Dissertation}

To obtain the degree of doctor at Maastricht University, on the authority of the Rector Magnificus,

Prof. dr. Rianne M. Letschert

in accordance with the decision of the board of Deans, to be defended in public on Thursday, $29^{\text {th }}$ of March 2018 at 12:00

by

Walmari Pilz

Born in Santa Rosa, Brazil, on November $11^{\text {th }} 1965$ 
Supervisor

Prof. dr. B. Kremer

\section{Co-supervisors}

Dr. L.W.J. Baijens

Dr. V. Lima Passos

\section{Assessment committee}

Prof. dr. S.G.F. Robben (chairman)

Prof. dr. P. Clave, Universitat Autònoma de Barcelona, Spain

Dr. L. van der Molen, Netherlands Cancer Institute - Antoni van

Leeuwenhoek, Amsterdam

Prof. dr. J. Vermeulen 


\section{Table of contents}

$\begin{array}{lll}\text { Chapter } 1 \text { General introduction and outline of the thesis. } & 7\end{array}$

Chapter 2 Oropharyngeal dysphagia in myotonic dystrophy type 1:

a systematic review.

Dysphagia. 2014;29(3):319-31.

Chapter 3 Swallowing assessment in myotonic dystrophy type 1 using fiberoptic endoscopic evaluation of swallowing (FEES). Neuromuscul Disord. 2014;24(12):1054-62.

Chapter 4 Swallow-related quality of life and oropharyngeal dysphagia in myotonic dystrophy.

Submitted: Under revision

Chapter 5 Observers' agreement on measurements in fiberoptic endoscopic evaluation of swallowing.

Dysphagia. 2016;31(2):180-7.

Chapter 6 Identifying patterns of FEES-derived swallowing trajectories using group-based trajectory model.

Dysphagia. 2015;30(5):529-39.

Chapter 7 Summary, general discussion, and future perspectives

Nederlandse samenvatting

Valorization

Acknowledgements

Curriculum vitae

List of publications 

Chapter 1

General introduction 



\section{Myotonic dystrophy type 1}

Myotonic dystrophy, also known as Steinert disease or dystrophia myotonica, was first described as a specific condition in 1909 by Steinert and colleagues. To improve differentiation of the types of dystrophy, the International Myotonic Dystrophy Consortium recommended a new nomenclature in 1999: myotonic dystrophy type 1 (DM1). ${ }^{1}$

DM1 is a dominant inherited disorder and the most common type of muscular dystrophy in adults. The prevalence is estimated at 1 in 8,000 (12.5 per 100000) worldwide with some regions reporting higher prevalence, for instance the Istria region in Croatia (18.1 per 100000 ) and the Saguenay region in Canada $\left(210\right.$ per 100,000). ${ }^{2-4}$ Data collected from 2004 to 2010 at eight (university) medical centers in the Netherlands registered 1117 patients with myotonic dystrophy. ${ }^{5}$ Diagnosis of the disease is based on the family history, clinical examination, and genetic testing. Age of onset, severity of disease, and symptoms present a wide variability, and DM1 has been described as the most variable inherited human disorder. ${ }^{6}$

\section{Genetics}

The mutation causing DM1 results from an expansion of a cytosine-thymine-guanine (CTG) trinucleotide repeat in the 3' untranslated region of the myotonic dystrophy protein kinase (DMPK) gene, located in chromosome 19q13.3..$^{7-9}$ The number of CTG trinucleotide repeats in the DMPK gene can vary. Healthy subjects have between 5 and 37 CTG repeats while in patients with DM1 the number ranges from 50 to 4000 (Figure 1.1). ${ }^{10} \mathrm{~A}$ larger repeat extension is moderately correlated to an earlier age of onset and more severe symptoms. Based on the age of onset, four DM1 phenotypes can be distinguished: (1) congenital type; (2) childhood onset type; (3) adult onset type; and (4) late onset type (mild). ${ }^{6}$ The adult onset type is the most prevalent form. Only patients with the adult type and late onset type were included in the studies presented in this thesis. The size of the CTG repeat, in blood, showed a correlation with disease severity, i.e., patients with a higher CTG repeat expansion have an earlier onset and more severe clinical manifestations. ${ }^{1}$ On the other hand, the degree of muscular weakness does not correlate with the CTG repeats in muscle tissue. ${ }^{11}$ Moreover, CTG repeat size cannot solely be used to distinguish the DM1 types as CTG repeat length overlaps between phenotypes, is not constant, may vary between tissues, and it increases with age. ${ }^{10}$ 


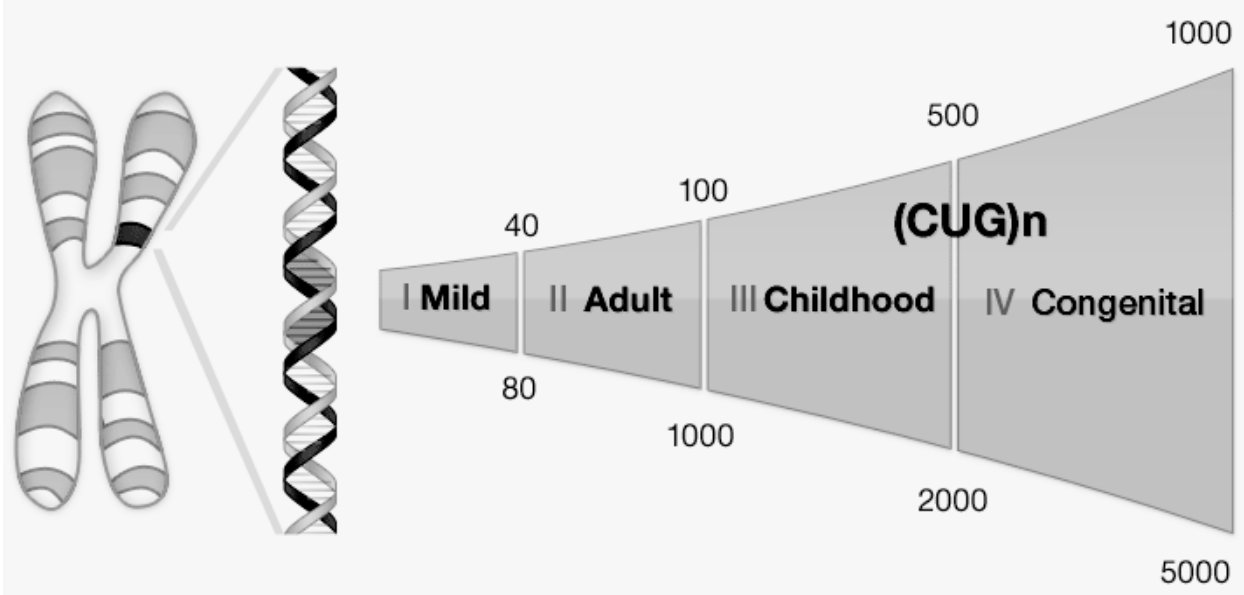

Figure 1.1 The figure shows the CTG repeat length and the overlap between phenotypes (http://myotonicdystrophy.com).

\section{Clinical features}

DM1 patients present a highly variable and multiple systemic manifestation such as cataracts, cardiac disorders, respiratory function abnormalities, and nervous system dysfunction. ${ }^{10}$ Affected individuals often have a characteristic face with facial musculature weakness, ptosis, atrophy of temporal muscles, and premature hair loss. ${ }^{10}$ Reduced initiative, apathy, and avoidant personality have been suggested as cognitive, mental, and personality patterns in DM1. ${ }^{12,13}$ These characteristics can have an impact in clinical settings as they can limit patient involvement in the treatment and may lead to less compliance with therapeutic programs. To the patients, the mental and cognitive characteristics are very debilitating, as they influence working capacity and social interactions.

The main muscular symptoms of DM1 are myotonia (delayed muscle relaxation after contraction) and progressive muscle weakness. Weakness progresses slowly over the years from distal to proximal muscular involvement. Muscular impairment can be assessed clinically using the muscular impairment rating scale (MIRS). ${ }^{14}$ This scale classifies the muscular impairment in five stages: (1) no muscular impairment; (2) minimal signs of muscular impairment; myotonia, jaw and temporal wasting, facial weakness, neck flexor weakness, ptosis, hyper nasal speech, no distal weakness except isolated digit flexor weakness; (3) digital weakness; no proximal weakness except isolated elbow extensor weakness; (4) mild to moderate proximal weakness; (5) severe proximal weakness. Muscle weakness leading to reduced mobility, respiratory problems, and dysphagia are the major causes of disability in the late stages. ${ }^{15}$ DM1 patients usually die from the systemic complications of the disease and pneumonia was 
reported as the most frequent primary cause of mortality followed by death from cardiac causes. ${ }^{16-17}$

\section{Oropharyngeal dysphagia (OD)}

$O D$ is defined as a disorder in the swallowing function. The impairment may involve one or more swallowing stages (oral preparatory, oral, pharyngeal, and/or esophageal) and results from an ineffective transit of the bolus from mouth to esophagus and/or a misdirection of the bolus from the normal swallowing pathway into the airway, resulting in tracheal aspiration. OD has been recognized by the WHO as a medical disability associated with increased morbidity, mortality, and costs of care. ${ }^{18}$ The prevalence of OD in DM1 patients ranges from 25 to $80 \% .{ }^{19-21}$ The high variability in the prevalence of OD presented in the studies may be due to the diversity in the diagnostic tools and methods used to assess swallowing function.

Dysphagia can result from a wide variety of functional or structural deficits of the oral cavity, pharynx, larynx, or esophagus. The main characteristic of OD in DM1 patients is an ineffective propulsion of the bolus in the pharynx and esophagus, resulting in prolonged transit time and bolus residue in the pharynx after swallowing. ${ }^{20,22}$ Studies suggested that swallowing impairment in DM1 is mostly secondary to the weakness of the muscles involved in swallowing function and not to myotonia. ${ }^{20,22}$ OD in this population is more prominent in later stages; however, some studies reported a lack of correlation between disease severity and swallowing dysfunction. ${ }^{20,21,23}$

OD may lead to health complications such as malnutrition and pneumonia. In DM1, swallowing impairment associated with weakness of the diaphragm and respiratory muscles increases the risk of aspiration pneumonia. ${ }^{10,16}$ With longer duration of the disease and progressive muscular weakness, the risk of recurrent pneumonia increases. $^{6}$

Besides the physical consequences, swallowing disorders significantly affect quality of life (QoL) in DM1, as eating and drinking are associated with social and emotional aspects and not only with functional and nutritional aspects. ${ }^{24}$ As a consequence, patients with $\mathrm{OD}$, in addition to experiencing less enjoyment when eating or drinking, may feel embarrassed or isolated in social situations involving eating. Moreover, OD may increase caregiver burden and may require significant lifestyle alterations for the patient and the patient's family. ${ }^{25}$

\section{Evaluation of oropharyngeal dysphagia}

In recent years there has been growing interest in multidimensional assessment for patients with OD. An instrumental evaluation provides information on the pathophysiology of the impairment. But to understand its psychosocial impact, a patient-based, dysphagia-specific measure of QoL is applied, yielding data on how patients experience living with dysphagia. This holistic understanding of OD has therapeutic and management implications. Therefore, in this thesis, we evaluate 
swallowing physiology as well as the patients' perspective of the impact of dysphagia on their QoL to help us understand both dimensions of OD in DM1 patients.

Dysphagia-specific QoL outcomes were assessed using a questionnaire, the SWAL-QOL, which has 44 items and was validated to the Dutch language. ${ }^{26,27}$ It was designed to assess QoL in terms of general burden, food selection, eating duration, eating desire, fear of eating, sleep, fatigue, communication, mental health, social functioning, and symptoms score. Although SWAL-QOL was not designed specifically for the DM1 population, it was validated in a population presenting OD as a result of a variety of neurological diseases and of head and neck cancer.

To assess swallowing physiology, two methods were used in this investigation: videofluoroscopic swallow study (VFS); and fiberoptic endoscopic evaluation of swallowing (FEES). Although these methods are complementary examinations used routinely to confirm clinical suspicion of OD, they differ in technique. VFS is a dynamic $x$-ray examination performed in the radiology department. During the exam the patient sits next to the fluoroscopic tower while the fluoroscopic images capture a lateral view of the oral cavity, pharynx, larynx, and esophagus. At the start of the exam, the patient receives food in different standardized consistencies mixed with barium while the VFS images are captured. The barium makes the food/drink visible during the VFS. The anatomy, structural movements of the tongue, pharynx, larynx, esophagus, and bolus flow characteristics are evaluated and the images are recorded for subsequent analysis. FEES, however, does not require fluoroscopy. The evaluation is performed using a flexible endoscope connected to a computer and video monitor. FEES is usually performed in the outpatient clinic but can also be done at the hospital bedside. The endoscope is inserted through the patient's nose into the pharynx. With the point of the endoscope located above the larynx, the patient is given food in different standardized consistencies colored with methylene blue. The color makes it easier to identify the presence of the food/drink in the pharynx and/or larynx. The images of the pharynx and larynx before and after swallowing are evaluated and recorded for subsequent analysis. During swallowing the constriction of the pharynx blocks the endoscopic view, so the view of the structures is temporarily interrupted (white-out image). In VFS and FEES anatomic and/or physiologic abnormalities are identified relative to the bolus flow and swallowing phase. The findings of the evaluation lead to recommendations regarding the adequacy of the swallow, the advisability of oral feeding, and the use of appropriate interventions to facilitate safe and efficient swallowing. Although VFS and FEES are used for the same purpose, they provide different perspectives on the swallowing process. While VFS is a dynamic radiographic study of the oral, pharyngeal, and esophageal structures, FEES permits an endoscopic view of the pharynx and larynx, including glottal function. The main advantage of VFS in relation to FEES is the integrated observation of all swallowing phases: preparatory, oral, pharyngeal, and esophageal. The disadvantages of VFS consist of exposing the patients to radiation and not being able to perform the examination at the outpatient clinic or as a bedside examination. In 1988, the first article describing the use of FEES on 
swallowing evaluation was published. ${ }^{27}$ Over the years, FEES grew in popularity, as it is a well-tolerated and easy to use instrumental tool to assess swallowing function. ${ }^{28}$ Although FEES is widely used for research purposes and in clinical settings, few studies take an analytical approach to the interpretation of the outcomes of the evaluation. Therefore, two of the studies comprising this thesis were intended to improve our understanding of the FEES scales that were used in our studies to analyze swallow function in DM1 patients. To this end, we investigated two aspects of FEES measurements: (a) the clinimetric features of FEES scales, such as intra- and interobserver agreement; and (b) the extent of within and between subject variability of FEES measurements, once dysphagic patients are subjected to different conditions in routine examinations.

Observers' agreement is the first step to show validity of a measurement. It is not possible to have a valid measurement scale if it is not reproducible in terms of observers' agreement. Lack of reporting observers' agreement compromises the interpretation of the study outcome. Observers' agreement can be influenced by many factors: level of observers' expertise, consistency of the bolus, and well-defined criteria for the variables to be assessed. ${ }^{29-31}$

During a FEES examination patients swallow different consistencies in a pre-determined order. As a result, there is high variability in the swallowing performance between and within patients, depending on the etiology of the swallowing impairment, volume and consistency of the bolus, etc.. ${ }^{32}$ This variability can hinder the identification of normal or impaired swallowing behavior. Moreover, when analyzing swallow trials independently, the examiner might miss the changes and adaptations made by the patients when facing different challenges, such as changes in bolus consistency. Analysis of FEES outcomes with group-based trajectory modeling (GBTM) is described in this thesis. This exploratory approach is used to capture the diversity of the swallowing patterns in dysphagic patients and to identify groups with similar swallowing behavior.

\section{Objectives and outline of the thesis}

Few studies have addressed OD in DM1 and, consequently, the pathophysiology of swallowing in this population remains unclear and warrants further investigation. Over the years, FEES has been used to evaluate swallowing in patients with suspected OD secondary to a variety of diseases such as stroke, Parkinson's, etc. Although FEES is a well-established procedure, its application in the DM1 population is underexplored and the clinimetric characteristics of the measurement scales are not well understood. On these grounds, the studies presented in this thesis were conducted with two objectives. The first was to evaluate and characterize swallowing function and swallow-related QoL in DM1 patients and to determine its association with disease severity. The second was to understand and subsequently improve the clinimetric characteristics of the FEES scales used to assess OD. 
The systematic review presented in chapter 2, was carried out to appraise existing literature on the pathophysiology and diagnostic procedures for OD in DM1. The poor methodological quality of most studies and the limited number of articles on this topic led us to conduct our own investigation of swallowing function in DM1 patients and to include the effects of bolus consistency on swallowing safety. This study, described in chapter 3, was performed by comparing standardized FEES examinations of DM1 patients with those of healthy control subjects. Considering the progressive muscular impairment that characterizes the disease, the association between severity of disease and degree of OD was investigated too. Chapter 4 presents a prospective study designed to investigate DM1 patients' perspective on the impact of swallowing impairment on some aspects of their QoL. A correlation between patients' perception of the impairment collected via a SWAL-QOL questionnaire and the pathophysiological aspects of swallowing assessed using VFS is described there. Chapter 5 reports a study investigating the levels of observers' agreement on FEES measurements in a group of oncological and neurological patients with OD. Visuoperceptual ordinal FEES variables were assessed independently by two observers for this study. It describes aspects influencing the level of agreement, namely, consistency, OD etiology, and rating method. An exploratory study presented in chapter 6 applied an innovative statistical technique, group-based trajectory modeling (GBTM), for the analysis of repeatedmeasures FEES data. Heterogeneity of FEES outcomes makes it difficult for practitioners to pinpoint and characterize regular or irregular patterns of swallowing behavior in population-based studies. By applying this innovative technique, the study could identify subgroups of patients presenting distinctive swallowing performance over the swallow trials, describe the swallowing trajectories' characteristics along seven swallow trials, and associate them with OD etiology, including DM1. The main findings and conclusions of this thesis are presented in chapter $\mathbf{7}$, which also outlines some perspectives for the future. 


\section{References}

1. New nomenclature and DNA testing guidelines for myotonic dystrophy type 1 (DM1). The International Myotonic Dystrophy Consortium (IDMC). Neurology. 2000 Mar 28;54(6):1218-21.

2. Suominen T, Bachinski LL, Auvinen S, Hackman P, Baggerly KA, Angelini C, Peltonen L, Krahe R, Udd B. Population frequency of myotonic dystrophy: higher than expected frequency of myotonic dystrophy type 2 (DM2) mutation in Finland. Eur J Hum Genet. 2011 Jul;19(7):776-82.

3. Medica I, Markovic D, Peterlin B. Genetic epidemiology of myotonic dystrophy in Istria, Croatia. Acta Neurol Scand. 1997;95:164-166.

4. Bouchard G, Roy R, Declos M, Mathieu J, Kouladjian K. Origin and diffusion of the myotonic dystrophy gene in the Saguenay region (Quebec). Can J Neurol Sci. 1989;16:119-22.

5. Deenen JCW, Van Doorn PA, Faber CG, van der Kooi AJ, Kuks JBM, Notermans NC, et al. The epidemiology of neuromuscular disorders: Age at onset and gender in the Netherlands. Neuromuscul Disord. 2016;26(7), 447-52.

6. Harper PS. Myotonic Dystrophy. London, UK: WB Saunders; 2001.

7. Fu YH, Pizzuti A, Fenwick RG Jr, et al. An unstable triplet repeat in a gene related to myotonic muscular dystrophy. Science 1992;255:1256-8. 3.

8. Mahadevan M, Tsilfidis C, Sabourin L, et al. Myotonic dystrophy mutation: an unstable CTG repeat in the 39 untranslated region of the gene. Science 1992;255:1253-5.

9. Brook JD, McCurrach ME, Harley HG, et al. Molecular basis of myotonic dystrophy: expansion of a trinucleotide (CTG) repeat at the 39 end of a transcript encoding a protein kinase family member. Cell 1992;68:799-808

10. Turner C, Hilton-Jones D. The myotonic dystrophies: diagnosis and management. J Neurol Neurosurg Psychiatry. 2010;81(4):358-67

11. Meola G, Cardani R. Myotonic dystrophies: An update on clinical aspects, genetic, pathology, and molecular pathomechanisms. Biochim Biophys Acta. 2015;1852(4):594-606

12. Meola G, Sansone V, Perani D, Scarone S, Cappa S, Dragoni C, Cattaneo E, Cotelli M, Gobbo C, Fazio F, Siciliano G, Mancuso M, Vitelli E, Zhang S, Krahe R, Moxley RT. Executive dysfunction and avoidant personality trait in myotonic dystrophy type 1 (DM-1) and in proximal myotonic myopathy (PROMM/DM-2). Neuromuscul Disord. 2003;13(10):813-21.

13. Meola G., Sansone V. Cerebral involvement in myotonic dystrophies. Muscle Nerve. 2007;36(3): 294-306.

14. Mathieu J, Boivin H, Meunier D, Gaudreault M, Bégin P. Assessment of a disease-specific muscular impairment rating scale in myotonic dystrophy. Neurology. 2001;56(3):336-40.

15. Udd B, Krahe R. The myotonic dystrophies: molecular, clinical, and therapeutic challenges. Lancet Neurol. 2012;11(10):891-905.

16. de Die-Smulders CE, Höweler CJ, Thijs C, Mirandolle JF, Anten HB, Smeets HJ, Chandler KE, Geraedts JP. Age and causes of death in adult-onset myotonic dystrophy. Brain.1998;121(Pt 8):1557-63.

17. Mathieu J, Allard P, Potvin L, Prevost C, Begin P. A 10-year study of mortality in a cohort of patients with myotonic dystrophy. Neurology.1999;52:958-1962.

18. Rommel N, Hamdy S. Oropharyngeal dysphagia: manifestations and diagnosis. Nat Rev Gastroenterol Hepatol. 2016;13:49-59.

19. Bellini M, Biagi S, Stasi C, Costa F, Mumolo MG, Ricchiuti A, Marchi S. Gastrointestinal manifestations in myotonic muscular dystrophy. World J Gastroenterol. 2006; 8;12(12):1821-8.

20. Modolell I, Mearin F, Baudet JS, Gamez J, Cervera C, Malagelada JR Pharyngo-esophageal motility disturbances in patients with myotonic dystrophy. Scand J Gastroenterol. 1999;34(9):878-82.

21. Ertekin C, Yuceyar N, Aydogdu I, Karasoy H. Electrophysiological evaluation of oropharyngeal swallowing in myotonic dystrophy. J Neurol Neurosurg Psychiat. 2001;70(3):363-371

22. Leonard RJ, Kendall KA, Johnson R, McKenzie S. Swallowing in myotonic muscular dystrophy: A videofluoroscopic study. Arch Phys Med Rehabil. 2001;82(7):979-85.

23. Marcon M, Briani C, Ermani M, Menegazzo E, Iurilli V, Feltrin GP, Novelli G, Gennarelli M, Angelini C. Positive correlation of CTG expansion and pharyngoesophageal alterations in myotonic dystrophy patients. Ital J Neurol Sci. 1998;19(2):75-80. 
24. Peric S, Stojanovic VR, Basta I, Peric M, Milicev M, Pavlovic S, Lavrnic D. Influence of multisystemic affection on health-related quality of life in patients with myotonic dystrophy type 1 . Clin Neurol Neurosurg. 2013 Mar;115(3):270-5.

25. American speech-language association (ASHA). Adult Dysphagia. Retrieve in 3 Feb 2017 from http://www.asha.org/Practice-Portal/Clinical-Topics/Adult-Dysphagia/

26. McHorney CA, Robbins J, Lomax K, Rosenbek JC, Chignell K, Kramer AE, Bricker DE. The SWAL-QOL and SWAL-CARE outcomes tool for oropharyngeal dysphagia in adults: III. Documentation of reliability and validity. Dysphagia. 2002 Spring;17(2):97-114.

27. Bogaardt HCA, Speyer R, Baijens LWJ, FokkensWJ. Cross-cultural Adaptation and Validation of the Dutch Version of SWAL-QOL. Dysphagia. 2009;24:66-70.

28. Langmore SE, Schatz K, Olsen N. Fiberoptic endoscopic examination of swallowing safety: a new procedure. Dysphagia 1988;2(4):216-9.

29. Hind JA, Gensler G, Brandt DK, Gardner PJ, Blumenthal L, et al. Comparison of trained clinician ratings with expert ratings of aspiration on videofluoroscopic images from a randomized clinical trial. Dysphagia 2009;24(2):211-7.

30. Scott A, Perry A, Bench J. A study of inter reliability when using videofluoroscopy as an assessment of swallowing. Dysphagia 1998;13(4):223-7.

31. Baijens L, Barikroo A, Pilz W. Intrarater and interrater reliability for measurements in videofluoroscopy of swallowing. Eur J Radiol 2013;82(10):1683-95.

32. Steele CM, Alsanei WA, Ayanikalath S, Barbon CE, Chen J, et al. The Influence of Food Texture and Liquid Consistency Modification on Swallowing Physiology and Function: A Systematic Review. Dysphagia 2015; 30(1):2-26. 


\section{Chapter 2}

\section{Oropharyngeal dysphagia in myotonic dystrophy type 1: a systematic review}

Walmari Pilz

Laura W.J. Baijens

Bernd Kremer

Dysphagia. 2014;29(3):319-31 


\section{Abstract}

A systematic review was conducted to investigate the pathophysiology of and diagnostic procedures for oropharyngeal dysphagia in myotonic dystrophy (MD). The electronic databases Embase, PubMed, and The Cochrane Library were used. The search was limited to English, Dutch, French, German, Spanish, and Portuguese publications. Sixteen studies met the inclusion criteria. Two independent reviewers assessed the methodological quality of the included articles. Swallowing assessment tools, the corresponding protocols, studies' outcome measurements and main findings are summarized and presented. The body of literature on pathophysiology of swallowing in dysphagic patients with MD type 1 remains scant. The included studies are heterogeneous with respect to designs and outcome measures and hence not directly comparable. More importantly, most studies had methodological problems. These are discussed in details and recommendations for further research on diagnostic examinations for swallowing disorders in patients with myotonic dystrophy type 1 are provided. 


\section{Introduction}

Myotonic Dystrophy (MD) type 1, also known as Steinert's disease, is an autosomal dominant disorder. It is the most common form of adult-onset muscular dystrophy, with prevalence reported to range from 2.1 to 14.3 per 100,000 worldwide. ${ }^{1}$ MD is a multisystem disorder that affects visual, endocrine, cardiac, and central nervous systems. The clinical findings can include ptosis, weakness of the facial, jaw, and anterior neck muscles, cataract, conduction cardiac defects, intellectual and learning disabilities, and gastrointestinal disturbances which may result in malnutrition and weight loss. ${ }^{2}$ Muscle weakness and myotonia characterized by sustained involuntary muscle contractions are the main symptoms. Distal muscles are the first to be affected. The progression of the muscular weakness is usually slow, but MD can be severely disabling when proximal muscles weakness develops, affecting daily activities and quality of life. ${ }^{3}$

Oropharyngeal dysphagia is a common symptom among the MD subjects and can lead to a variety of medical complications such as respiratory and nutritional problems. In the MD population, the association of swallowing and respiratory muscles weakness can increase the frequency of aspiration and pneumonia. ${ }^{4}$ Pneumonia and respiratory problems have been shown to be the most frequent cause of death, ranging from 31 to 43\%. ${ }^{4,5}$ Videofluoroscopy (VFS) and fiberoptic endoscopic evaluation of swallowing (FEES) are the recommended instrumental tools for assessing swallowing. ${ }^{6}$ Currently, there are no disease-specific guidelines to assess or manage swallowing problems for the MD population. ${ }^{7}$

Although a high incidence of dysphagia among MD patients is reported, the pathophysiological mechanisms of oropharyngeal dysphagia remain unclear. ${ }^{8} \mathrm{~A}$ few reviews have been performed on this topic but they were not conducted by applying a systematic search protocol and no qualitative analyses of the included studies were performed. The aim of this systematic review was to investigate the pathophysiology of and diagnostic procedures for oropharyngeal dysphagia in MD and to conduct a qualitative analysis of the included studies.

\section{Methods}

\section{Identification and Selection of Studies}

A search of the electronic biomedical databases Embase, PubMed, and The Cochrane Library was performed. The search was limited to article published in the English, German, French, Spanish, Portuguese, and Dutch. In Embase, Thesaurus terms were combined as presented in Table 2.1. All trees of subject headings were explored. The search was expanded by using free-text words (truncation or wildcards). In PubMed and in The Cochrane Library database, Medical Subject Heading (MeSH) terms were 
recognized and all trees of these MeSH terms were explored and combined in the biomedical literature database (Table 2.1). The free-text words used in Embase were explored in PubMed and in The Cochrane Library database (Table 2.1).

Two reviewers independently assessed each abstract for potential inclusion. Full-text articles of all the selected abstracts were retrieved and reviewed to make a definitive decision regarding inclusion. In the event that the two reviewers did not agree on inclusion, consensus agreement was made after applying the inclusion criteria to the full text version of said article. The reference lists of all included articles were searched for additional literature. All studies that examined the swallowing mechanism of adults suffering from MD type 1 were included, without restrictions regarding disease duration or severity. Excluded were conference papers, review papers, letters to the editor, experiments on animals, articles published before 1980, studies describing pathophysiology of esophageal dysphagia without information on oropharyngeal deglutition, studies presenting a heterogeneous subject population with different subtypes of myopathies or other neurological diseases that did not described the outcome for the MD subgroup. Studies with sample-sizes below five were excluded as well.

Table 2.1 Systematic syntax of the search in the three biomedical literature databases

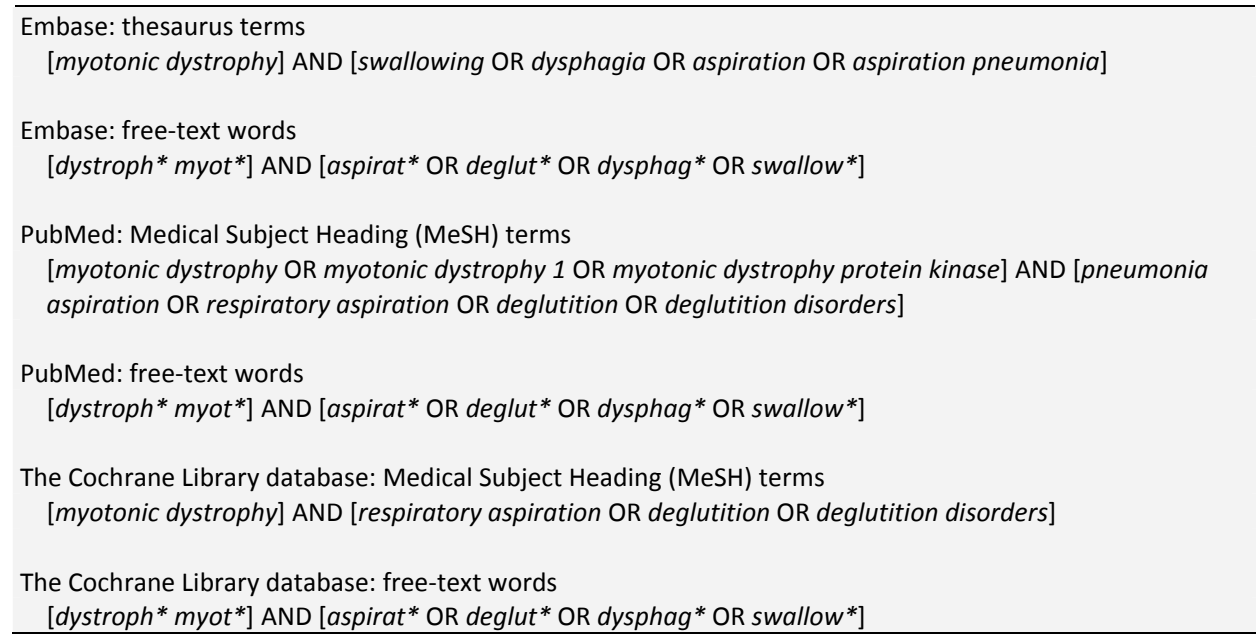

\section{Data analysis and assessment of study quality}

The aim of a study quality assessment is to provide guidelines for the interpretation of findings and aid in determining the strength of the evidences of the included studies. ${ }^{9}$ Therefore, the methodological quality of the included articles was evaluated using a 10item critical appraisal derived from The Cochrane Collaboration 2009 and the Quality Assessment of Diagnostic Accuracy Studies (QUADAS) tool. ${ }^{9,10}$ The present assessment of study quality consists of ten criteria presented in Table 2.2. Criteria were scored 
"yes", "no" or "unclear" when information was not available or not clearly described. When a specific criterion could not occur due to its absence, it was scored as "not applicable (NA)". External validity (generalizability) was assessed with criteria 1, 2, 5 and 6, internal validity with criteria 3, 4, 7, 8, and 9 and statistical methods with criterion 10. After training, two reviewers independently assessed the study quality of the included articles without being blinded to the journal and the authors. There were few differences in their scoring and these were settled by discussion.

Table 2.2 Critical appraisal criteria for methodological quality assessment

\begin{tabular}{ll}
\hline Item & Description \\
\hline 1 & Was the spectrum of patients representative of the MD patients in clinical practice? \\
3 & Were selection criteria clearly described? (inclusion and exclusion criteria) \\
& Was the time period between different swallowing assessments short enough to be reasonably sure \\
4 & Did all patients undergo the same standardized assessment protocol? \\
5 & Was the execution of the assessment described in sufficient detail to permit replication of the test? \\
6 & Were the outcome measurements described in sufficient detail to permit replication of the study? \\
7 & Were the raters blinded to the group and to patients' medical history? \\
8 & Were the raters blinded to each other's results? \\
9 & Were withdrawals (drop-outs) from the study explained? \\
10 & Method of data analyses. Were appropriate statistics methods applied?
\end{tabular}

\section{Results}

\section{General results}

A total of 409 articles were found. Of these, 76 articles were selected in PubMed using MeSH terms, 77 articles were found in Embase using Thesaurus terms and no articles were found in The Cochrane Library. The search using free-text words revealed 157 articles in PubMed, 97 articles in Embase, and 2 articles in The Cochrane Library. Of the 409 titles and abstracts of articles reviewed, 380 were excluded because they did not meet the inclusion criteria. After conducting a full-text review of the remaining 29 articles, 16 were selected for further analysis based on the inclusion criteria. Additional studies were not found in the reference lists of the included articles. Of the final 16 articles, 9 studies were case-control, ${ }^{11-19} 6$ were case-series, ${ }^{20-25}$ and 1 was a crosssectional survey. ${ }^{26}$ Descriptive statistics were used in four studies. ${ }^{21,23-25}$ The majority of the studies applied inferential statistical procedures to evaluate swallowing outcome in the MD group. ${ }^{11-20,22,26}$ An overview of the 16 included articles is presented in Table 2.3. 


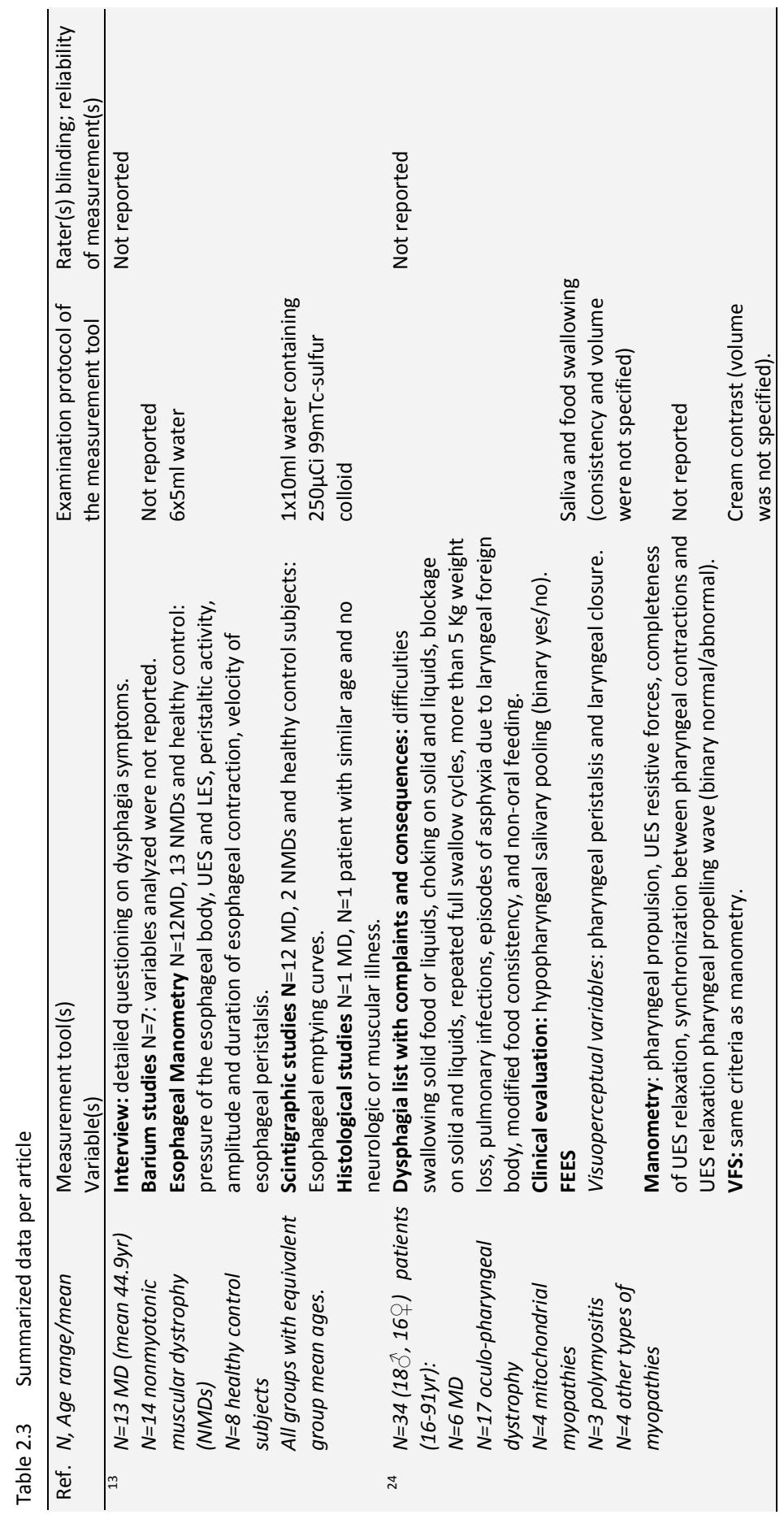




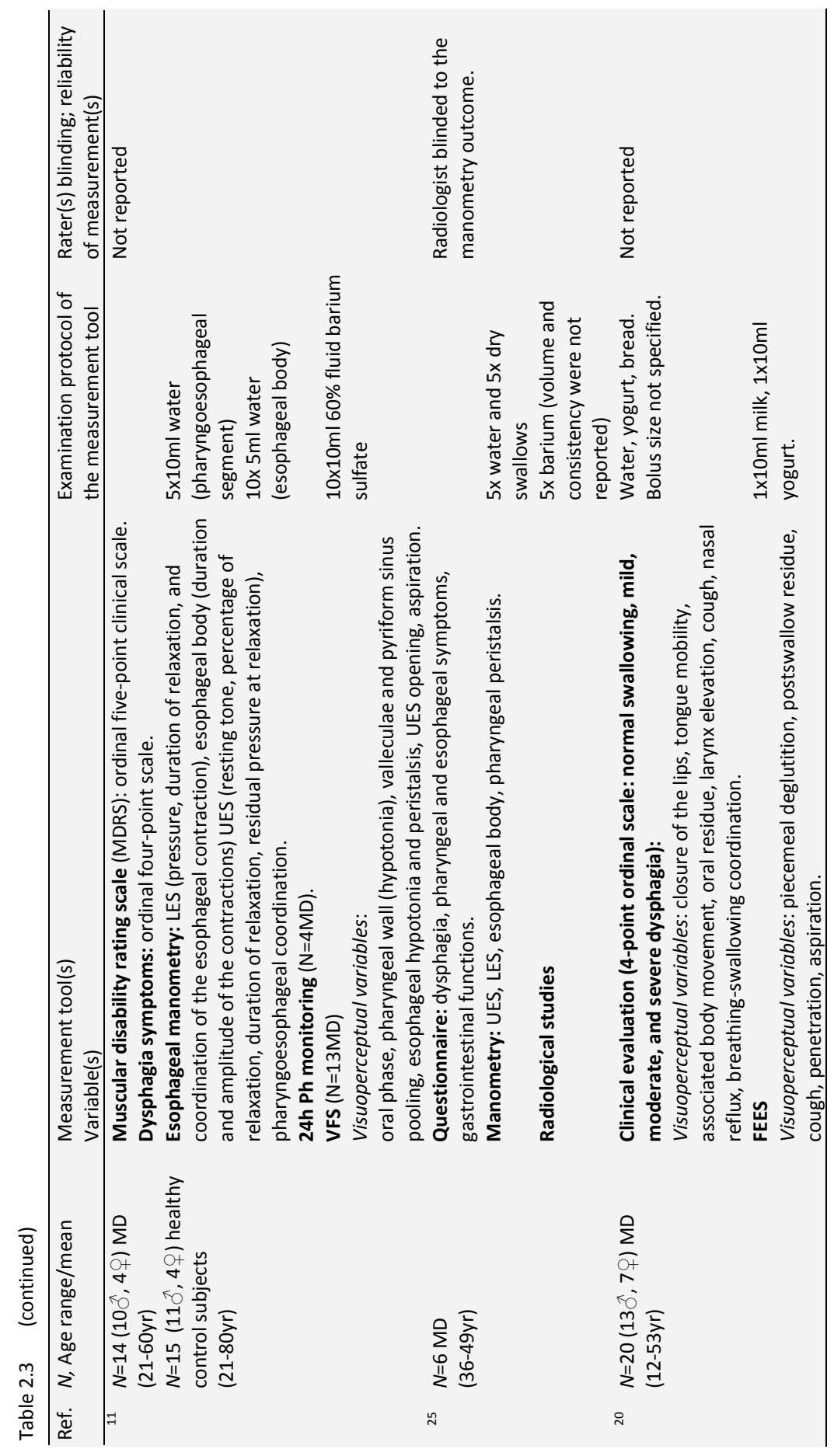




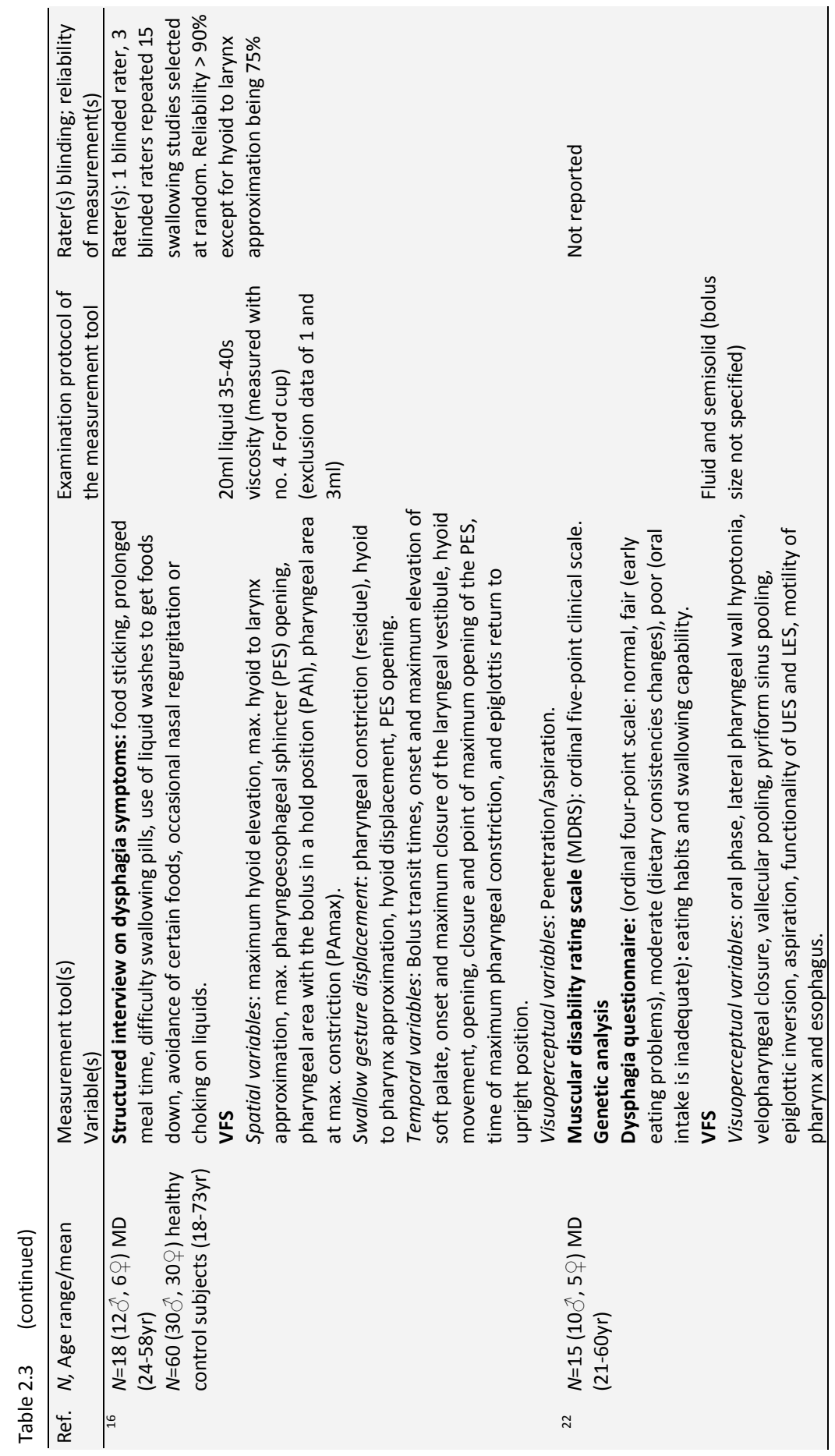




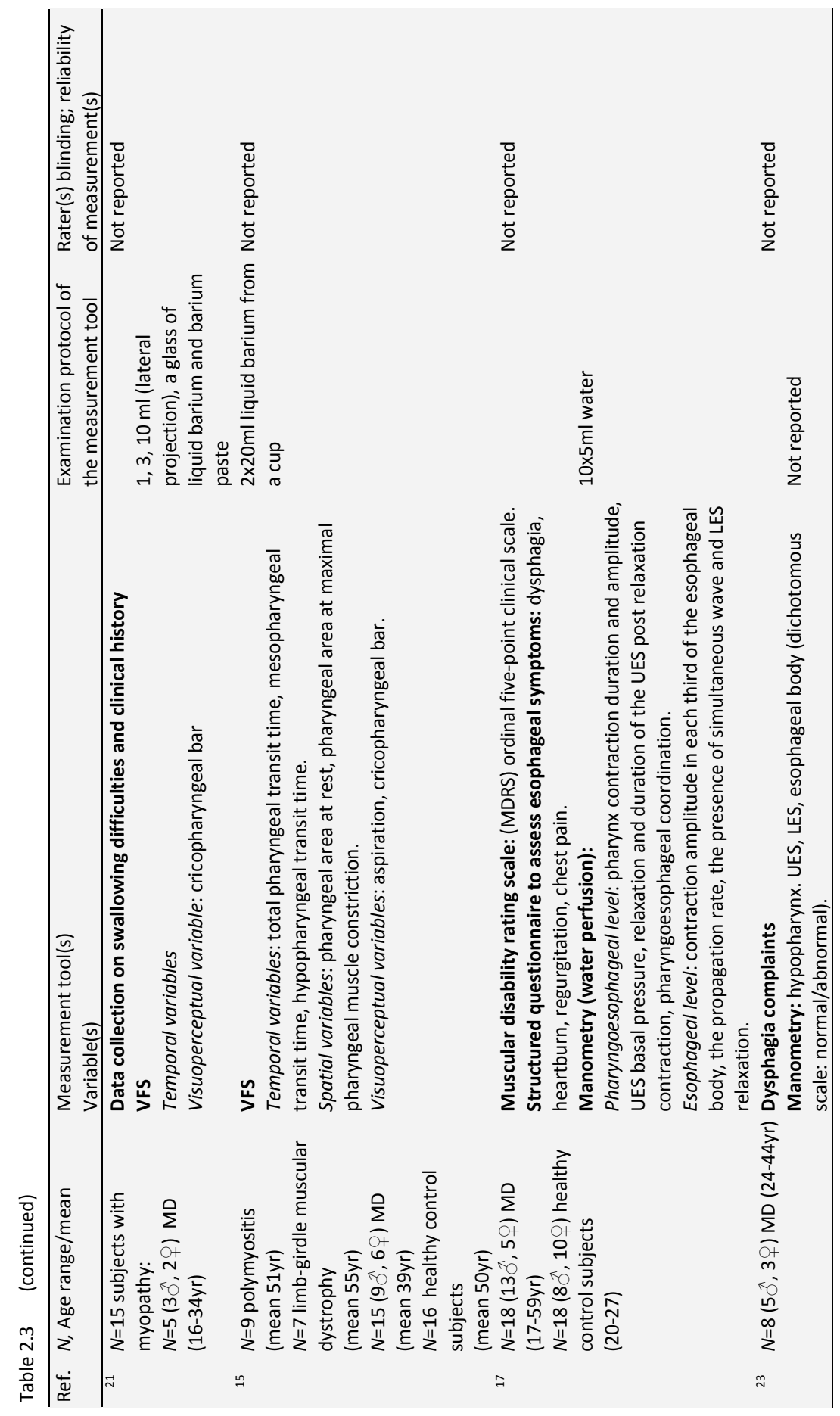




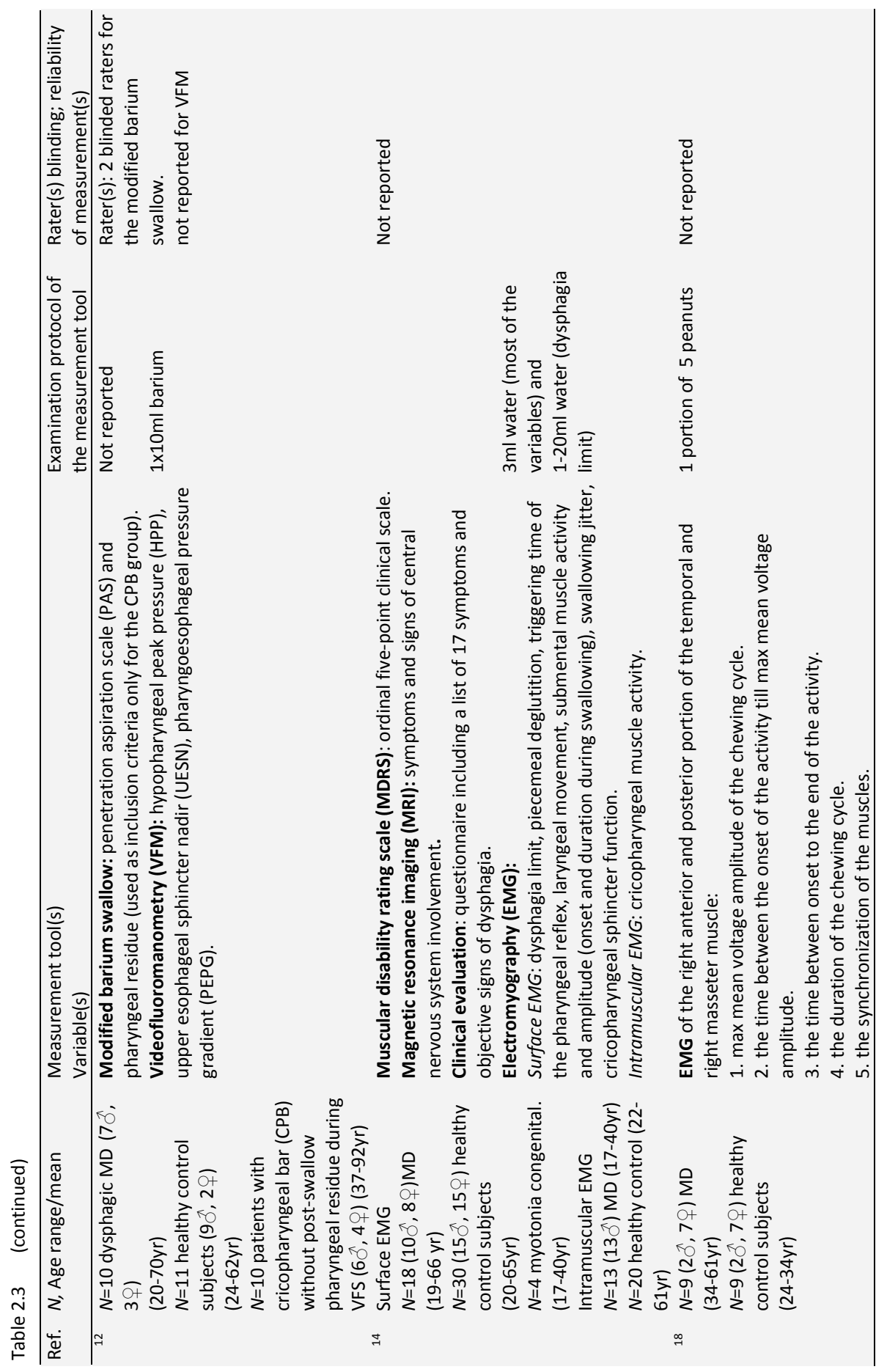




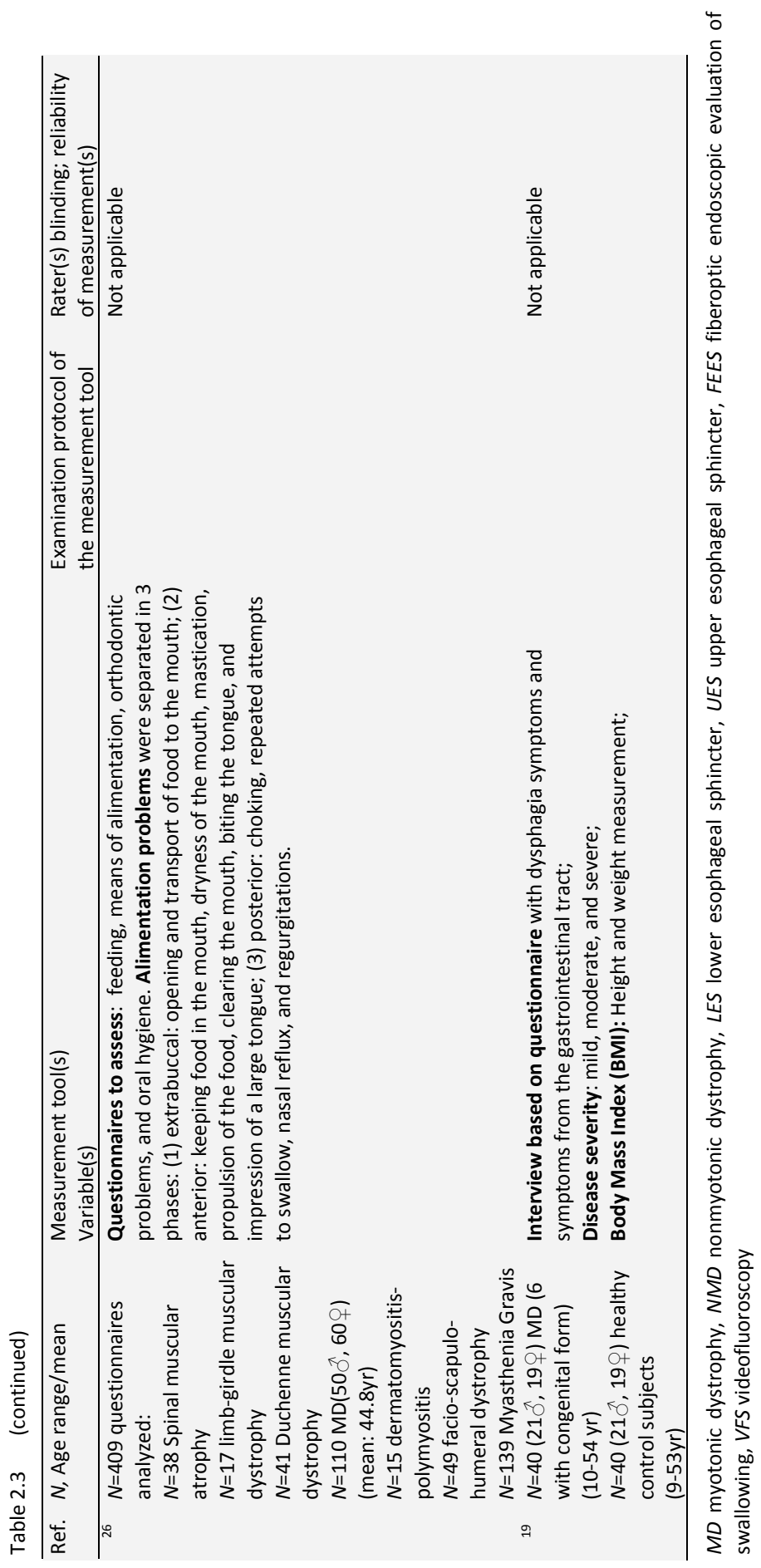


Meta-analysis could not be performed because of the heterogeneity in study designs and lack of standardization in swallowing assessment. The included articles are ordered according to the number of applied swallowing assessment tools used, with the study that used the largest number of tools listed first. Sample size, characteristics of the population (age and gender), measurement tool, variables analyzed, protocol, and measurement reliability if present are summarized (Table 2.3).

\section{Quality of studies}

The methodological quality of the included studies is presented in Table 2.4. One of the studies fulfilled all four criteria for external validity ${ }^{14}$ and 3 studies fulfilled three criteria. $^{12,19,20}$ One study fulfilled four of the five criteria for internal validity representing a low risk of bias. ${ }^{16}$ The statistical methods applied appeared to be adequate and described in sufficient detail in eight studies. For five studies item 10 was scored as unclear since the applied statistical methods were not reported in sufficient detail to allow evaluation. For one of the 4 studies using descriptive statistics, item 10 was scored "no" as the results of the swallowing assessment of one MD patient were not reported. ${ }^{21}$

Table $2.4 \quad$ Methodological quality assessment of the included studies

\begin{tabular}{|c|c|c|c|c|c|c|c|c|c|c|}
\hline Included studies & Item 1 & Item 2 & Item 3 & Item 4 & Item 5 & Item 6 & Item 7 & Item 8 & Item 9 & Item 10 \\
\hline [11] & $\mathrm{N}$ & $U$ & $U$ & $\mathrm{~N}$ & $Y$ & $\mathrm{~N}$ & $U$ & $U$ & $Y$ & $U$ \\
\hline [12] & $U$ & $Y$ & NA & $Y$ & $Y$ & $Y$ & $U$ & $U$ & $Y$ & $Y$ \\
\hline [13] & $U$ & $Y$ & $U$ & $\mathrm{~N}$ & $\mathrm{~N}$ & $\mathrm{~N}$ & $U$ & U & $\mathrm{N}$ & $U$ \\
\hline [14] & $Y$ & $Y$ & $U$ & $\mathrm{~N}$ & Y & $Y$ & $U$ & $U$ & $Y$ & $U$ \\
\hline [15] & $\mathrm{U}$ & $\mathrm{N}$ & NA & $Y$ & $\mathrm{Y}$ & $\mathrm{Y}$ & U & U & $Y$ & Y \\
\hline [16] & $U$ & $\mathrm{~N}$ & $U$ & $Y$ & $Y$ & $\mathrm{Y}$ & $\mathrm{Y}$ & $\mathrm{Y}$ & Y & $U$ \\
\hline [17] & $U$ & $U$ & $U$ & $Y$ & $U$ & $\mathrm{Y}$ & $U$ & U & $\mathrm{Y}$ & $Y$ \\
\hline [18] & $U$ & $\mathrm{~N}$ & NA & $Y$ & $\mathrm{Y}$ & $\mathrm{Y}$ & $U$ & $U$ & $Y$ & $Y$ \\
\hline [19] & $Y$ & $Y$ & NA & $Y$ & $Y$ & $\mathrm{~N}$ & $U$ & NA & $Y$ & $Y$ \\
\hline [20] & $U$ & $Y$ & $Y$ & $Y$ & $Y$ & $Y$ & $U$ & U & $Y$ & $Y$ \\
\hline [21] & U & $\mathrm{N}$ & $U$ & $Y$ & $\mathrm{~N}$ & $Y$ & U & U & $Y$ & $\mathrm{~N}$ \\
\hline [22] & $Y$ & $\mathrm{U}$ & $U$ & $Y$ & $\mathrm{~N}$ & $Y$ & $U$ & $U$ & $Y$ & $Y$ \\
\hline [23] & $U$ & $\mathrm{~N}$ & $U$ & $Y$ & $\mathrm{~N}$ & $Y$ & $U$ & U & $Y$ & NA \\
\hline [24] & $U$ & $\mathrm{~N}$ & $U$ & $N$ & $\mathrm{~N}$ & $N$ & $U$ & U & $Y$ & NA \\
\hline [25] & $U$ & $\mathrm{~N}$ & $Y$ & $Y$ & $\mathrm{~N}$ & $\mathrm{~N}$ & $U$ & $U$ & $Y$ & $U$ \\
\hline [26] & $U$ & $U$ & NA & $Y$ & $Y$ & $Y$ & NA & NA & $U$ & NA \\
\hline
\end{tabular}

$Y$ yes (met criteria), $N$ no (not meet criteria, $U$ unknown because insufficient information provided, $N A$ not applicable

\section{Description of swallowing assessment tools and findings}

Various methods were applied to assess swallowing function across these studies. Even though some studies used the same instrumental tool, the applied protocols and the 
way the variables were operationalized varied. In the following subsections studies are summarized according to the number of applied swallowing assessment tools. The study using the largest number of tools is described first.

\section{Dysphagia symptoms, Barium study, Manometry, and Scintigraphy}

Eckardt et al. ${ }^{13}$ designed a study to evaluate esophageal motor function in MD. Dysphagia symptoms were collected via interview. Seven of the 13 MD patients who reported dysphagia underwent barium studies of the esophagus. The exams were interpreted as being normal. Manometry showed a posterior upper esophageal sphincter (UES) resting pressure markedly reduced in MD patients compared to that in healthy subjects; however, the duration of sphincter relaxation was similar in both groups. The data of the scintigraphic studies confirmed the presence of an esophageal motility disturbance in these patients. The pathological findings from the histological examination of the esophagus revealed abnormalities that were confined to the striated muscle fibers. The authors concluded that the main characteristic of the esophageal motility disturbance in MD patients is muscular weakness of the UES and the esophageal body.

\section{Structured patient interview, FEES, Manometry, and VFS}

In the study by St Guily et al., ${ }^{24}$ patients with different muscular diseases were evaluated to identify clinical and functional consequences of muscular involvement and discuss therapeutic indications for dysphagia. All six MD patients complained of choking and five reported blockage during solid food intake. Nasal regurgitation and piecemeal deglutition were also frequent complaints. Two MD patients reported weight loss of more than $5 \mathrm{~kg}$ and pulmonary infection due to aspiration. The results of the swallowing assessment with FEES, manometry, and VFS were summarized in two outcome variables: pharyngeal propulsion (including aperistalsis) and function of the UES. There was a decrease in pharyngeal propulsion in two MD patients during manometry and VFS and in one patient during FEES. A disturbed UES function was found in three MD patients during manometry and in one patient during VFS. Although three different parameters of the UES function were measured in the study (Table 2.3), a detailed description of them was not reported. The authors' conclusions were based on the findings of all the muscular diseases included in the study, without any specific conclusions for the MD group.

\section{Questionnaire on Dysphagia Symptoms or Clinical History of Dysphagia, Manometry, and VFS}

Constantini et al. ${ }^{11}$ investigated the pharyngoesophageal motor pattern in MD patients. Dysphagia, ranging from mild to severe, was reported by six patients. The results of the manometric examination showed a reduced UES resting tone with normal duration of 
the relaxation, normal residual pressure at relaxation, and pharyngoesophageal coordination. The contraction amplitude in the proximal pharynx was lower in the MD group than in the healthy group. Moreover, lower amplitudes in pharyngeal contraction were observed in the group with dysphagia complaints than in MD patients without complaints. The results of the VFS examination showed that pooling of contrast in the pharynx was observed in 12 of the 14 patients. Aspiration was observed in one patient. According to the authors, the current findings are consistent with and similar to those of previous reports which confirmed that MD patients present a typical pharyngeal and esophageal motor pattern characterized by reduced pharyngeal contraction and reduced UES tonic resting pressure. Moreover, pharyngoesophageal myotonia was rejected as a disease-specific feature since no groups differences (patients versus healthy controls) in the duration of the pharyngoesophageal contraction were found. In the study by Swick et al., ${ }^{25}$ pharyngoesophageal motor function in MD patients was investigated. Two of the six patients reported swallowing difficulties. Low amplitudes and normal durations of the pharyngeal peristaltic wave were observed in all six MD patients. UES relaxed normally, but the resting pressure in the UES was decreased in five patients. VFS data confirmed these manometric findings, showing an intact but weak pharyngeal peristalsis in all patients. The authors concluded that pharyngoesophageal dysfunction was common in $\mathrm{MD}$, even when clinical symptoms were absent. Moreover, the abnormalities found might be due to muscle weakness rather than myotonia.

\section{Clinical evaluation of swallowing and FEES}

The swallowing mechanism of MD patients was studied by Chiappetta et al. ${ }^{20}$ to evaluate and classify oropharyngeal dysphagia. During clinical evaluation 1 of the of the 20 patients (5\%) was classified as having a normal swallowing function. Twenty percent of the patients presented severe dysphagia, 30\% moderate, and $45 \%$ mild dysphagia. FEES results showed that pharyngeal peristalsis was decreased in $70 \%$ of the patients using thick-liquid consistency and in $60 \%$ of the patients using thin-liquid consistency. Aspiration was observed in $20 \%$ of the patients. When comparing swallowing performance relative to the consistency of the food, MD patients presented more abnormalities when swallowing thick-liquid consistency.

\section{VFS}

Among the included articles, four studies used VFS to identify and describe the characteristics of dysphagia in MD patients. Leonard et al. ${ }^{16}$ compared the swallowing function in MD patients to that of healthy subjects, identifying the contributors to and predictors of improvement, and evaluating strategies that facilitate swallowing. All 18 MD patients reported some level of swallowing difficulty during a detailed interview. VFS analysis revealed several differences between MD patients and the healthy controls. The MD group exhibited increased oropharyngeal and hypopharyngeal transit 
times. The onset of soft palate elevation, aryepiglottic fold closure, and hyoid displacement was delayed and the return of the epiglottis to the upright position was slower. A reduced hyoid displacement was observed only in male MD patients. Moreover, the pharyngeal area at maximum constriction was larger in MD patients than in healthy controls, and pharyngeal residue was observed as a consequence of the incomplete pharyngeal constriction. It was also observed that the pharyngoesophageal segment (PES) opened early, relative to the arrival of the bolus at the PES, and was prolonged in MD subjects. Three patients aspirated. The authors concluded that the most significant difference between the groups was the presence of pharyngeal residue in the MD group caused by an ineffective pharyngeal constriction.

Marcon et al. ${ }^{22}$ investigated a possible pattern in the swallowing function of $\mathrm{MD}$ patients and its correlation with the size of CTG repeats. Seven patients were reported as having a degree of dysphagia ranging from mild to poor. VFS findings indicated that there were only three subjects without dysphagia. Pharyngeal residue was present in six and silent aspiration occurred in three patients. Incomplete UES relaxation and hypotonic esophagus were the most common alterations of swallowing in MD. The authors concluded that an atonic pharynx may be a specific characteristic of MD patients and recommended that VFS be part of the evaluation of all MD patients.

The study by Johnson and McKenzie et al. ${ }^{21}$ demonstrated the use of quantitative kinematic pharyngeal transit time in the evaluation of patients with myopathy. All five patients had swallowing complaints and three of them reported a history of respiratory infection. A prolongation in the hypopharyngeal transit time and a normal mesopharyngeal transit time were observed in the VFS of the MD patients. Pooling in the valleculae or the pyriform sinus was present in all exams. The authors reported that kinematic pharyngeal transit time may not completely reflect the clinical severity of dysphagia in MD.

In a later study, Johnson et al. ${ }^{15}$ evaluated patients with three types of myopathy to determine the specific swallowing characteristics per myopathy. Results of the VFS showed a significant difference in total pharyngeal transit time between the control group and the groups with myopathy. The percentage of pharyngeal area remaining unclosed during maximal pharyngeal constriction was higher in the MD group. Aspiration was observed in $13 \%$ of the MD patients. Considering the severe impairment of pharyngeal constriction in $\mathrm{MD}$, the incidence of aspiration was low and may suggest an adaptation or accommodation to the disease.

\section{Manometry}

The aim of the study by Modolell et al. ${ }^{17}$ was to characterize pharyngoesophageal motor abnormalities in MD patients and investigate the correlation between these abnormalities, clinical manifestation, and peripheral neuromuscular involvement. Seven of the 18 patients reported no complaints of pharyngoesophageal dysphagia. Manometry results of MD patients relative to the control group showed lower 
amplitude in the pharyngeal contraction while the mean duration of the contraction did not differ, and a lower UES resting pressure with a similar duration of the UES relaxation during swallowing. The esophageal contraction amplitude was diminished in all patients. In conclusion, the authors confirmed the existence of abnormalities in the motility of the pharynx, UES, and especially in the esophageal body of MD patients.

Ramirez et al. ${ }^{23}$ investigated esophageal motor function in MD. Four of the eight patients presented dysphagia symptoms. All but one patient presented diminished amplitude of the contraction in the hypopharynx. The UES presented a diminished tonus in five patients. According to the authors, the results suggested that striated and smooth muscle are impaired in MD patients.

\section{Videofluoromanometry}

Desuter et al. ${ }^{12}$ used videofluoromanometry (VFM) to assess three swallowing physiological variables. There were significant group differences in hypopharyngeal peak pressure (HPP) and pharyngoesophageal pressure gradient (PEPG). The MD group had the lowest values for both parameters (below $100 \mathrm{mmHg}$ ). No group difference was found for upper esophageal sphincter nadir (UESN). The authors concluded that the use of HPP or PEPG as physiological variables provided quantitative data that allow VFM to discriminate distinctive swallowing conditions in the three groups studied.

\section{Electromyography (EMG)}

Two studies evaluated swallowing function using electromyography (EMG). The aim of the study by Ertekin et al. ${ }^{14}$ was to show the existence and frequency of subclinical electrophysiological abnormalities in oropharyngeal swallowing and to clarify the mechanisms of dysphagia in MD. The results showed that the MD group presented (1) piecemeal deglutition (67\%) as a result of weakness of the lingual, masticatory, buccal, and submental muscles; (2) delayed triggering of the pharyngeal swallowing reflex; (3) increased duration of the swallowing reflex indicating a slow pharyngeal phase; (4) increased swallowing jitter that may result from weakness of the laryngeal elevators; and (5) an intact UES muscle in most MD patients. The authors concluded that both myopathic weakness and myotonia encountered in oropharyngeal muscles play an important role in the oral and the pharyngeal phases of swallowing dysfunction in MD. EMG activity of the masticatory muscles was analyzed in the study by Odman and Kiliaridis. ${ }^{18}$ The findings showed that the MD group had reduced masticatory muscle activity during maximal clenching and chewing when compared with healthy subjects. Group differences in resting position or in the speed of chewing were not found. The MD group required more chewing cycles to triturate the food. There was great individual variation in the MD group which may have been due to the diversity in the group in regard to onset and severity of the muscle involvement. 


\section{Structured questionnaires}

Two articles provided information about symptoms and complaints of dysphagia using structured questionnaires. Willig et al. $^{26}$ conducted a survey including several neuromuscular diseases. Statistical analyses showed that choking, repeated attempts to swallow and regurgitation were the main complaints in the MD population. Choking was the most frequent complaint. Even though there was a high prevalence of abnormalities in the swallowing function in this population, only a few patients reported changes in food consistency.

In the study by Ronnblom et al. ${ }^{19}$ the authors investigated the frequency of the gastrointestinal symptoms in $40 \mathrm{MD}$ patients and their relationship with duration and severity of the disease. Moreover, the impact of the disease on nutritional state was also analyzed. No statistically significant difference was found in body mass index (BMI) between MD and healthy control subjects. Dysphagia and coughing while eating were present in almost half of the patients. The authors concluded that many patients with MD develop gastrointestinal symptoms a long time before the more classical muscular symptoms occur.

\section{Discussion}

\section{Methodological comment}

In the present systematic review, the majority of the included studies were limited in one way or another in regard to methodological quality. The sample sizes of the MD group were generally small, thus precluding generalization of these results to all patients with MD. ${ }^{12,18,21,23,25}$ Only five studies reported MD disease severity. ${ }^{11,14,17,19,22}$ Disease duration or time since diagnosis was not always reported. . $^{13,15,17,19,21,24,26}$ Considering the fact that MD is a progressive neuromuscular disease, data regarding severity and duration of the disease cannot be ignored. Furthermore, the distribution of the disease severity and duration of the examined MD population can affect the diagnostic outcome and the effect of therapy. Nine studies ${ }^{11-19}$ compared the results of the MD group with a healthy control group although only three of them were matched for age. ${ }^{11,14,19}$ For several studies it was not clear if a statistical test was used to examine age differences.

One study used reference values for kinematic transit time that were derived from a prior study of stroke patients. ${ }^{21}$ It can be argued that it is methodologically unsound to take a group with a totally different underlying disease as a reference.

Several studies lacked a clear documentation of the clinical assessment of dysphagia complaints and symptoms. Although some patients had a clinical diagnosis of dysphagia, no information was provided as to how the data were gathered. ${ }^{11,23}$ In two studies, questionnaires were the only tool applied to investigate alimentation problems $^{26}$ and gastrointestinal symptoms (including swallowing difficulties). ${ }^{19}$ It was 
unclear whether the psychometric instrument applied had been validated in a dysphagic population. Absence of subjective swallowing complaints in MD patients even in the presence of significant abnormalities during instrumental examination was often reported. ${ }^{13,16,17,22,25}$ Therefore, a multidimensional swallowing evaluation including complimentary assessment tools is necessary to detect the presence of dysphagia. Although some studies used more than one instrument to assess swallowing, not all the subjects underwent the same examinations. The inclusion and exclusion criteria for a particular exam were not described. ${ }^{11,24,25}$ Underreporting of selection criteria introduces potential bias. ${ }^{9}$

Most studies clearly described the applied instrumental assessment tools for dysphagia. However two studies did not report the VFS outcome variables used to assess swallowing. ${ }^{13,25}$ Although several outcome variables were evaluated by St. Guily et al., ${ }^{24}$ results on these variables were very limited. In three studies more than one bolus consistency was used to evaluate swallowing. ${ }^{20-22}$ However, only one presented the results of the swallowing performance according to the consistency tested. ${ }^{20}$ Lack of detailed information regarding the protocol applied for the swallowing examination precludes replication of the study. Differences in examination procedure present potential sources of diagnostic variation and bias. ${ }^{10}$ Information about the number of observers used to evaluate the exams were absent in almost all the studies. Only one study reported data on interrater reliability. ${ }^{16}$ Any swallowing study should provide information on the training protocol of raters and the intrarater and interrater reliability to facilitate interpretation of the study outcome.

\section{Limitations}

The present systematic review has limitations with respect to the search strategy, quality assessment, and data analysis. The systematic search conducted to perform this review generated a low number of articles. A reason for this may be the inconsistent terminology used in studies on oropharyngeal dysphagia and MD. Furthermore, the search strategy may have been too specific or the number of applied biomedical literature databases too limited. However, bibliographic control issues of grey literature and the unsuccessful and labor-intensive attempt to contact authors resulted in the decision to use the three most important biomedical databases relevant for clinicians. Next, the assessment of study quality was performed using critical appraisal items derived from the Cochrane Collaboration $2009^{9}$ and the QUADAS tool. ${ }^{10}$ Another method of methodological quality assessment may have given different results.

\section{Implication for practice}

Although no generalized meta-analytic conclusions can be drawn from the pool of included articles, dysphagia is clearly a common symptom in MD patients. Pharyngeal pooling ${ }^{11,12,15,16,20-22,24,25}$ possibly associated with a decreased pharyngeal contraction amplitude, $^{11,13,17,23,25}$ and reduced UES resting pressure ${ }^{11,13,17,23,25}$ were the main 
findings. Specific trends in the diagnostic or clinical identification of dysphagia in MD were observed. Considering the severity and the medical costs of dysphagia complications, particularly pneumonia, a multidimensional swallowing assessment should be conducted in all MD patients.

\section{Recommendation}

There is need for well-designed research to investigate the pathophysiology of swallowing in MD patients. Future research on pathophysiological aspects of swallowing in MD may include study designs with larger numbers of dysphagic patients and healthy control subjects. It would be interesting to include patients with different stages of disease severity and/or disease duration to understand differences in swallowing physiology. Multidimensional dysphagia assessment needs to contain welldefined protocols during instrumental measurements, analysis of intrarater and interrater reliability, training protocol for the expert raters, and blinding of the raters to group. 


\section{References}

1. Harper, PS. Myotonic Dystrophy. London: Saunders;2001

2. Turner C, Hilton-Jones D. The myotonic dystrophies: diagnosis and management. J Neurol Neurosurg Psychiatry. 2010;81(4):358-67.

3. Natterlund B, Gunnarsson LG, Ahlstrom G. Disability, coping and quality of life in individuals with muscular dystrophy: a prospective study over five years. Disabil Rehabil. 2000;22:776-785

4. Mathieu J, Allard P, Potvin L, Prévost C, Bégin P. A 10-year study of mortality in a cohort of patients with myotonic dystrophy. Neurology. 1999;52(8):1658-1662.

5. de Die-Smulders CE, Höweler CJ, Thijs C, Mirandolle JF, Anten HB, Smeets HJ, Chandler KE, Geraedts JP. Age and causes of death in adult-onset myotonic dystrophy. Brain.1998;121(Pt 8):1557-63.

6. Taylor-Goh, S.Royal College of Speech \& Language Therapists (RCSLT) Clinical Guidelines: 5.8 Disorders of Feeding, Eating, Drinking \& Swallowing (Dysphagia), 2005. http://www.asha.org/members/ guidelines.aspx?id=6901. Acessed 5 July 2012.

7. LaDonna KA, Koopman WJ, Venance SL. Myotonic Dystrophy (DM1) and dysphagia: the need for dysphagia management guidelines and an assessment tool. Can J Neurosci Nurs. 2011;33(1):42-6.

8. Bellini M, Biagi S, Stasi C, Costa F, Mumolo MG, Ricchiuti A, Marchi S. Gastrointestinal manifestations in myotonic muscular dystrophy. World J Gastroenterol. 2006; 8;12(12):1821-8.

9. Reitsma JB, Rutjes AWS, Whiting P, VIssov VV, Leeflang MMG, Deeks JJ. Chapter 9: Assessing methodological quality. In: Deeks JJ, Bossuyt PM, Gatsonis C (editors), Cochrane Handbook for Systematic Reviews of Diagnostic Test Accuracy Version 1.0.0. The Cochrane Collaboration, 2009. http:/srdta.cochrane.org/.

10. Whiting P, Rutjes AWS, Reistma JB, Bossuyt P, Kleijnen J. The development of QUADAS: a tool for the quality assessment of studies of diagnostic accuracy included in systematic reviews. BMC Med Res Methodol. 2003;3;25.

11. Costantini M, Zaninotto G, Anselmino M, Marcon M, lurilli V, Boccu C, Feltrin G.P, Angelini C, Ancona E. Esophageal motor function in patients with myotonic dystrophy. Digest Dis Sci. 1996;41(10):2032-38.

12. Desuter G, Beguin C, Piessevaux H, Goncette L, Van Parijs V, Van Den Bergh P. Physiologic variables for videofluoromanometric assessment of dysphagia: An exploratory study. Acta Gastroenterol Belg. 2009; 72(3):312-20.

13. Eckardt VF, Nix W, Kraus W, Bohl J. Esophageal motor function in patients with muscular dystrophy. Gastroenterology. 1986;90(3):628-35.

14. Ertekin C, Yuceyar N, Aydogdu I, Karasoy H. Electrophysiological evaluation of oropharyngeal swallowing in myotonic dystrophy. J Neurol Neurosurg Psychiat. 2001;70(3):363-71.

15. Johnson ER, McKenzie SW. Pharyngeal abnormalities in myopathy: A videofluoroscopic study of swallow in polymyositis, limb-girdle muscular dystrophy and myotonic dystrophy. Phonoscope. 1999;2(4): 187-92.

16. Leonard RJ, Kendall KA, Johnson R, McKenzie S. Swallowing in myotonic muscular dystrophy: A videofluoroscopic study. Arch Phys Med Rehab. 2001;82(7):979-85.

17. Modolell I, Mearin F, Baudet JS, Gamez J, Cervera C, Malagelada JR Pharyngo-esophageal motility disturbances in patients with myotonic dystrophy. Scand J Gastroenterol. 1999;34(9):878-82.

18. Odman C, Kiliaridis S. Masticatory muscle activity in myotonic dystrophy patients. Journal of Oral Rehabilitation. 1996;23(1):5-10.

19. Ronnblom A, Forsberg H, Danielsson A. Gastrointestinal symptoms in myotonic dystrophy. Scand J Gastroenterol.1996;31(7):654-57.

20. Chiappetta ALML, Oda AL, Zanoteli E, Guilherme A, Oliveira, ASB. Oropharyngeal disphagia in the myotonic dystrophia: phonoaudiological evaluation and nasofibrolaryngoscopical analysis. Arq.Neuropsiquiatr. 2001;59(2-B):394-400.

21. Johnson ER, McKenzie SW. Kinematic pharyngeal transit times in myopathy: evaluation for dysphagia. Dysphagia. 1993;8(1):35-40.

22. Marcon M, Briani C, Ermani M, Menegazzo E, lurilli V, Feltrin GP, Novelli G, Gennarelli M, Angelini C. Positive correlation of CTG expansion and pharyngoesophageal alterations in myotonic dystrophy patients. Ital J Neurol Sci. 1998;19(2):75-80. 
23. Ramírez AF, Aguirre AU, Ancira FP, Mata MR. Esophageal motility abnormalities in myotonia dystrophica. Rev Invest Clin. 1981;33(1):25-8.

24. St. Guily JL, Perie S, Willig TN, Chaussade S, Eymard B, Angelard B. Swallowing disorders in muscular diseases: Functional assessment and indications of cricopharyngeal myotomy. Ear, Nose and Throat J. 1994;73(1):34-40.

25. Swick HM, Werlin SL, Dodds WJ, Hogan WJ. Esophageal motor function in patients with muscular dystrophy. Gastroenterology. 1986;90(3):628-35.

26. Willig TN, Paulus J, Guily JL.S, Beon C, Navarro J. Swallowing problems in neuromuscular disorders. Archives of Physical Medicine and Rehabilitation. 1994;75(11):1175-81. 



\title{
Chapter 3
}

Swallowing assessment in myotonic dystrophy type 1 using fiberoptic endoscopic evaluation of swallowing

(FEES)

\author{
Walmari Pilz \\ Laura W.J. Baijens \\ Valéria Lima Passos \\ Rob Verdonschot \\ Frederik Wesseling \\ Nel Roodenburg \\ Catharina G Faber \\ Bernd Kremer
}

Neuromuscul Disord. 2014 Dec;24(12):1054-62 


\section{Abstract}

This study describes the swallowing function of patients with myotonic dystrophy type 1 (DM1) and the effect of bolus consistency on swallowing in this group. The aim of the study is two-fold: (a) to identify which (and to what extent) swallowing variables change for DM1 patients relative to healthy control subjects and (b) to examine whether the degree of oropharyngeal dysphagia is associated with disease severity. Forty-five consecutive DM1 patients and ten healthy subjects underwent a swallowing assessment, at Maastricht University medical Center in the Netherlands. The assessment included a standardized fiberoptic endoscopic evaluation of swallowing (FEES) protocol using different bolus consistencies. Clinical severity of the disease was assessed using the Muscular Impairment Rating Scale (MIRS). Significant differences were found between patients and controls for all FEES variables. The magnitude of these differences depended on the bolus consistency. The odds of a more pathological swallowing outcome increased significantly with higher MIRS levels. In conclusion, swallowing function is found to be significantly altered in DM1 patients. The results emphasize the importance of conducting a detailed swallowing assessment in all patients, even those with mild muscle weakness. 


\section{Introduction}

Myotonic dystrophy type 1 (DM1) is the most common form of muscular dystrophy in adults. Its main clinical features are myotonia and weakness of facial, axial, and distal muscles. ${ }^{1}$ Besides its neuromuscular symptoms, DM1 has many other systemic manifestations, notably endocrine dysfunction and cardiac conduction defects. ${ }^{1}$ Though progressing slowly, the disease can be extremely disabling, especially in later stages as weakness becomes generalized and respiratory muscles are affected. ${ }^{2}$ The association with dysphagia leads to a high mortality rate in this population due to pneumonia and respiratory failure, whereby the median age at death is 56 years for patients with the adult type of DM1., Oropharyngeal dysphagia, although highly prevalent in DM1 patients, has received little attention in the literature. A safe and reliable method to evaluate it is fiberoptic endoscopic evaluation of swallowing (FEES). ${ }^{5,6}$ FEES permits anatomical assessment of the pharyngeal structures and provides a comprehensive evaluation of the pharyngeal stage of swallowing. Even though FEES has been recommended by many, few studies have described its application in DM1 patients. ${ }^{7,8}$ There is no curative treatment for DM1, but patients' quality of life can be improved by managing the symptoms. Complications such as aspiration may be prevented by swallowing treatment, which usually entails changes in food consistency to improve swallowing safety. ${ }^{9}$ However, reports on the impact of different consistencies in the swallowing performance of DM1 are scant. To help fill that gap, this study describes the swallowing function of DM1 patients and the effects of bolus consistency on swallowing safety. The aim of the study is twofold: (a) to identify which (and to what extent) swallowing variables change for DM1 patients relative to healthy control subjects and (b) to examine whether the degree of oropharyngeal dysphagia is associated with disease severity.

\section{Methods}

\section{Participants}

All DM1 patients were referred to the multidisciplinary outpatient clinic for dysphagia in the Maastricht University Medical Center (MUMC) by their neurologist as part of the regular health care program for these patients. Their diagnosis was based on genetic analysis. The neuromuscular involvement of the disease was scored by a neurologist with the muscular impairment rating scale (MIRS). ${ }^{10}$ The MIRS scale consists of 5 levels: (1) no muscular impairment; (2) minimal signs: myotonia, jaw and temporal wasting, facial weakness, neck flexor weakness, ptosis, nasal speech; (3) digital weakness; (4) mild to moderate proximal weakness; and (5) severe proximal weakness. The Body Mass Index (BMI) was calculated for all patients. The healthy subject group comprised staff members from the MUMC and volunteer students. They did not have any 
complaints of dysphagia, a neurological disease, craniofacial abnormalities or a gastrointestinal disease. The patient sample and control group were not age and gender matched. Individuals were excluded on the following grounds: if they had any other neurological disease (besides DM1); were over eighty years old (presbyphagia); were unable to perform a swallow (aphagia); suffered from severe mental depression, severe cognitive impairment, or head and neck cancer; had received speech therapy during the past six months; or had undergone surgery of the swallowing mechanism or the central nervous system. Informed consent was obtained from all patients. The study protocol was approved by the medical ethics committee of the MUMC.

\section{Swallowing assessment}

All measurements were performed by the same multidisciplinary team. The laryngologist and the speech and language pathologist were blinded to disease duration and data on the neurologic evaluation. Dietary intake was assessed using the Functional Oral Intake Scale (FOIS) (Table 3.1). ${ }^{11}$ All subjects underwent a standardized FEES examination. ${ }^{12}$ FEES allows the dysphagia clinician to directly visualize the pharyngeal and laryngeal structures and their function before and after swallowing. ${ }^{12}$ During FEES, the subjects were seated upright and had to swallow the bolus after it had been accurately delivered by syringe in the oral cavity. Three consistencies were administered: three trials of $10 \mathrm{cc}$ of thin liquid (water dyed with $5 \%$ methylene blue); three trials of $10 \mathrm{cc}$ of thick liquid (applesauce dyed with $5 \%$ methylene blue); and one solid (a bite-sized cracker). All participants swallowed the bolus consistencies in the same sequence (thin liquid, thick liquid, and bite-sized cracker). The tip of the flexible fiberoptic endoscope Pentax FNL-10RP3 (Pentax Canada Inc., Mississauga, Ontario, Canada) was positioned just above the epiglottis. Neither a nasal vasoconstrictor nor a topical anesthetic had been administered to the nasal mucosa. FEES images were obtained using an Alphatron Stroboview ACLS camera, Alphatron Lightsource, IVACX computerized video archiving system (Alphatron Medical Systems, Rotterdam, The Netherlands) and recorded on a DVD.

Table 3.1 Functional Oral Intake Scale (FOIS)

\begin{tabular}{cl}
\hline $\begin{array}{c}\text { Severity level of impaired dietary } \\
\text { intake }\end{array}$ & Definition \\
\hline 1 & Nothing by mouth \\
2 & Tube dependent with minimal attempts of food or liquid \\
3 & Tube dependent with consistent oral intake of food or liquid \\
4 & Total oral diet of a single consistency \\
5 & Total oral diet with multiple consistencies, requiring special \\
& preparation or compensations \\
6 & $\begin{array}{l}\text { Total oral diet with multiple consistencies without special } \\
\text { preparation, but with specific food limitation } \\
\text { Total oral diet with no restrictions }\end{array}$ \\
\hline
\end{tabular}




\section{FEES variables (outcome measures)}

Before the experiment was started, two raters received consensus training for the visuoperceptual measurement of ordinal-scale variables (Table 3.2). They practiced the exact interpretation per level of each of the scales. A written manual with well-defined descriptions of the scales' levels was available during the training session and rating process. Visuoperceptual ordinal variables were scored for each FEES swallow at varying speed (slow motion, normal, up to frame-by-frame speed) as often as necessary, using the software program Windows Movie Maker version 5.1 (Microsoft Corporation, Redmond, WA, USA). The raters were blinded to the patients' medical history, the participants' group (patients vs. controls), and to each other's scores (independent rating). The swallow studies were scored in randomized order. Furthermore, the raters were advised to limit the duration of the measurement sessions (max. two hours). To obtain intrarater reliability, each rater repeated the measurement of 57 swallows within a period of two weeks.

Table 3.2 Description of the visuoperceptual FEES variables

\begin{tabular}{|c|c|c|}
\hline Name & Definition & Rating scale \\
\hline Piecemeal deglutition & Sequential swallowing of the same bolus & Five-point scale (0-4) \\
\hline Delayed initiation pharyngeal reflex & Delayed onset pharyngeal triggering & Three-point scale (0-2) \\
\hline Postswallow vallecular pooling & $\begin{array}{l}\text { Bolus retention in the vallecula after } \\
\text { swallowing }\end{array}$ & Three-point scale (0-2) \\
\hline Postswallow pyriform pooling & $\begin{array}{l}\text { Bolus retention in the pyriform sinuses } \\
\text { after swallowing }\end{array}$ & Three-point scale (0-2) \\
\hline $\begin{array}{l}\text { Laryngeal penetration and/or } \\
\text { tracheal aspiration }\end{array}$ & $\begin{array}{l}\text { Material in the laryngeal vestibule above } \\
\text { or on the level of the vocal folds and/or } \\
\text { material below the vocal folds }\end{array}$ & Three-point scale (0-2) \\
\hline
\end{tabular}

\section{Statistical analysis}

Reliability analysis was performed using a weighted kappa index of agreement (intrarater and interrater) for all visuoperceptual ordinal FEES variables. The multilevel structure of the data, with measurements nested within subjects (i.e., repeated swallows), induced correlation among the observations. To warrant the assumption of independence, kappas were computed separately per swallow. Unadjusted group comparisons were made by means of non-parametric tests (Kruskal-Wallis) for age and of Fisher's exact tests for sex. Consistent with the two-pronged objective of the study, multivariable analyses of the FEES outcome were conducted twice: first, for all data, to compare the patients and healthy controls; and second, for patient data only, to examine specific changes in swallowing as a function of disease severity. For both runs, the selection of the statistical model was determined by the nature of the FEES measurement scales (categorical vs. continuous). Multinomial logistic regression models with random intercept were selected for the trichotomous categorical variables (delayed initiation of the pharyngeal reflex, postswallow vallecular pooling, postswallow pyriform sinus pooling, laryngeal penetration, and tracheal aspiration). For 
the continuous FEES variable (piecemeal deglutition), multilevel analysis was conducted with the linear random intercept regression model. The objective of the multinomial logistic regression was to model the odds for classification of subjects in the pathological modalities of each trichotomous outcome variable, whereas the objective of the linear approach was to evaluate the average changes of piecemeal deglutition. Either way, changes in FEES outcome variables were modeled as a function of group (patients vs. controls), bolus consistency, and, when applicable, the number of swallow trials, with concurrent correction for age, rater, and sex. These variables comprised the fixed effect part of the model. Subjects comprised the random effect, which was introduced to capture the multilevel structure of the data. For the second part of the analysis (patient data only), an extra variable, MIRS, was introduced in order to test whether the swallowing function is influenced by the level of muscle impairment while adjusted for disease duration. Interaction terms, which were deemed clinically meaningful, were added to the model: e.g., interaction between group (patients vs. controls) and bolus consistency and between MIRS and bolus consistency (patient data). When failing to reach statistical significance, the interaction terms were dropped; if significant, stratified analyses followed. All models were fitted with PROC GLIMMIX in SAS, version 9.2 (SAS Institute Inc., Cary, NC, U.S.A.).

\section{Results}

\section{Reliability results}

Intra- and interrater reliability were sufficient for all FEES variables (weighted kappa $>0.61)$.

\section{Characteristics of the participants}

Forty-five consecutive DM1 patients and 10 healthy control subjects were included in the study. Their characteristics are presented in Table 3.3. The main dysphagia complains (if present) reported by the patients during the interview were: increased duration of mealtime, coughing while drinking, choking on foods etc. Given the sparse cell counts of MIRS 2 and 3 (none in MIRS 1), these categories were collapsed prior to drawing statistical inferences.

Table 3.3 Characteristics of the participants

\begin{tabular}{|c|c|c|c|c|c|}
\hline & MD Group $(n=45)$ & & Healthy Control Group ( $n=$ & & $P$ value $^{\text {a }}$ \\
\hline \multirow[t]{2}{*}{ Male / Female } & $28 / 17$ & & $3 / 7$ & & .148 \\
\hline & Median (25th, 75th perc) & Range & Median (25th, 75th perc) & Range & $P$ value \\
\hline Age & $46(38,55)$ & $21-69$ & $29(16,50)$ & $19-60$ & 0.047 \\
\hline BMI & $25(23,30)$ & $15-45$ & NA & NA & NA \\
\hline Disease duration & $14(4,23)$ & $1-41$ & NA & NA & NA \\
\hline MIRS & $5(4,5)$ & $2-5$ & NA & NA & NA \\
\hline
\end{tabular}

${ }^{a}$ Significance level of $P<0.05 ; N A=$ not applicable 


\section{Comparison of patients and healthy control subjects}

Bolus consistency significantly affected swallowing among the patients but not among the healthy control subjects. This difference was confirmed by the multivariable analyses, in which interaction terms between group and bolus consistency were found to be statistically significant. Group comparisons were therefore conducted for each bolus consistency separately. Once adjusted for sex, age, and rater, statistically significant group differences (patients vs. controls) were observed for all visuoperceptual FEES variables using thin and thick liquid consistencies ( $P$ values $<0.001$ ) showing an impaired swallowing function in the patients. The difference between the groups for the variable 'piecemeal deglutition' was also significant at the bolus consistency of a bite-sized cracker. Despite significant group differences in the distribution of all FEES parameters, the odds ratios (ORs) of patients with respect to healthy control subjects are omitted, as most of the ORs were too large or infinite due to the presence of 'empty classes'. This may be explained as follows. Because none of the healthy control subjects had been assigned to the most severe level of swallowing impairment in their FEES outcome, the estimate of the odds for observing these severe scores (in healthy controls) was too small or zero. As a result, the ORs for patients with respect to healthy control subjects were large or infinite and the corresponding confidence intervals (Cls) were inflated. ${ }^{13}$ This problem was observed for almost all trichotomous FEES variables in the patients vs. controls comparison; the exceptions were the variables 'penetration' and 'piecemeal deglutition'. For penetration, the ORs (patients vs. controls) were computed with healthy control subjects taken as a reference. The ORs and 95\% Cls were 40.74 [3.42, 197.07] and 86.00 [12.25, 604.40] for thin and thick liquids respectively. Thus, despite the occurrence of penetration among healthy control subjects, this phenomenon was still significantly more frequent in DM1 patients. For piecemeal deglutition, patients had significantly higher mean values than healthy control subjects. When comparing the two groups (patients vs. controls), a means difference and Cls were found for each consistency as follows: 0.56 [0.17, 0.95] for thin liquid; 1.27 [0.90, 1.64] for thick liquid; and 1.63 [0.46, 5.87] for a solid (a bitesized cracker). These results show that the groups' average differences (patients vs. controls) in piecemeal deglutition also depended on the bolus consistencies, as the difference between the means for each of the groups is bigger for a bite-sized cracker than for thin liquids.

\section{Patient analyses}

\section{Effects of bolus consistency on the swallowing function of DM1 patients}

\section{Descriptive statistics}

The frequency distributions show that swallowing impairment levels varied not only among the FEES variables but also within each variable as a function of the bolus 
consistency (Figure 3.1 and 3.2). For instance, severe delayed initiation of the pharyngeal reflex and aspiration occurred more frequently in the swallows of thin liquid; in contrast, for postswallow vallecular pooling, postswallow pyriform sinus pooling, and piecemeal deglutition, patients were more often classified as severely impaired when swallowing thick liquids. Aspiration of thin liquid was observed in 17 of the 45 patients. Eleven of these 17 did not aspirate during the intake of thick liquid. Aspiration of thick liquid exclusively was observed in two of the 45 patients.
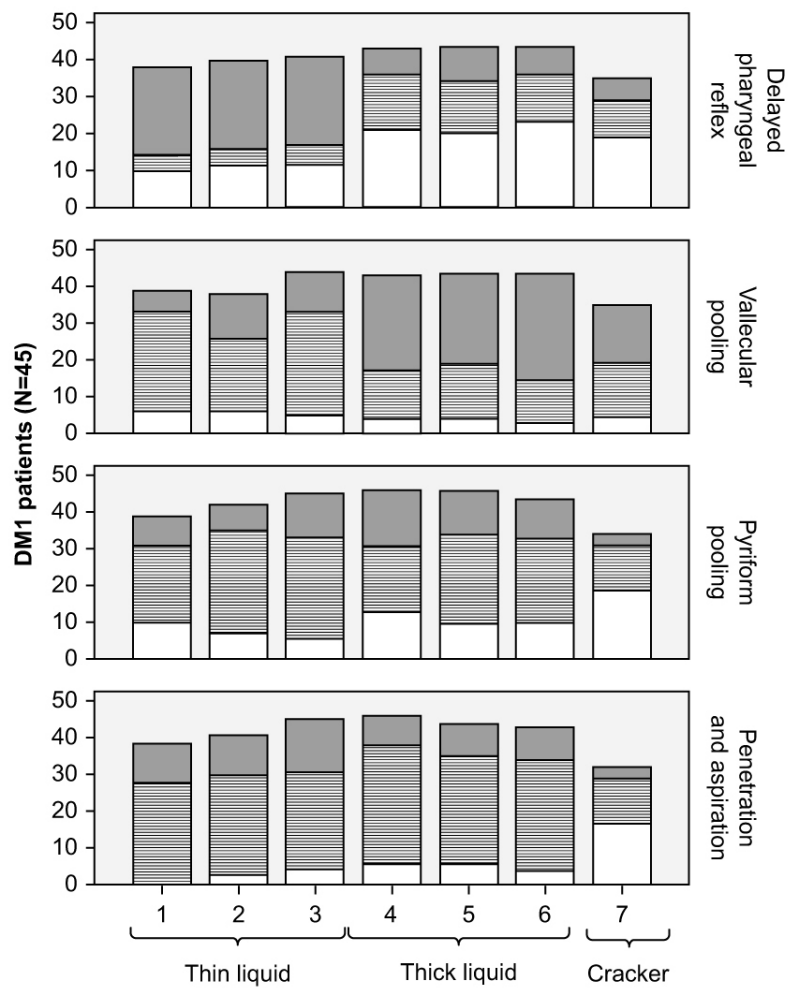

$\square$ Severe impairment 目 Moderate impairment $\square$ No impairment

Figure 3.1 Frequency distributions per swallow trial. The $y$-axis represents the frequency of occurrence (in absolute numbers) of patients in the ordinal-scale levels (0-2) of each of the FEES variables. The $x$-axis represents the number of swallow trials for each consistency. Note: For the variable 'penetration and aspiration', penetration was represented as moderate impairment and aspiration as severe impairment. 

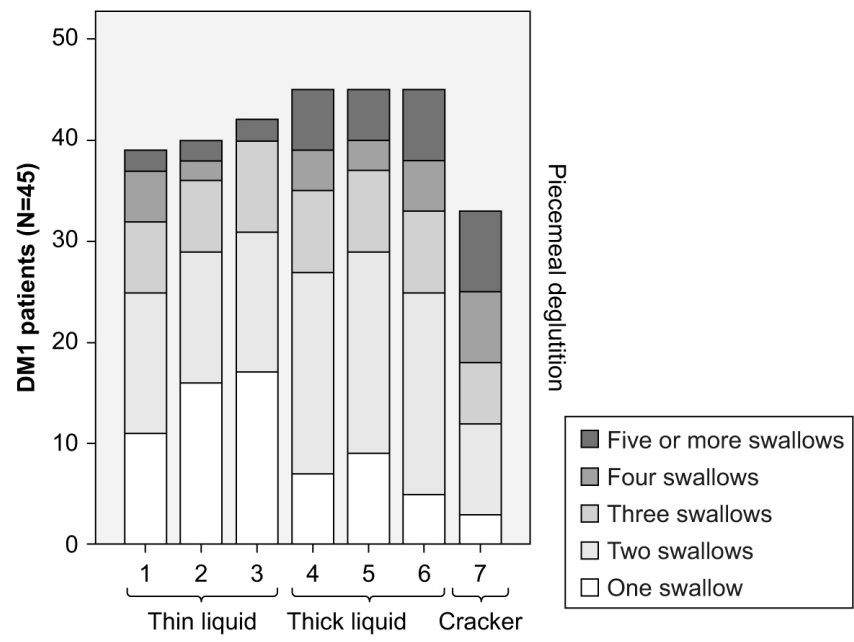

Figure 3.2 Frequency distributions of piecemeal deglutition per swallow trial. The $y$-axis represents the frequency of occurrence (in absolute numbers) of patients in the ordinal-scale levels (0-4) of the FEES variable 'piecemeal deglutition'. The $x$-axis represents the number of swallow trials for each consistency

Multivariable analysis

Figure 3.3 shows how the likelihood for a patient to be classified in the two levels of swallowing impairment (mild and severe impairment) relative to normal swallowing had changed as a function of the bolus consistency. All ORs were adjusted for the covariates (sex, age, rater). The comparison of bolus consistencies for the variable 'piecemeal deglutition' is presented in Figure 3.4. Interactions between disease duration and MIRS as well as between bolus consistency and MIRS were nonsignificant. However, for the FEES variable 'delayed initiation of the pharyngeal reflex', an interaction was found: a bolus consistency effect was significant for patients classified in MIRS level 5. For all FEES outcome variables, the likelihood to observe mild or severe swallowing impairment was significantly influenced by bolus consistency. Patients were more likely to present with mild or severe impairment when swallowing thin liquid than thick liquid $(O R s>1)$. This finding was consistent throughout many bolus consistency comparisons, with the exception of the variables 'postswallow vallecular pooling', 'piecemeal deglutition', and 'delayed initiation of pharyngeal reflex' (for MIRS 5 and mild swallowing impairment only); for these three variables, the patients showed more severe impairment when swallowing thick liquid than thin. Patients performed better (i.e., had less impaired FEES outcomes) with the solid bolus consistency than with the other consistencies, except for the variable 'piecemeal deglutition' (Figure 3.4). 


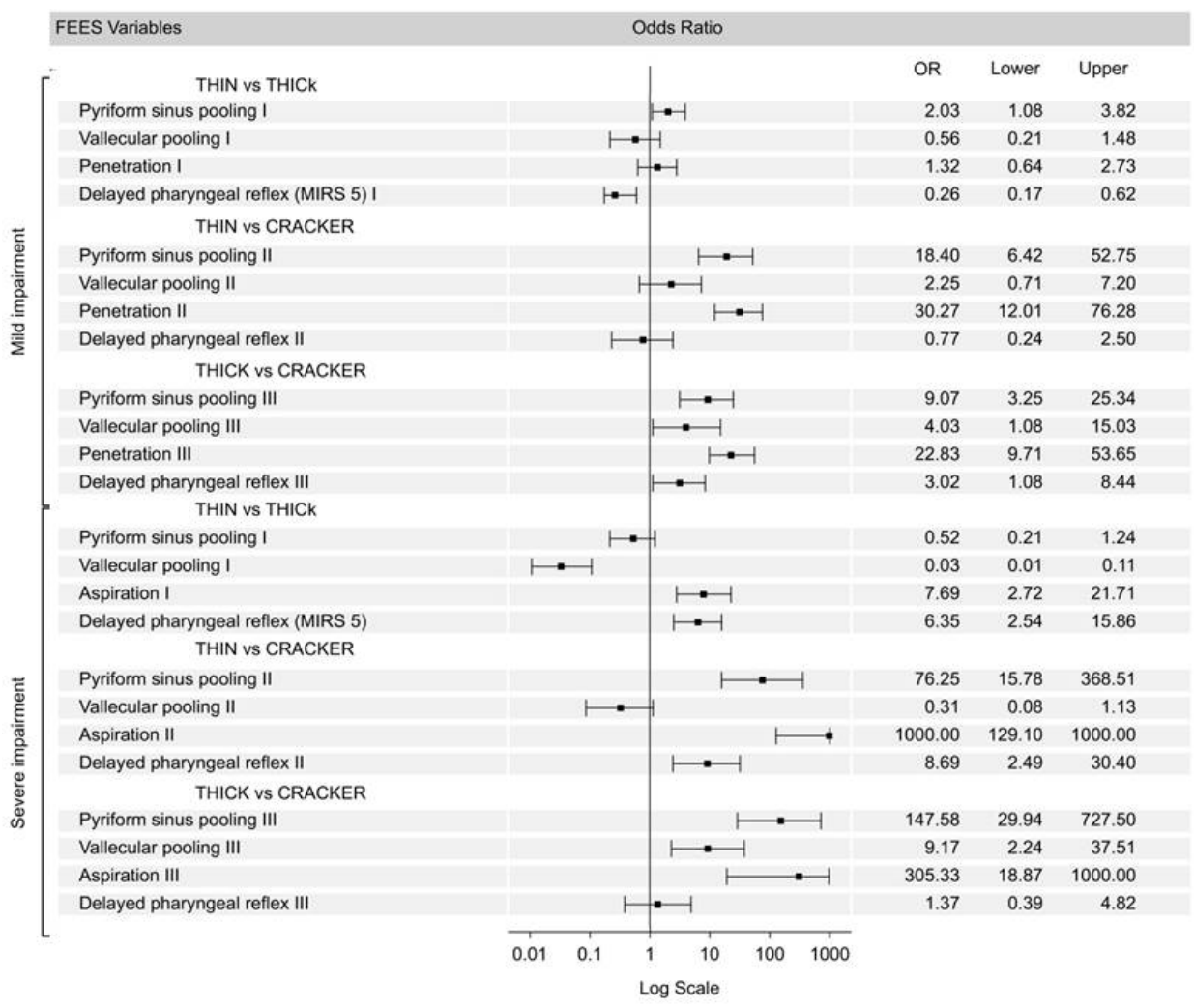

Figure 3.3 Effects of bolus consistency on trichotomous categorical FEES variables (patient data only). Two categories are shown: mildly impaired and severely impaired FEES variables. In both categories, ORs are supplied for the following comparisons of consistency: thin vs. thick liquid; thin liquid vs. solid (bite-sized cracker); and thick liquid vs. solid. To facilitate the interpretation of the forest plot, one comparison is selected for more detailed description. In the category 'severely impaired FEES variables', the point estimate OR for postswallow pyriform sinus pooling II comparing thin liquid to bite-sized cracker is 76.25 . This indicates that the odds of being allocated to the most severe impairment level of the scale, relative to normal swallowing, are increased almost 76-fold for swallows with thin liquid with respect to those swallows with a bite-sized cracker. This increase is statistically significant, as the $95 \% \mathrm{Cl}$ does not include the ' 1 '

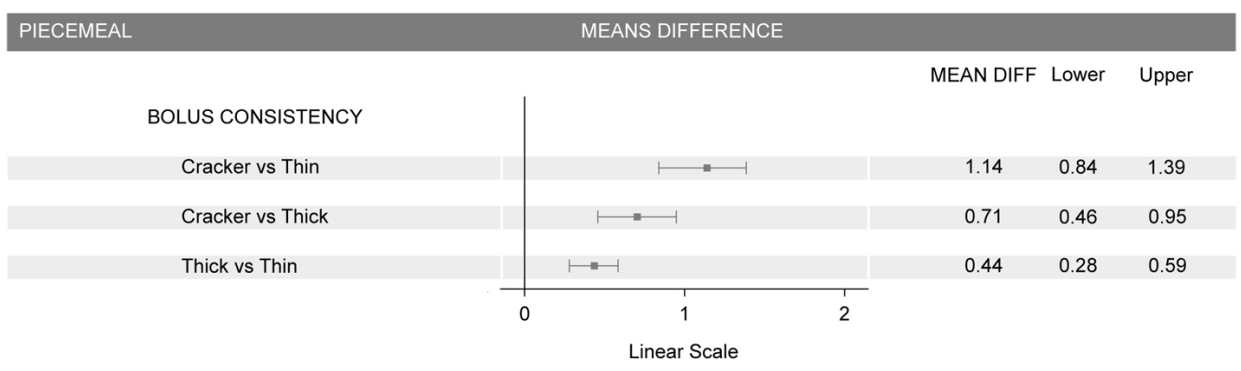

Figure 3.4 Effects of bolus consistency on the FEES variable 'piecemeal deglutition' (latently continuous variable). The $95 \%$ confidence interval of the means difference covering the vertical line ' 0 ' indicates a lack of statistical significance 


\section{FEES variables and MIRS score}

Figure 3.5 displays how the likelihood for a patient to be classified as having a mild swallowing impairment, relative to normal swallowing, changed as a function of disease severity (for all consistencies together). When comparing patients with MIRS 5 vs. MIRS 2 and 3 or with MIRS 5 vs. MIRS 4, significant differences were found for two FEES variables, 'postswallow vallecular pooling' and 'delayed initiation of the pharyngeal reflex', though found in the latter only for swallows of thick liquid consistency. These variables showed significantly more severe impairment in MIRS 5 (Figure 3.5). However, no significant differences in the swallowing performance between patients with MIRS 2 and 3 and patients with MIRS 4 could be detected. Furthermore, when comparing patients in the early stages of the disease (MIRS 2 and 3) with healthy control subjects, significant group differences were observed for all FEES variables. The ORs of the FEES variables representing severe swallowing impairment vs. no swallowing impairment were omitted; they could not be estimated due to the presence of empty classes, as reported previously.

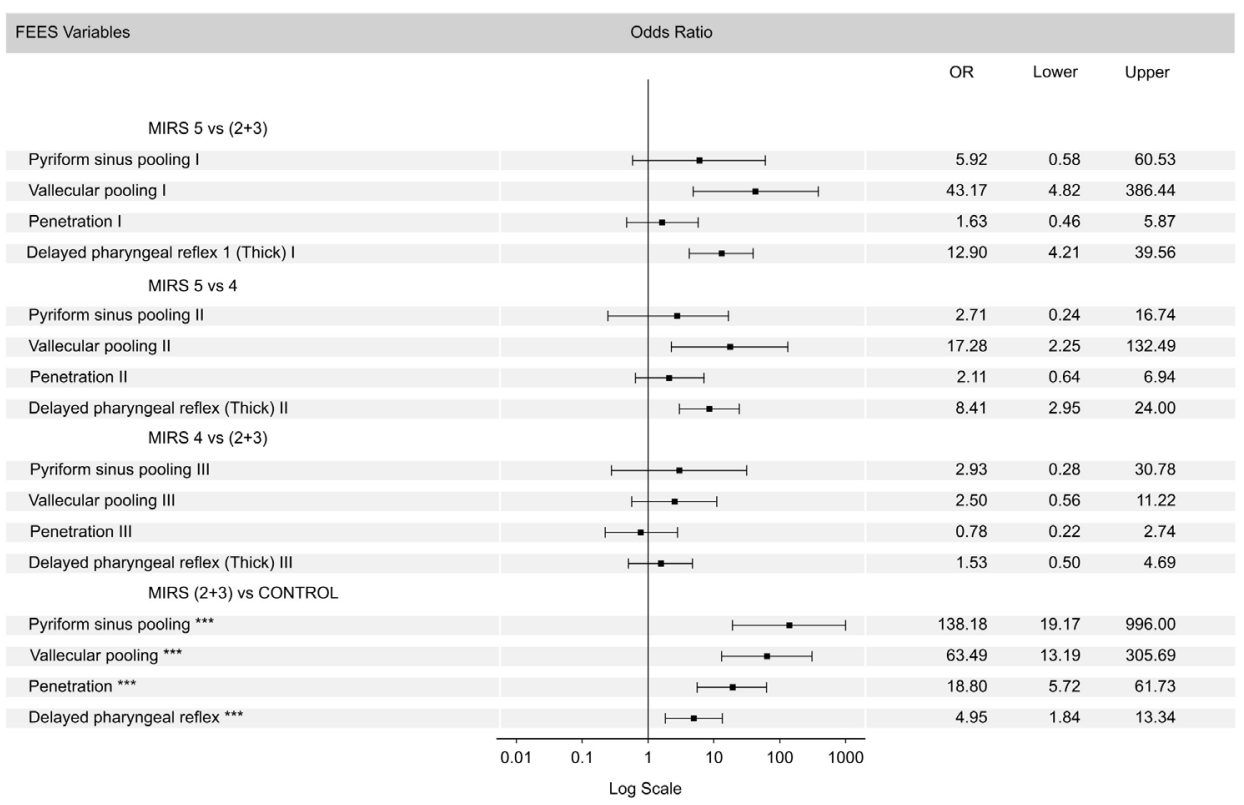

Figure 3.5 FEES variables and MIRS score. The ORs of the trichotomous categorical FEES variables represent mild swallowing impairment vs. no swallowing impairment as a function of the compared MIRS levels (for all consistencies together). Note: Estimated ORs are adjusted for age, sex, disease duration, rater and bolus consistency 


\section{Discussion}

This study shows that dysphagia is common in DM1 patients. Its presence is probably underestimated in this population as patients do not usually report swallowing complaints unless specifically questioned. ${ }^{14-16}$ This underreporting is well represented by the FOIS values. Although swallowing function was clearly impaired in most of the patients, none of them scored FOIS values of 4 (total oral diet of a single consistency) or less. Apparently, the adaptation of food consistency to facilitate swallowing is not common practice. Furthermore, patients seem to modulate swallowing function, as the disease progresses slowly. Adaptive changes in swallowing function, varying cognitive function among patients, non-complaining nature of patients, and their lack of initiative may influence the perception of swallowing difficulties. ${ }^{17}$ That may explain the discrepancy between the presence of dysphagia and the way swallowing impairment is either handled or ignored by the patients.

Although malnutrition and low body weight are frequent findings in patients presenting with oropharyngeal dysphagia, only one DM1 patient in this study had a BMI $<18$. The high number of obese DM1 patients ( $N=9$ (20\%); BMI>30) has been described previously. $^{18}$ This finding might reflect the metabolic syndrome associated with DM1 disease. The weight gain in these patients might also be attributed to progressive muscle loss with fat infiltration, reduced mobility, and a sedentary lifestyle. ${ }^{18}$

On all FEES outcome variables, the patients were scored as mildly to severely impaired. Postswallow pharyngeal (vallecular and pyriform sinus) pooling and piecemeal deglutition seem to be important determinants of oropharyngeal dysphagia in DM1. These findings are substantiated by previous investigations. Studies using manometry to evaluate swallowing in DM1 report a decreased pharyngeal contraction amplitude that may cause pharyngeal pooling. ${ }^{16,19}$ Postswallow pharyngeal pooling and piecemeal deglutition appear to be closely linked with each other during swallowing in DM1. It is hypothesized that piecemeal deglutition can be generated in response to pharyngeal pooling, supporting the findings of a study with neurogenic dysphagic patients. ${ }^{20}$ Nonetheless, piecemeal deglutition can also be the result of an ineffective oral transit due to weaknesses of the facial, oral, and masticatory muscles in DM1. ${ }^{21}$

As expected, aspiration occurred more often with thin liquid than with other consistencies. Swallowing of thin liquids requires higher speed and more precise coordination of swallowing than needed for other consistencies. ${ }^{22}$ Moreover, in DM1 patients, the oral motor movements are usually slow and uncoordinated, causing a delayed motor response following the onset of swallowing. ${ }^{23}$ As a result, the risk of aspiration of the bolus, and consequently the development of pneumonia, increases. Pneumonia and respiratory problems are known to be the most frequent causes of death in DM1, ranging from $31 \%$ to $43 \%$. $^{3,4}$

The current study shows that bolus consistency affected FEES outcomes in DM1 patients in different ways. For most FEES outcome variables, patients exhibited a higher level of impairment for swallows with thin liquids than with thick liquids. However, for 
the solid consistency (bite-sized cracker), the scores on most FEES variables were better than for other bolus consistencies. It was already reported that DM1 patients need more time and more chewing cycles to prepare a solid bolus for swallowing. ${ }^{24}$ Chewing longer may give them more time to prepare the pharyngeal structures to receive the bolus, and this could explain why patients performed better when swallowing a bitesized cracker. Compared to thick liquid, the solid consistency led to less postswallow vallecular pooling; still, sixteen of the 45 patients did present with severe postswallow vallecular pooling when given a bite-sized cracker. This indicates that swallowing a bitesized cracker can be a challenge to some DM1 patients.

Furthermore, the study results highlight the need to assess the effects of bolus consistency on the swallowing function of DM1 patients, as consistency may influence both diagnostic outcomes and treatment planning. While bolus modification has often been used in daily clinical practice to promote swallowing safety in dysphagic DM1 patients, few scientific investigations have examined the effects. ${ }^{7}$ As most of the patients in the current study aspirated thin liquid only, procedures to thicken liquids as a therapeutic strategy can be considered but should be carefully applied.

According to the current findings, dysphagia is already present in the early stages (MIRS levels 2 and 3 ) of the muscular disease. However, swallowing dysfunction and muscular impairment do not develop synchronously. After an initial decrease, the swallowing function seems to stabilize and only deteriorates in the final stage of the disease. Nevertheless, not all FEES variables were affected in the same way; delayed initiation of the pharyngeal reflex (thick liquids), aspiration, and postswallow vallecular pooling were significantly associated with progression of the disease.

\section{Methodological limitations}

To avoid methodological shortcomings, several precautions were taken. For example, all measurements were performed in the same hospital by the same multidisciplinary team in order to standardize the data collection. The median MIRS score of 5 for the participants may have led to results with less external validity. Still, the study population was a reflection of the theoretical population of dysphagic DM1 patients who are referred by the neurologist or speech and language pathologist for swallowing evaluation. Due to the relatively small sample size, data were sparsely distributed, resulting in ORs that were either unreliable or impossible to estimate. Although a dichotomization of the FEES variables (impaired vs. not impaired) might have prevented wide Cls, it would have caused a loss of information that was deemed clinically meaningful.

In conclusion, FEES is well tolerated by the DM1 patients. The FEES outcomes reported here show that swallowing function is significantly altered in DM1 patients and that the magnitude of the impairment depends on, among other factors, the bolus consistency. The results emphasize the importance of a detailed swallowing assessment such as FEES in all patients, even in those with mild muscle weakness. An early detection of 
oropharyngeal dysphagia may prevent commonly associated health complications such as aspiration pneumonia and may help to improve quality of life in these patients. 


\section{References}

1. Harper PS. Myotonic dystrophy. London: Saunders, 2001.

2. Hermans MC, Faber CG, De Baets MH, de Die-Smulders CE, Merkies IS. Rasch-built myotonic dystrophy type 1 activity and participation scale (DM1-Activ). Neuromuscul Disord. 2010;20(5):310-8.

3. Mathieu J, Allard P, Potvin L, Prévost C, Bégin P. A 10-year study of mortality in a cohort of patients with myotonic dystrophy. Neurology. 1999;52(8):1658-62.

4. de Die-Smulders CE, Höweler CJ, Thijs C, et al. Age and causes of death in adult-onset myotonic dystrophy. Brain.1998; 121(8):1557-63.

5. Langmore SE, Schatz K, Olsen N. Fiberoptic endoscopic examination of swallowing safety: a new procedure. Dysphagia. 1988;2(4):216-9.

6. Leder SB, Murray JT. Fiberoptic endoscopic evaluation of swallowing. Phys Med RehabilClin N Am. 2008; 19(4):787-801

7. Chiappetta ALML, Oda AL, Zanoteli E, Guilherme A, Oliveira, ASB. Oropharyngeal dysphagia in the myotonic dystrophia: phonoaudiological evaluation and nasofibrolaryngoscopical analysis (in Portuguese). Arq Neuropsiquiatr. 2001;59(2-B):394-400.

8. St. Guily JL, Perie S, Willig TN, Chaussade S, Eymard B, Angelard B. Swallowing disorders in muscular diseases: Functional assessment and indications of cricopharyngeal myotomy. Ear, Nose and Throat J. 1994; 73(1):34-40.

9. Hill M, Hughes T, Milford C. Treatment for swallowing difficulties (dysphagia) in chronic muscle disease. Cochrane Database Syst Rev. 2004;(2):CD004303.

10. Mathieu J, Boivin H, Meunier D, Gaudreault M, Bégin P. Assessment of a disease-specific muscular impairment rating scale in myotonic dystrophy. Neurology. 2001;56(3):336-40.

11. Crary MA, Carnaby Mann GD, Groher ME. Initial psychometric assessment of a functional oral intake scale for dysphagia in stroke patients. Arch Phys Med Rehabil. 2005;86(8):1516-20.

12. Langmore SE, Aviv JE. Endoscopic procedures to evaluate oropharyngeal swallowing. In: Langmore SE, ed. Endoscopic Evaluation and Treatment of Swallowing Disorders. New York, NY, USA: Thieme, 2001; 73-100.

13. Irala J, Navajas RFC; Navajas ASC, Castillo AS. Abnormally wide confidence intervals in logistic regression: interpretation of statistical program results. Pan Am J Public Health 1997;2(4):268-71.

14. Leonard RJ, Kendall KA, Johnson R, McKenzie S. Swallowing in myotonic muscular dystrophy: A videofluoroscopic study. Arch Phys Med Rehabil. 2001;82(7):979-85.

15. Eckardt VF, Nix W, Kraus W, Bohl J. Esophageal motor function in patients with muscular dystrophy. Gastroenterology. 1986;90(3):628-35.

16. Modolell I, Mearin F, Baudet JS, Gamez J, Cervera C, Malagelada JR Pharyngo-esophageal motility disturbances in patients with myotonic dystrophy. Scand J Gastroenterol. 1999;34(9):878-82.

17. Meola G, Sansone V. Cerebral involvement in myotonic dystrophies. Muscle Nerve. 2007;36(3):294-306.

18. Pruna L, Chatelin J, Pascal-Vigneron V, Kaminsky P. Regional body composition and functional impairment in patients with myotonic dystrophy. Muscle Nerve. 2011;44(4):503-8.

19. Costantini M, Zaninotto G, Anselmino M, et al. Esophageal motor function in patients with myotonic dystrophy. Digest Dis Sci. 1996;41(10):2032-8.

20. Molfenter SM, Steele CM. The relationship between residue and aspiration on the subsequent swallow: an application of the normalized residue ratio scale. Dysphagia. 2013;28(4):494-500.

21. Ertekin C, Yuceyar N, Aydogdu I, Karasoy H. Electrophysiological evaluation of oropharyngeal swallowing in myotonic dystrophy. J Neurol Neurosurg Psychiat. 2001;70(3):363-71.

22. Kendall KA, Leonard RJ, McKenzie SW. Accommodation to changes in bolus viscosity in normal deglutition: a videofluoroscopic study. Ann Otol Rhinol Laryngol. 2001;110(11):1059-65.

23. Ercolin B, Sassi FC, Mangilli LD, Mendonça LI, Limongi SC, de Andrade CR. Oral motor movements and swallowing in patients with myotonic dystrophy type 1. Dysphagia. 2013;28(3):446-54.

24. Odman C, Kiliaridis S. Masticatory muscle activity in myotonic dystrophy patients. J Oral Rehabil. 1996; 23(1):5-10. 



\section{Chapter 4}

\section{Swallow-related quality of life AND oropharyngeal dysphagia in myotonic dystrophy}

Walmari Pilz

Valéria Lima Passos

Rob J. Verdonschot

Jolanda Meijers

Nel Roodenburg

Catharin G. Faber

Bernd Kremer

Laura W. Baijens

Submitted, under revision 


\section{Abstract}

This study describes swallow-related quality of life (SWAL-QOL) in patients with myotonic dystrophy type 1 (DM1) and investigates its association with swallowing function and disease severity. A SWAL-QOL questionnaire was completed by 75 DM1 patients and 25 healthy control subjects. The severity of the disease was evaluated using the muscular impairment rating scale (MIRS). Twenty-eight DM1 patients underwent a videofluoroscopic swallowing examination (VFS). Spearman's correlation coefficient was used to measure the direction and strength of associations. The SWAL-QOL median scores were significantly lower for the DM1 group than for the healthy control group. The correlation between disease severity and SWAL-QOL was significant in four domains. Postswallow vallecular pooling and piecemeal deglutition were the most impaired VFS outcome variables. Our results suggest that a multidimensional swallowing assessment is recommended for DM1 patients as SWAL-QOL and VFS measure different aspects of the swallowing function, thus providing complementary information. 


\section{Introduction}

Myotonic dystrophy type 1 (DM1) is the most common form of muscular dystrophy in adults and is dominantly inherited. The clinical manifestations, which are highly variable, include myotonia, muscular dystrophy, cataracts, and involvement of other organs such as the heart, endocrine system, and brain. In addition, cognitive dysfunction, reduced initiative, inactivity, and apathy are consistent with the disease, and these characteristics have healthcare implications for DM1 patients. ${ }^{1}$ Fatigue and reduced mobility have been identified as the symptoms most disturbing to their daily life. $^{2}$ Moreover, since the weakness present in many parts of the body also affects oropharyngeal muscles, swallowing impairment is prevalent in $\mathrm{DM} 1$. $^{3,4}$

DM1 patients report lower QoL than healthy subjects and these scores were associated to fatigue, daytime sleepiness, and muscular impairment. ${ }^{5,6}$ Although these prior studies have evaluated the influence of its physical and psychological manifestations on health-related quality of life (QoL), little is known about the impact of swallowing impairment on health-related QoL in DM1 patients. Besides fulfilling a basic need, eating has significant psychological and social functions. An evaluation of swallowing should therefore cover not only its physiological aspects but should also take patients' perception into account. To that end, data would have to be gathered on the impact of swallowing impairment on health-related QoL. A better understanding of how DM1 patients perceive and cope with oropharyngeal dysphagia could improve the guidelines for clinical interventions and rehabilitation programs. From that perspective, the aim of this study was twofold: (a) to describe swallow-related QoL in dysphagic patients with DM1, and (b) to investigate the relationship between swallow-related QoL with swallowing function on the one hand and disease severity on the other hand.

\section{Methods}

\section{Participants}

Genetically confirmed DM1 patients were prospectively included in this study and were consecutively recruited from the multidisciplinary outpatient clinic for dysphagia in the Maastricht University Medical Center (MUMC). Their diagnosis was based on genetic analysis. All recruited patients were referred for swallowing examination on the grounds of signs or symptoms of deglutition disorders, i.e., cough while eating, sensation of food getting stuck in the throat, long time to finish a meal, etc. Individuals were excluded if they had any other neurological disease (besides DM1) or head and neck cancer; had received speech therapy in the past (to exclude benefit of treatment and attention) had cognitive impairment (MMSE (mini mental state examination) <23); or had undergone surgery of critical structures involved in swallowing (tongue, larynx, etc.) or the central nervous system. Patients over eighty years old were excluded due to 
the possibility of presenting dysphagia as a result of aging (presbyphagia); As it is not known precisely when presbyphagia begins, the age of eighty years was arbitrarily chosen as a cutoff point. Healthy participants without swallowing complaints were recruited from the local community to serve as a control group. Informed consent was obtained from all participants. The study protocol was approved by the medical ethics committee of the MUMC.

\section{Measures}

\section{Disease severity}

The neuromuscular involvement of the disease was scored by a neurologist using the muscular impairment rating scale (MIRS). ${ }^{7}$ This scale expresses a clinical assessment of the progression of muscular impairment (distal to proximal) in DM1. It classifies the degree of impairment in an ordinal scale ranging from one (no muscular impairment) to five (severe proximal weakness).

\section{Swallow-related QoL}

The SWAL-QOL survey was designed to evaluate the impact of swallowing problems on the health-related QoL of patients with oropharyngeal dysphagia. ${ }^{8}$ The questionnaire was translated into the Dutch language and then validated. ${ }^{9}$ The SWAL-QOL was designed to assess eight domains of swallow-related QoL (general burden, food selection, eating duration, eating desire, fear of eating, communication, social functioning, and mental health), two concepts of generic QoL (fatigue and sleep), and a dysphagia clinical symptom scale (symptom score). The score of each domain is calculated based on two or more questions. The score per domain ranges from 0 (extremely impaired) to 100 (no impairment).

\section{Swallowing function}

Swallowing function was evaluated by performing a videofluoroscopic swallowing study (VFS). During the VFS, the patients were offered one trial of thin liquid (low-density barium - 40\% w/v(weight/volume) and one trial of thick liquid (50 cc applesauce +150 gr barium powder) followed by one bite-sized cracker coated with barium paste. All measurements followed the same protocol. ${ }^{10}$ Each participant swallowed the bolus consistencies upon command and in the same sequence (thin liquid, thick liquid, and bite-sized cracker). The VFS was performed in a lateral position. The images were obtained with a Philips Diagnost 97 system (Philips Medical Systems, Eindhoven, The Netherlands) and recorded on DVD at 30 frames per second. All swallows were analyzed by two experienced raters trained in the VFS scoring system. They scored four ordinal variables (piecemeal deglutition, postswallow vallecular pooling, postswallow pyriform pooling, and laryngeal penetration and aspiration). ${ }^{11}$ The raters were blinded to each other's results (independent rating) and to the patients' medical history. A 
written manual with well-defined descriptions of the scales' levels was available during the rating process. The swallows were scored in randomized order at varying speed (slow motion, normal, up to frame-by-frame speed) using the software program Windows Movie Maker version 5.1 (Microsoft Corporation, Redmond, WA, USA). The raters were advised to limit the duration of the measurement sessions (max. two hours) to avoid fatigue. To obtain intrarater agreement, each rater repeated the measurement of 39 swallows within a period of two weeks. The scores of the rater with the highest indices of intrarater agreement were used for subsequent statistical analysis.

\section{Statistical analysis}

Raters' agreement was analyzed with a weighted Kappa (for ordinal variables). The internal consistency of the SWAL-QOL was analyzed using Cronbach's alpha. The participants' characteristics are presented as absolute numbers/percentages for categorical variables and median and 25th; 75th percentiles for continuous variables, due to data skewness. Spearman's correlation was used to measure the direction and strength of correlations. The two groups (DM1 patients and healthy control subjects) were compared with the Mann-Whitney $U$ test. The internal consistency of the SWALQOL was analyzed using Cronbach's alpha. Significance level was $5 \%$. Because of the large number of null-hypothesis tests conducted, especially the many pair-wise correlations, p-values were adjusted for multiple-testing with False Discovery Rate (FDR) correction.

\section{Results}

\section{Characteristics of the participants}

SWAL-QOL was completed by 25 healthy control subjects and 75 DM1 patients. The mean age in the control group was 45.6 years, ranging from 22 to 71 , and $52 \%$ were men. In the patient group, the mean age was 43.43, ranging from 21 to 73 , and 55\% were men. Most DM1 patients presented mild to moderate proximal muscular weakness (MIRS 4, N=22) or severe proximal muscular weakness (MIRS 5, N=23). Eight patients presented minimal signs of muscular impairment (MIRS 2).

All healthy control subjects and over $68 \%$ of the patients completed the questionnaire by themselves. The other $32 \%$ of the patients received help, mainly with reading the questions and/or writing the answers. Most of the patients reported having a normal diet without texture modification. 


\section{SWAL-QOL}

The scores of all SWAL-QOL domains were significantly lower for the DM1 group than for the healthy control group (Table 4.1). In the healthy control group most of the SWAL-QOL domains showed a skewed distribution with an accentuated ceiling effect. No floor effect was observed. In the patients group, three SWAL-QOL domains presented a higher percentage of ceiling effect: 'social functioning' (56\%), 'eating desire' (48\%), and 'fear of eating' (48\%). The dysphagia symptoms most frequently reported in the study were: 'coughing', 'having to clear the throat', and 'food sticking in the throat'. No correlations were found between age, gender, and each of the eleven SWAL-QOL domains. Patients reporting decreased general health had significant lower scores in all SWAL-QOL domains. The correlations ranged from rho $=0.253$ ('eating desire') to rho $=0.480$ ('dysphagia symptom').

Table 4.1 Differences on SWAL-QOL domains between DM1 patients and healthy subjects

\begin{tabular}{lcccc}
\hline SWAL-QOL domains & $\begin{array}{c}\text { DM1 patients } \\
\mathrm{N}=75\end{array}$ & $\begin{array}{c}\text { Healthy control } \\
\text { subjects } \\
\mathrm{N}=25\end{array}$ & $\mathrm{p}$-value $^{\mathrm{b}}$ & ${\text { Cronbach's } \alpha^{\mathrm{c}}}$ \\
\hline Burden & $88(63 ; 100)^{1}$ & $100(100 ; 100)^{\mathrm{a}}$ & 0.001 & 0.90 \\
Food selection & $75(50 ; 100)$ & $100(100 ; 100)$ & 0.001 & 0.83 \\
Eating duration & $50(25 ; 88)$ & $100(94 ; 100)$ & 0.001 & 0.85 \\
Eating desire & $92(58 ; 100)$ & $100(100 ; 100)$ & 0.047 & 0.87 \\
Fear of eating & $94(75 ; 100)$ & $100(100 ; 100)$ & 0.001 & 0.84 \\
Sleep & $75(50 ; 100)$ & $88(75 ; 100)$ & 0.217 & 0.84 \\
Fatigue & $42(25 ; 75)$ & $92(83 ; 100)$ & 0.001 & 0.88 \\
Communication & $75(63 ; 88)$ & $100(100 ; 100)$ & 0.001 & 0.83 \\
Mental health & $95(75 ; 100)$ & $100(100 ; 100)$ & 0.001 & 0.88 \\
Social functioning & $100(75 ; 100)$ & $100(100 ; 100)$ & 0.017 & 0.94 \\
Symptom score & $66(54 ; 86)$ & $100(95.5 ; 100)$ & 0.001 & 0.94 \\
\hline
\end{tabular}

a Values are median and $25^{\text {th }} ; 75^{\text {th }}$ percentiles; ${ }^{\text {b }}$ Mann-Whitney $U$ test ; Significance level: $p$-value $<0.05$;

${ }^{\mathrm{c}}$ Cronbach's $\alpha>0.7$

\section{VFS measurements in the DM1 group}

The VFS outcome measurements of the first subset of 28 DM1 patients were analyzed. Twenty of these patients were male and the mean age was 46.5 years. MIRS ranged from 1 to 5 , median 5 (25th; 75th percentiles: 3.25 ; 5). Intra- and interrater agreement indices were substantial to almost perfect for all measured VFS variables. Analysis of the visuoperceptual variables revealed that swallowing function was impaired in the majority of the patients. The variable showing the highest level of impairment was postswallow vallecular pooling (Figure 4.1). The percentages of patients with a score of two or more on the variable piecemeal deglutition were $46.1 \%$ (thin liquid), $48.1 \%$ (thick liquid), and $66.6 \%$ (cracker). One patient aspirated thin liquid and two patients aspirated both thin and thick liquid. 


\section{Relationship between disease severity, SWAL-QOL, and VFS measurements in the DM1 group}

The scores for the majority of the SWAL-QOL domains were lower in patients with proximal muscular weakness (MIRS 4 and 5). However, when comparing the score of each SWAL-QOL domain between the muscular impairment severity levels (MIRS 1 to $5)$, the differences did not reach statistical significance. Spearman's correlation coefficient between severity of muscular impairment (MIRS) and SWAL-QOL showed a weak correlation in four domains: 'general burden' (rho=-0. 241), 'eating duration' (rho=-0.287), 'fear of eating' (rho=-0.275), and 'symptom score' (rho=-0.322). Two VFS outcome measurements correlated with MIRS: piecemeal deglutition of bite-sized cracker (rho=0.466) and postswallow pyriform sinus pooling of thick liquid (rho=0.376). Although the these correlations were significant in the preliminary statistical analyses, none of them reached the level of significance after FDR correction for multiple-testing.

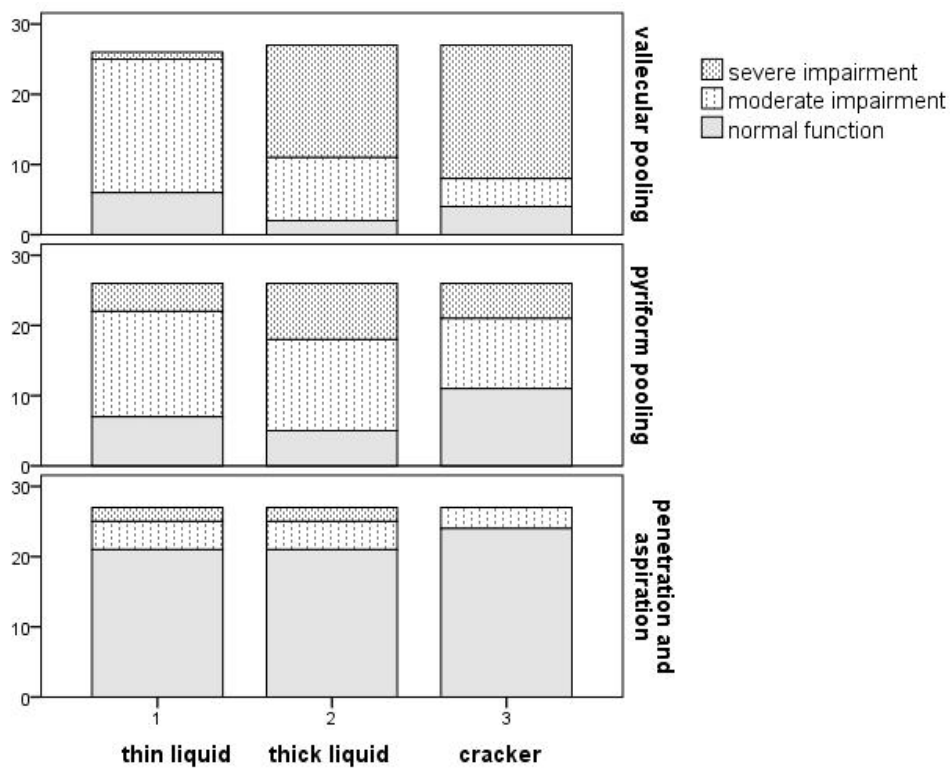

Figure 4.1 Frequency distribution of DM1 patients $(\mathrm{N}=28)$ per consistency on three VFS outcome variables. The $y$-axis represents the frequency of occurrence in absolute numbers of patients. The $x$-axis indicates the consistency of each swallow.

Note: For the variables 'vallecular and pyriform sinus pooling', trace of pooling up to filling of less than $50 \%$ of the vallecular space/pyriform sinus is represented as moderate impairment. Filling of more than $50 \%$ of the vallecular space/pyriform sinus is represented as severe impairment. For the variable 'penetration and aspiration', penetration is represented as moderate impairment and aspiration as severe impairment 


\section{Relationship between VFS measurements and SWAL-QOL in the DM1 group}

The SWAL-QOL domains 'general burden', 'eating duration', 'eating desire', and 'fear of eating', showed moderate correlation with VFS outcome measurements, though inconclusive after FDR correction (Table 4.2).

Table 4.2 Correlation between SWAL-QOL domains and VFS outcomes. The level of significance ( $p$ ) before and $\operatorname{after}\left({ }^{*}\right)$ the correction for multiple tests is presented

\begin{tabular}{|c|c|c|c|c|c|}
\hline \multicolumn{2}{|l|}{ VFS } & \multicolumn{4}{|c|}{ SWAL-QOL } \\
\hline & & 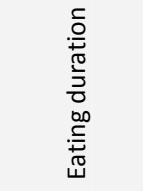 & 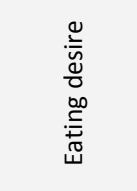 & 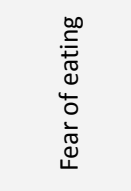 & 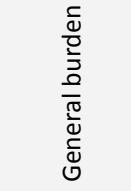 \\
\hline 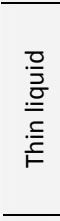 & $\begin{array}{l}\text { Postswallow vallecular pooling } \\
\text { Postswallow pyriform sinus pooling }\end{array}$ & $\begin{array}{c}-0.438 \\
p=0.012 \\
p=0.193^{*}\end{array}$ & $\begin{array}{c}0.484 \\
p=0.025 \\
p=0.123^{*}\end{array}$ & & \\
\hline 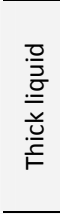 & $\begin{array}{l}\text { Postswallow vallecular pooling } \\
\text { Postswallow pyriform sinus pooling }\end{array}$ & & $\begin{array}{c}-0.453 \\
p=0.020 \\
p=0.228^{*}\end{array}$ & $\begin{array}{c}-0.410 \\
p=0.034 \\
p=0.180^{*}\end{array}$ & \\
\hline 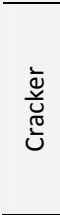 & $\begin{array}{l}\text { Postswallow pyriform sinus pooling } \\
\text { Piecemeal deglutition }\end{array}$ & $\begin{array}{c}-0.478 \\
p=0.012 \\
p=0.123^{*}\end{array}$ & & & $\begin{array}{c}0.532 \\
p=0.005 \\
p=0.064^{*}\end{array}$ \\
\hline
\end{tabular}

\section{Discussion}

This study describes swallowing-related QoL in DM1 patients and evaluates the correlation between patient-reported impact of dysphagia on QoL, instrumental assessment of the swallowing function, and disease severity. The differences in the SWAL-QOL scores between the healthy control group and DM1 patients were significant and are in concordance with previous studies reporting lower SWAL-QOL scores in dysphagic subjects. ${ }^{8,12}$ Conversely, correlations between VFS outcome measurements, on the one hand, and SWAL-QOL domains and disease severity, on the other, were inconclusive after multiple testing correction. 
In the DM1 group, the SWAL-QOL domain with the lowest scores (representing more impairment) was 'fatigue', followed by 'eating duration', and 'symptom score'. The high frequency of fatigue found in this study is not surprising and is consistent with results from other studies. ${ }^{2,13,14}$ In the domain 'eating duration', lower scores representing a longer amount of time to eat a meal were frequently found. A longer duration may be linked to weakness of the oral and pharyngeal muscles in DM1 patients [154]. Moreover, weak oropharyngeal muscles can impair the propulsion of the bolus from the mouth to the esophagus. An ineffective bolus propulsion usually generates repeated swallows in a physiological attempt to clear the residue from the oral cavity and pharynx. ${ }^{16}$ As a consequence, a frequent occurrence of repeated swallows or 'piecemeal deglutition' may extend the duration of the meal.

Disease severity has been considered one of the main factors of health-related QoL in patients with neuromuscular disease. The impact of the progression of the disease on various aspects of health-related QoL, other than swallowing, has already been reported. ${ }^{5,6,17}$ Our results showed a weak relationship between the severity of muscular impairment and SWAL-QOL domains as well as the severity of swallowing impairment.. The hypothesis that the severity of the muscular impairment would have an impact on the SWAL-QOL domains should be further investigated in a longitudinal study.

This study analyzed a correlation between SWAL-QOL domains and VFS outcomes measurements to ascertain whether patients' perception of the impact of dysphagia on QoL directly reflects the severity of the swallowing impairment. The moderate correlation between these two assessment approaches, lacking statistical significance after adjustment for multiple testing, suggests that patients with an impaired swallowing function not always report a decrease in their swallow-related QoL. Besides instrumental swallowing assessment using VFS the intrinsic characteristics of a tool that evaluated the impact of swallowing impairment on QoL and the variety of aspects involved in the eating process should also be considered. SWAL-QOL scores are based on patients' personal perspective on the impact of dysphagia and are not a direct reflection of their physiological swallowing ability. Social, behavioral, and psychological aspects are entangled when feeding is self-assessed, and patients have different levels of perception and tolerance when facing a physical limitation. Especially in a disease with a slow progression such as DM1, patients adapt their actions and behavior, so their attitude can influence the scores on SWAL-QOL. In that light, our study confirms in the DM1 population the findings from a previous study that investigated a broad variety of dysphagic patients: SWAL-QoL and VFS measure different aspects or dimensions of the swallowing function, thus providing complementary information. ${ }^{18}$ Moreover, it reinforces the importance of a multidimensional swallowing assessment, including an instrumental examination, such as fiberoptic endoscopic evaluation of swallowing (FEES) and VFS, in all DM1 patient with dysphagia.

SWAL-QOL provides valuable information on how these patients perceive and cope with their swallowing disability. This information is essential for rehabilitation programs, as it can guide the clinicians' approach when proposing changes in food 
consistencies or an exercise program. Knowing which QoL aspects are impacted by dysphagia allow clinicians to look beyond the swallowing function, focusing on interventions that would have an effect on QoL. For instance, as fatigue and duration of the meals were SWAL-QOL domains affected by dysphagia in DM1 patients, the rehabilitation plan should include strategies to shorten the time patients spend eating a meal. Strategies such as, bolus modification, changes in food portion sizes, frequency of meals, etc. The success of a rehabilitation program is dependent on patients' commitment, and changes in eating behavior are only possible if patients recognize the severity of their disease and the limitations imposed by their swallowing problem.

\section{Methodological limitations}

The present study has some methodological limitations that should be taken into consideration. Though the DM1 patients scored significantly lower than the control group and all patients reported one or more dysphagia symptoms, most SWAL-QOL domains were not scored as severely impaired. The homogeneity of the SWAL-QOL scores, especially those with ceiling effects, is bound to have attenuated statistical correlations due to the restriction of range effect.

\section{Conclusion}

SWAL-QOL provides information on how DM1 patients perceive their swallowing impairment and on the impact of dysphagia on their swallow-related QoL. There was no correlation between the severity of muscular impairment on one hand and VFS outcome and SWAL-QOL scores on the other. Interestingly, the weak to moderate, but eventually inconclusive correlations between VFS and SWAL-QOL outcomes suggests that these two tools measure different aspects of dysphagia in DM1 patients. If that is indeed the case, SWAL-QOL should not be taken as an indicator of the severity of dysphagia for these patients. Instead, a multidimensional swallowing assessment protocol is recommended for DM1 patients. 


\section{References}

1. Meola G, Sansone V. Cerebral involvement in myotonic dystrophies. Muscle Nerve. 2007;36(3):294-306.

2. Heatwole C, Bode R, Johnson N, Quinn C, Martens W, McDermott MP, et al. Patient-reported impact of symptoms in myotonic dystrophy type 1 (PRISM-1). Neurology. 2012;79(4):348-57.

3. Costantini M, Zaninotto G, Anselmino M, et al. Esophageal motor function in patients with myotonic dystrophy. Digest Dis Sci. 1996;41(10):2032-8.

4. Pilz W, Baijens LW, Passos VL, et al. Swallowing assessment in myotonic dystrophy type 1 using fiberoptic endoscopic evaluation of swallowing (FEES). Neuromuscul Disord. 2014;24(12):1054-62.

5. Peric S, Stojanovic VR, Basta I, et al. Influence of multisystemic affection on health-related quality of life in patients with myotonic dystrophy type 1. Clin Neurol Neurosurg. 2013;115(3):270-5.

6. Laberge L, Mathieu J, Auclair J, Gagnon É, Noreau L, Gagnon C. Clinical, psychosocial, and central correlates of quality of life in myotonic dystrophy type 1 patients. Eur Neurol. 2013;70(5-6):308-15.

7. Mathieu J, Boivin H, Meunier D, Gaudreault M, Bégin P. Assessment of a disease-specific muscular impairment rating scale in myotonic dystrophy. Neurology. 2001;56(3):336-40.

8. McHorney CA, Robbins J, Lomax K, et al. The SWAL-QOL and SWAL-CARE outcomes tool for oropharyngeal dysphagia in adults: III. Documentation of reliability and validity. Dysphagia. 2002;17(2):97-114.

9. Bogaardt HCA, Speyer R, Baijens LWJ, FokkensWJ. Cross-cultural Adaptation and Validation of the Dutch Version of SWAL-QOL. Dysphagia. 2009;24:66-70.

10. Baijens LW, Speyer R, Passos VL, Pilz W, Roodenburg N, Clavé P. The effect of surface electrical stimulation on swallowing in dysphagic Parkinson patients. Dysphagia. 2012;27(4):528-37.

11. Baijens LW, Speyer R, Passos VL, Pilz W, van der Kruis J, Haarmans S, Desjardins-Rombouts C. Surface electrical stimulation in dysphagic Parkinson patients: a randomized clinical trial. Laryngoscope. 2013; 123(11):E38-44

12. Vanderwegen J, Van Nuffelen G, De Bodt M. The validation and psychometric properties of the Dutch version of the Swallowing Quality-of-Life Questionnaire (DSWAL-QOL). Dysphagia. 2013;28(1):11-23.

13. Rakocevic-Stojanovic V, Peric S, Madzarevic R, et al. Significant impact of behavioral and cognitive impairment on quality of life in patients with myotonic dystrophy type 1. Clin Neurol Neurosurg. 2014; 126:76-81

14. Hermans MC, Faber CG, De Baets MH, de Die-Smulders CE, Merkies IS. Rasch-built myotonic dystrophy type 1 activity and participation scale (DM1-Activ). Neuromuscul Disord. 2010;20(5):310-8.

15. Umemoto G, Nakamura H, Oya Y, Kikuta T. Masticatory dysfunction in patients with myotonic dystrophy (type 1): a 5-year follow-up. Spec Care Dentist. 2009;29(5):210-4.

16. Ertekin C, Aydoğdu I, Yüceyar N. Piecemeal deglutition and dysphagia limit in normal subjects and in patients with swallowing disorders. J Neurol Neurosurg Psychiatry. 1996;61(5):491-6.

17. Graham CD, Rose MR, Grunfeld EA, et al: A systematic review of quality of life in adults with muscle disease. J Neurol 2011;258: 1581-92.

18. McHorney CA, Martin Harris B, Robbins J, Rosenbek J.Clinical validity of the SWAI-QOL and SWAL-CARE outcome tools with respect to bolus flow measures. Dysphagia 2006;21(3):141-8. 



\section{Chapter 5}

\section{Observers' agreement on measurements in fiberoptic endoscopic evaluation of swallowing}

Walmari Pilz

Sophie Vanbelle

Bernd Kremer

Michel R. van Hooren

Tine van Becelaere

Nel Roodenburg

Laura W.J. Baijens

Dysphagia. 2016 Apr;31(2):180-7 


\section{Abstract}

This study analyzed the effect that dysphagia etiology, different observers, and bolus consistency might have on the level of agreement for measurements in FEES images reached by independent versus consensus panel rating. Sixty patients were included and divided into two groups according to dysphagia etiology: neurological or head and neck oncological. All patients underwent standardized FEES examination using thin and thick liquid consistencies. Two observers scored the same exams, first independently and then in a consensus panel. Four ordinal FEES variables were analyzed. Statistical analysis was performed using a linear weighted kappa coefficient and Bayesian multilevel model. Intra- and interobserver agreement on FEES measurements ranged from 0.76 to 0.93 and from 0.61 to 0.88 , respectively. Dysphagia etiology did not influence observers' agreement level. However, bolus consistency resulted in decreased interobserver agreement for all measured FEES variables during thin liquid swallows. When rating on the consensus panel, the observers deviated considerably from the scores they had previously given on the independent rating task. Observer agreement on measurements in FEES exams was influenced by bolus consistency, not by dysphagia etiology. Therefore, observer agreement on FEES measurements should be analyzed by taking bolus consistency into account, as it might affect the interpretation of the outcome. Identifying factors that might influence agreement levels could lead to better understanding of the rating process and assist in developing a more precise measurement scale that would ensure higher levels of observer agreement for measurements in FEES exams. 


\section{Introduction}

Fiberoptic endoscopic evaluation of swallowing (FEES) has been widely used to evaluate oropharyngeal dysphagia since it was first described in $1988 .{ }^{1}$ Besides being safe and easy to use, FEES permits the anatomical assessment of the pharyngeal and laryngeal structures; it also constitutes a comprehensive evaluation of the pharyngeal stage of swallowing. ${ }^{2}$ For these reasons, both diagnosis and treatment planning of deglutition disorders often take FEES outcome measurements into account. While the popularity of FEES as an assessment tool is increasing, research on standardization and validation of measurement criteria in these exams lags behind. Crucially, interpretation of swallowing images is based on visual judgment and is thus subjective. It might be influenced by factors such as experience of the observer(s), bolus consistency, and dysphagia severity. ${ }^{3-5}$ Moreover, the literature on swallowing evaluation rarely describes the protocols or the variables analyzed in sufficient detail. ${ }^{6}$ A few studies have addressed observer agreement on some well-known visuoperceptual ordinal variables, such as the Penetration Aspiration Scale (PAS) and the pharyngeal residue scale. Nonetheless, the variability in the scoring of FEES exams remains underexplored. ${ }^{7-10}$ Given its role in clinical decision making, an accurate and reliable measurement technique is necessary.

In this paper, rather than simply report estimate agreement indexes, a statistical multilevel approach method was used to analyse the data. This method quantifies the impact of predictors, e.g., consistency, dysphagia etiology, etc., on observers' agreement and permits the identification of aspects influencing negatively the level of agreement. $^{11}$ By identifying factors that can influence observers' agreement on measured FEES variables, researchers can better understand the rating process and thereby help develop a procedure to increase observer agreement levels. In that light, the aim of this study is to compare (1) observers' agreement on FEES measurements in patients with dysphagia of neurological versus head and neck oncological origin, and (2) observers' behavior in independent versus consensus panel rating.

\section{Methods}

\section{Subject selection}

Thirty consecutive patients with dysphagia of neurological origin and thirty consecutive patients with dysphagia of head and neck oncological origin were included. All patients underwent FEES examination, from 2010 to 2012, in the Maastricht University Medical Center (MUMC). Oropharyngeal dysphagia was identified by the multidisciplinary team based on clinical assessment and FEES examination. Patients were excluded if they presented severe dyskinesia of the head and neck, suffered from severe mental 
depression, had cognitive impairment (Mini Mental State Examination score <23), or had concurrent head and neck cancer and a neurological disease.

\section{Swallowing assessment}

All measurements were performed in the same hospital by the same multidisciplinary team. All subjects underwent the same FEES protocol. ${ }^{12}$ During the exam, two consistencies were administered: three $10 \mathrm{cc}$ trials of thin liquid (water dyed with $5 \%$ methylene blue); and three $10 \mathrm{cc}$ trials of thick liquid (applesauce dyed with 5\% methylene blue). All participants were offered the bolus consistencies in the same sequence (thin liquid followed by thick). The tip of the flexible fiberoptic endoscope Pentax FNL-10RP3 (Pentax Canada Inc., Mississauga, Ontario, Canada) was positioned just above the epiglottis. Neither a nasal vasoconstrictor nor a topical anesthetic was administered to the nasal mucosa. Images were obtained using an Alphatron Stroboview ACLS camera, Alphatron Lightsource, and IVACX computerized video archiving system (Alphatron Medical Systems, Rotterdam, the Netherlands) and recorded on a DVD at 30 frames per second.

\section{Swallowing measurements}

Two students in their last year of medical school without experience in swallowing evaluation were selected as observers. Prior to data collection, they completed an intensive training program on the rating scales of four visuoperceptual ordinal variables (Table 5.1). The observers were jointly trained in the interpretation of the scales by an expert. A written manual with well-defined descriptions of the levels was available during the training program and the subsequent rating process and could be consulted anytime. The duration of the training program was pre-determined and consisted of 10 training sessions, of approximately $1 \mathrm{~h}$ each. The training sessions were interspersed with practice periods when the observers had to do test runs separately. Each practice period consisted of $2 \mathrm{~h}$, in average. The results were discussed in the next training session. All FEES exams selected were scored separately by an expert. During the training session, the exams were jointly analyzed and discussed between the observers and the expert. Moreover, observers' scores of the training session and the practice session were compared to expert scores to assess medical student's accuracy of FEES interpretation. The training was predominantly targeted to generate sufficient intraand interobserver agreement levels. After ten training sessions the statistical analyses of the practice trials showed sufficient interobserver agreement (weighted kappa $\geq 0.6$ ), so the observers were confident about starting to rate the FEES exams for the present study. All four visuoperceptual ordinal variables were scored for each deglutition. The entire recording of each swallowing act was analyzed at varying speed (slow motion, normal, and frame-by-frame) as often as necessary, using the software program Windows Movie Maker version 5.1 (Microsoft Corporation, Redmond, WA, USA). During training, an equal amount of FEES images was taken from each etiological group 
for analysis. The observers were blinded to the patients' medical history and the origin of their oropharyngeal dysphagia. The swallows were scored in random order. Furthermore, observers were advised to limit the duration of the measurement sessions to $2 \mathrm{~h}$ to avoid fatigue, which could introduce bias. The process was divided into two separate tasks: independent rating and consensus panel rating. When rating independently, the observers were blinded to each other's scores; on the consensus panel, the two observers analyzed the swallowing videos together and the scores were determined by consensus agreement. To reach intraobserver agreement, each observer performed repeated measurements independently within a period of two weeks. The consensus panel task was also repeated to obtain test re-test agreement. The number of swallows was balanced regarding bolus consistency (thin and thick liquid) and patient group (neurological and oncological origin) for all tasks.

Table 5.1 Description of the ordinal rating scales of the four visuoperceptual FEES variables

\begin{tabular}{|c|c|c|}
\hline FEES variable & Definition & Rating scale \\
\hline Piecemeal deglutition & Sequential swallowing on the same bolus & $\begin{array}{l}0=\text { one swallow } \\
1=\text { two swallows } \\
2=\text { three swallows } \\
3=\text { four swallows } \\
4=\text { five or more swallows }\end{array}$ \\
\hline $\begin{array}{l}\text { Postswallow vallecular } \\
\text { pooling }\end{array}$ & $\begin{array}{l}\text { Bolus retention in the valleculae after } \\
\text { swallowing }\end{array}$ & $\begin{aligned} 0= & \text { no pooling } \\
1= & \text { filling of less than } 50 \% \text { of the } \\
& \text { valleculae } \\
2= & \text { filling of more than } 50 \% \text { of the } \\
& \text { valleculae }\end{aligned}$ \\
\hline $\begin{array}{l}\text { Postswallow pyriform } \\
\text { sinus pooling }\end{array}$ & $\begin{array}{l}\text { Bolus retention in the pyriform sinuses } \\
\text { after swallowing }\end{array}$ & $\begin{aligned} 0= & \text { no pooling } \\
1= & \text { trace to moderate pooling } \\
2= & \text { severe pooling up to complete } \\
& \text { filling of the sinus. }\end{aligned}$ \\
\hline $\begin{array}{l}\text { Laryngeal penetration } \\
\text { /tracheal aspiration }\end{array}$ & $\begin{array}{l}\text { Bolus in the laryngeal vestibule above or } \\
\text { on the level of the vocal folds (laryngeal } \\
\text { penetration) or bolus passes below the } \\
\text { vocal folds (tracheal aspiration) }\end{array}$ & $\begin{array}{l}0=\text { no laryngeal penetration } \\
1=\text { laryngeal penetration } \\
2=\text { tracheal aspiration }\end{array}$ \\
\hline
\end{tabular}

\section{Statistical analysis}

Results were expressed as mean and standard error (SE) for quantitative variables, while frequencies and proportions (\%) were used for ordinal variables. The intra- and interobserver agreement was quantified using the linear weighted kappa coefficient. The weighted kappa values were interpreted as poor (0), slight $(0.00-0.20)$, fair (0.21$0.40)$, moderate (0.41-0.60), substantial (0.61-0.80), and almost perfect agreement $(0.81-1) .^{13}$ The standard error of weighted kappa coefficients was adjusted for the repeated measurements taken from the patients. ${ }^{14}$ The effect of predictors (dysphagia etiology, different observers, and bolus consistency) on the intra- and interobserver agreement levels and the probability of changing the FEES scores of the independent rating task during the consensus panel was analyzed using a multilevel approach. ${ }^{11}$ 
Random effects relative to the patients were introduced in the models to capture the multiple measurements for each patient (six swallows). The variance of these random effects is denoted by $\sigma^{2}$. Large values indicate heterogeneous agreement levels among patients while small values indicate homogeneous agreement levels. The intercept is used to give the average agreement levels for a median patient in all the reference categories (i.e., observer 2, thick liquid, neurological patient). A Bayesian approach was used to estimate the parameters in the model. In Bayesian estimation, the prior knowledge about parameters is combined with the observed data to yield a posterior distribution. Vague priors, which express that we do not have prior information on the parameters, were used. The posterior summary measures were obtained using the Markov-Chain Monte Carlo (MCMC) sampling approach. A predictor is said to be significant if the $95 \%$ equal-tailed posterior credibility interval relative to the predictor does not contain the value 0 . Data analysis was conducted using $R$ (version 3.0.2 for Windows) and WinBUGS statistical packages.

\section{Results}

\section{Characteristics of the subjects}

Sixty mentally competent dysphagic patients were included. Thirty had a diagnosis of neurological origin: myotonic dystrophy (14), stroke (4), Parkinson disease (3), amyotrophic lateral sclerosis (2), inclusion body myositis (2), myasthenia gravis (1), Duchenne muscular dystrophy (1), cerebellar syndrome (1), multiple sclerosis (1), and extra-pyramidal syndrome (1). The other thirty had a diagnosis of head and neck oncological origin: laryngeal carcinoma (10), oropharyngeal carcinoma (9), oral cavity carcinoma (5), nasopharyngeal carcinoma (3), hypopharyngeal carcinoma (2), and parotid gland carcinoma (1). All oncological patients completed treatment at least three months prior to the FEES examination and none of the patients was in a palliative state of care. The mean age in the neurological group was 57 (SE: 3.21); in the oncological group it was 65 (SE: 2.04). The level of swallowing impairment represented by FEES scores was similar for both groups (Table 5.2). The exception was the variable laryngeal penetration/tracheal aspiration, for which the oncological group presented significantly higher scores, indicating more severe impairment.

\section{Number of swallows analyzed}

In total 360 swallows were recorded (six swallows per patient). Two observers scored all 360 independently within a period of 3 months. From these, 120 swallows were randomly selected and scored by both observers also in a consensus panel setting within a period of 3 weeks. To investigate intraobserver agreement, the two observers independently repeated the measurement of 80 randomized swallows within a period 
of 2 weeks. For the test re-test agreement of the consensus panel the observers repeated the measurement of 40 randomized swallows within a period of 1 week.

Table 5.2 Frequency distribution of patients per category of the different FEES variables, given as absolute numbers $N$ and percentages (\%) according to the etiological group

\begin{tabular}{lccc}
\hline & & \multicolumn{2}{c}{ Etiology } \\
\hline FEES variables & Rating scale & Oncological & Neurological \\
\hline Vallecular pooling & 0 & $78(54)$ & $92(63)$ \\
& 1 & $47(32)$ & $39(27)$ \\
& 2 & $20(14)$ & $15(10)$ \\
\hline Pyriform sinus pooling & 0 & $128(75)$ & $131(74)$ \\
& 1 & $32(19)$ & $45(25)$ \\
& 2 & $10(5.9)$ & $1(0.6)$ \\
\hline Piecemeal deglutition & 0 & $26(15)$ & $39(22)$ \\
& 1 & $59(34)$ & $74(43)$ \\
& 2 & $36(21)$ & $32(18)$ \\
& 3 & $14(8.1)$ & $10(5.7)$ \\
& 4 & $37(22)$ & $19(11)$ \\
\hline
\end{tabular}

The scores of the observer with the highest intraobserver agreement level were used for the analysis

\section{Intraobserver agreement}

The level of intraobserver agreement ranged from 0.79 to 0.93 for observer 1 and from 0.76 to 0.90 for observer 2 (Table 5.3). The posterior distribution of the Bayesian nonlinear mixed model parameters for intraobserver agreement is summarized in Table 5.4. The level of intraobserver agreement was similar for both observers, with the exception of postswallow vallecular pooling: observer 1 had a higher intraobserver agreement than observer 2 on that variable. There was no difference in intraobserver agreement between oncological and neurological patients, nor between thin and thick liquid consistencies.

Table 5.3 Linear weighted kappa coefficient (SE) of agreement for all rating tasks

\begin{tabular}{|c|c|c|c|c|c|c|}
\hline \multirow[t]{2}{*}{ FEES variables } & \multicolumn{2}{|c|}{ Intraobserver agreement } & \multicolumn{2}{|c|}{ Interobserver agreement } & \multicolumn{2}{|c|}{ Intrapanelagreement } \\
\hline & Observer 1 & Observer 2 & Thin liquid & Thick liquid & Total & Total \\
\hline Piecemeal deglutition & $0.86(0.041)$ & $0.90(0.026)$ & $0.84(0.033)$ & $0.93(0.019)$ & $0.88(0.020)$ & $0.95(0.029)$ \\
\hline $\begin{array}{l}\text { Postswallow vallecular } \\
\text { pooling }\end{array}$ & $0.93(0.041)$ & $0.79(0.068)$ & $0.30(0.075)$ & $0.76(0.040)$ & $0.65(0.037)$ & $0.85(0.071)$ \\
\hline $\begin{array}{l}\text { Postswallow pyriform } \\
\text { sinus pooling }\end{array}$ & $0.79(0.054)$ & $0.76(0.084)$ & $0.55(0.071)$ & $0.67(0.069)$ & $0.61(0.059)$ & $0.91(0.068)$ \\
\hline $\begin{array}{l}\text { Laryngeal } \\
\text { penetration/tracheal } \\
\text { aspiration }\end{array}$ & $0.79(0.064)$ & $0.79(0.066)$ & $0.82(0.037)$ & $0.58(0.070)$ & $0.73(0.035)$ & $0.93(0.049)$ \\
\hline
\end{tabular}

SE standard error 


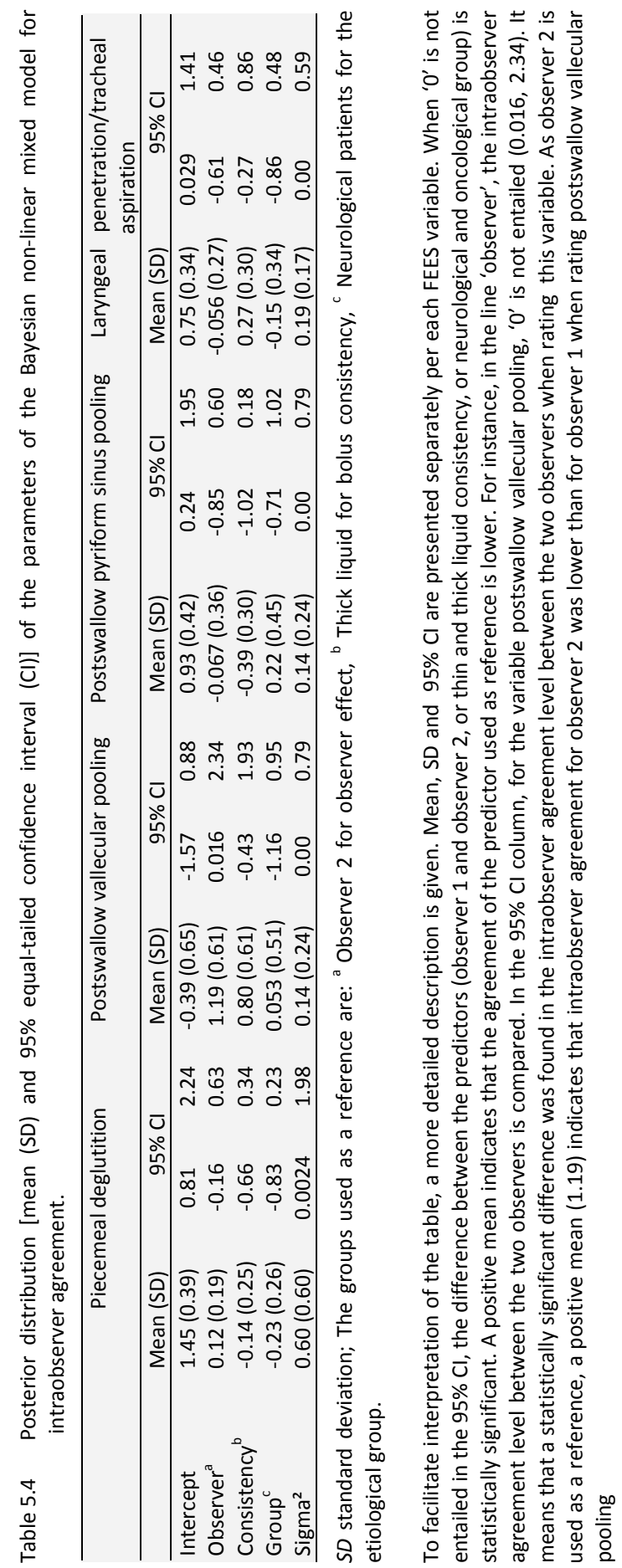




\section{Interobserver agreement}

Interobserver agreement levels are presented in Table 5.3 according to the bolus consistency. The posterior distribution of the Bayesian non-linear mixed model parameters for interobserver agreement is summarized in Table 5.5. Interobserver agreement was lower for thin liquid than for thick liquid swallow trials on the variables piecemeal deglutition and postswallow vallecular pooling. The opposite was observed for the measurements of the variable laryngeal penetration / tracheal aspiration. Interobserver agreement was slightly lower on the postswallow pyriform sinus pooling scale for thin liquid trials compared with thick liquid. On closer inspection, disagreement between the two observers occurred mainly at the first two levels of the scale (normal and mild impairment). There was no difference in the level of interobserver agreement for oncological versus neurological patients.

\section{Consensus panel agreement}

The intrapanel agreement level is presented in Table 5.3. Comparison of the scores given independently to those given on the consensus panel for exactly the same FEES measurement reveals that the magnitude of the changes in the score varies according to the FEES variable assessed. The probability that an independent score would change on the consensus panel was $27 \%$ for postswallow vallecular pooling, $17 \%$ for postswallow pyriform sinus pooling, $16 \%$ for piecemeal deglutition, and $14 \%$ for laryngeal penetration/tracheal aspiration. The frequency of such changes was slightly higher for the variable postswallow vallecular pooling during thick liquid swallows compared with thin liquid (Table 5.6). No statistically significant difference was detected in the frequency of changes in FEES measurements between etiological groups and between observers, with one exception: postswallow pyriform sinus pooling, where changes were more frequent for observer 1. 


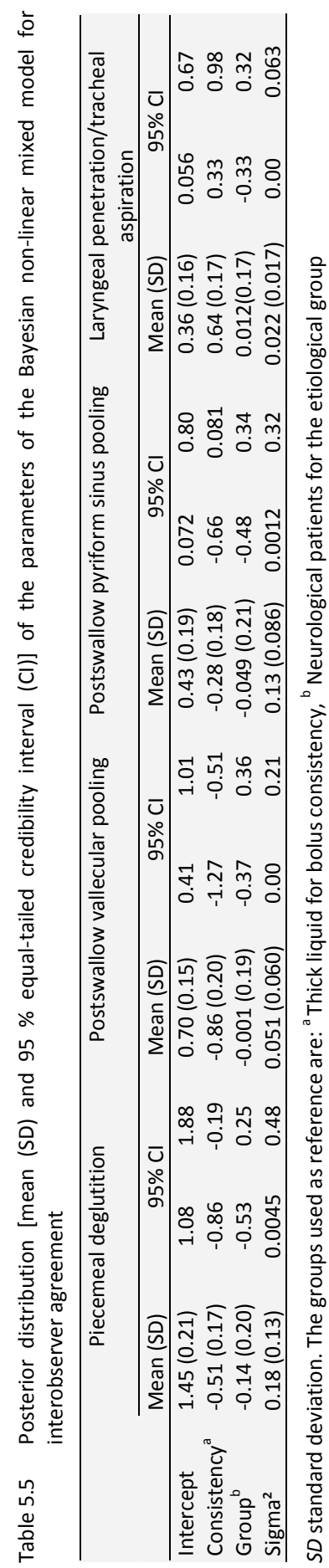

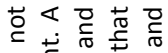
을 는 c

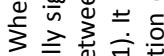
ऽ 늠 市 苋过

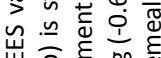

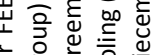
๙ั

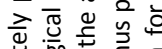
节产 总 О 讨

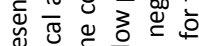

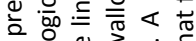
능 인 究 ธั

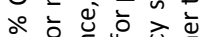
๙ ர்

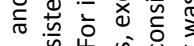

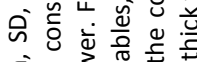
동 흘 으 은 둔 $\sum$ 흐 뜨 의

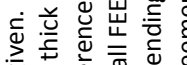

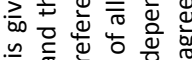
을 ๘

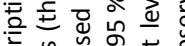
ปั

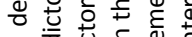

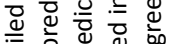

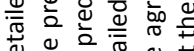

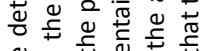
잉 ह ब

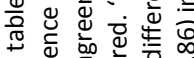

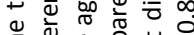

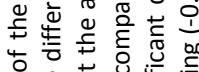

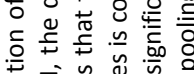

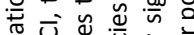

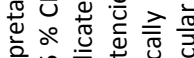
늉 $\stackrel{.}{\subseteq}$ d ఖ

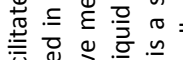

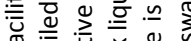

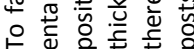




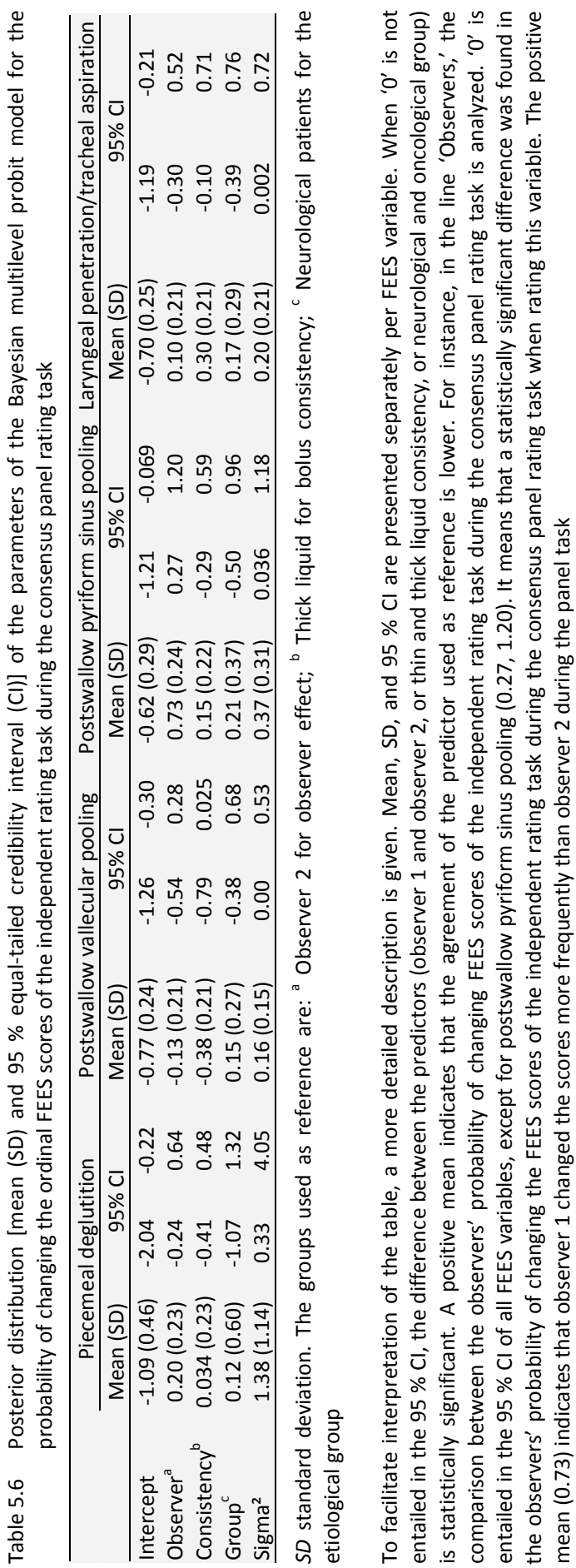




\section{Discussion}

The two main aspects of an outcome measurement are validity (how accurate are the measurements) and reproducibility (how similar are the results of the repeated measurements). Although both concepts are related, they can be investigated separately. Observers' agreement is the first step to show validity as it is not possible to have a valid scale if the measurements are not reproducible. The term reproducibility can be used to comprise two concepts, agreement and reliability, because both concepts concern the question of whether measurement results are reproducible in test-retest situations. Agreement parameters assess how close the results of the repeated measurements are, by estimating the measurement error in repeated measurements. Reliability parameters assess whether study objects, often persons, can be distinguished from each other, despite measurement errors. In that case, the measurement error is related to the variability between persons. Consequently, reliability parameters are highly dependent on the heterogeneity of the study sample, while the agreement parameters, based on measurement error, are more a pure characteristic of the measurement instrument. ${ }^{15}$ Therefore, the present study analyses intra- and interobserver agreement and explores any discrepancy in the ratings to better understand the causes of disagreement among observers. The effects of dysphagia etiology, different observers, and bolus consistency on the agreement levels were analyzed in two types of rating tasks: independent rating (intra- and interobserver agreement), and consensus panel rating (intrapanel observer agreement).

The effect of dysphagia etiology (neurological or head and neck oncological origin) on the agreement levels was also analyzed in all rating tasks. Except for aspiration where oncological patients presented higher scores, there was no effect of the dysphasia etiology on the other FEES variables. The absence of an effect of dysphagia etiology on agreement was unexpected, as it was presumed that alterations in the anatomy and physiology of the pharynx and/or larynx, secondary to cancer treatment, would influence the observers' agreement on the ratings. Apparently, the selected FEES variables are appropriate to evaluate both etiological groups. The results suggest that the training program offered sufficient information to enable the observers to evaluate swallowing function using FEES without taking changes in the anatomy and physiology of swallowing into account.

In the independent rating task, the intraobserver agreement level was similar for both observers, and there was no effect of bolus consistency. These findings show that the two observers had a similar interpretation of the ordinal scoring system and were consistent when repeating the measurements. In accordance with previous studies, intraobserver agreement was higher than agreement between the two observers (interobserver agreement). ${ }^{9,12}$

Overall, interobserver agreement levels were substantial $(k>0.61)$. However, a more detailed analysis demonstrated that agreement levels were affected by bolus consistency. For instance, during thin liquid trials, interobserver agreement for 
postswallow vallecular and pyriform sinus pooling was fair to moderate $(0.30$ and 0.55 , respectively). The lower interobserver agreement recorded for these measured variables concurs with findings reported elsewhere. ${ }^{12}$ Although bolus consistency is known to influence swallowing performance, the impact of consistency on observer agreement is underexplored. ${ }^{16,17}$

The lower levels of interobserver agreement might be explained as follows. First, even though the observers understood the ordinal scoring system well, as confirmed by the intraobserver agreement levels, they did not reach consensus on the cut-off points. The description of the rating scale does not give the precise range of each ordinal level, which leaves it up to the observers to set their own boundaries. Second, as thin liquid consistency is less cohesive, the bolus is not concentrated but instead spreads in the valleculae or pyriform sinus, thereby hindering an estimation of the amount of pooling. Moreover, the very nature of the FEES images makes it difficult to quantify precisely the amount of bolus left after swallowing., ${ }^{9,18}$

The intrapanel observer agreement levels were slightly higher than the intraobserver levels on the independent rating task. That difference suggests that consensus panel rating might offer an alternative to independent rating of FEES exams, as the discussion of cases in a panel may improve concordance. ${ }^{19}$ However, the agreement level obtained between two separate consensus panels with different members still needs to be explored, particularly in comparison to individual interobserver agreement levels.

Observers were consistent when re-scoring swallows independently or on the consensus panel. However, when repeating the task on the panel, they frequently adjusted the scores they had given previously when rating exactly the same measurements independently. That tendency to change in a panel setting reflects the observers' individual interpretation of the ordinal FEES scoring system. Furthermore, the probability of changing scores during the consensus panel rating task was similar for both observers. One explanation might be that, besides being inexperienced in rating FEES exams, the observers had followed the same intensive training program. Consensus panel ratings performed by observers with different levels of experience, or without specific training on FEES measurements, might yield other results.

\section{Limitations of the study}

The present study was based on FEES ratings of two observers. Comparing scores by a larger number of observers might produce different results. Furthermore, including students without experience in swallowing evaluation was a pre-experimental choice because we were interested in the agreement between naïve observers. Including more experienced observers might produce different results. The ordinal scales of the FEES outcome variables have been described in several previously published studies. ${ }^{12,17}$ However, they were not validated yet, which might have implications for the interpretation of the results. 


\section{Conclusion}

Observer agreement on measurements recorded in FEES exams was influenced by bolus consistency and not by dysphagia etiology, as defined in the present study design. It would be preferable to analyze observer agreement on FEES measurements according to bolus consistency, as this variable apparently affects the interpretation of the outcome. This study illustrates how the identification of factors that might influence agreement levels could elucidate the rating process. Investigations such as this one could assist in developing a more precise measurement scale to improve observer agreement on measurements in FEES exams. 


\section{References}

1. Langmore SE, Schatz K, Olsen N. Fiberoptic endoscopic examination of swallowing safety: a new procedure. Dysphagia. 1988;2(4):216-9.

2. Hiss SG1, Postma GN. Fiberoptic endoscopic evaluation of swallowing. Laryngoscope. 2003;113(8): 1386-93.

3. Hind JA, Gensler G, Brandt DK, Gardner PJ, Blumenthal L, et al. Comparison of trained clinician ratings with expert ratings of aspiration on videofluoroscopic images from a randomized clinical trial. Dysphagia. 2009;24(2):211-7.

4. Scott A, Perry A, Bench J. A study of inter reliability when using videofluoroscopy as an assessment of swallowing. Dysphagia. 1998;13(4):223-7.

5. Frowen JJ, Cotton SM, Perry AR. The stability, reliability, and validity of videofluoroscopy measures for patients with head and neck cancer. Dysphagia. 2008;23(4):348-63.

6. Baijens L, Barikroo A, Pilz W. Intrarater and interrater reliability for measurements in videofluoroscopy of swallowing. Eur J Radiol 2013;82(10):1683-95.

7. Rosenbek JC, Robbins JA, Roecker EB, Coyle JL, Wood JL. A penetration-aspiration scale. Dysphagia. 1996;11(2):93-8.

8. Colodny N. Interjudge and intrajudge reliabilities in fiberoptic endoscopic evaluation of swallowing (fees) using the penetration-aspiration scale: a replication study. Dysphagia. 2002;17(4):308-15.

9. Kelly AM1, Leslie P, Beale T, Payten C, Drinnan MJ. Fibreoptic endoscopic evaluation of swallowing and videofluoroscopy: does examination type influence perception of pharyngeal residue severity? Clin Otolaryngol. 2006;31(5):425-32.

10. Kelly AM, Drinnan MJ, Leslie P. Assessing penetration and aspiration: how do videofluoroscopy and fiberoptic endoscopic evaluation of swallowing compare? Laryngoscope. 2007;117(10):1723-7.

11. Vanbelle S, Mutsvari T, Declerck D, Lesaffre E. Hierarchical modelling of agreement. Stat Med. 2012; 31(28);3667-80.

12. Baijens LW1, Speyer R, Passos VL, Pilz W, van der Kruis J, Haarmans S, Desjardins-Rombouts C. Surface electrical stimulation in dysphagic Parkinson patients: a randomized clinical trial. Laryngoscope. 2013;123(11):38-44.

13. Landis JR, Koch GG. The measurement of observer agreement for categorical data. Biometrics. 1977;33:159-174.

14. Yang Z1, Zhou M. Weighted kappa statistic for clustered matched-pair ordinal data. Comput. Stat. Data Anal. 2015;82: 1-18

15. de Vet HC, Terwee CB, Knol DL, Bouter LM. When to use agreement versus reliability measures. J Clin Epidemiol. 2006;59(10):1033-9.

16. Steele CM, Alsanei WA, Ayanikalath S, Barbon CE, Chen J, et al. The Influence of Food Texture and Liquid Consistency Modification on Swallowing Physiology and Function: A Systematic Review. Dysphagia. 2015;30(1):2-26.

17. Pilz W, Baijens LW, Passos VL, et al. Swallowing assessment in myotonic dystrophy type 1 using fiberoptic endoscopic evaluation of swallowing (FEES). Neuromuscul Disord. 2014; 24(12):1054-62.

18. Farneti D, Fattori B, Nacci A, et al. The Pooling-score (P-score): inter- and intra-rater reliability in endoscopic assessment of the severity of dysphagia. Acta Otorhinolaryngol Ital. 2014;34(2):105-10.

19. Levine RD, Sugarman M, Schiller W, Weinshel S, Lehning EJ, Lagasse RS. The effect of group discussion on interrater reliability of structures peer review. Anesthesiology. 1998;89(2):507-15. 



\section{Chapter 6}

\section{Identifying patterns of FEES-derived swallowing trajectories using group-based trajectory model}

Laura W.J. Baijens

Walmari Pilz

Bernd Kremer

Valeria Lima Passos

Dysphagia. 2015;30(5):529-39 


\section{Abstract}

The present study delineates and visualizes swallowing trajectories along seven swallow trials in dysphagic patients using group-based trajectory modeling (GBTM). This model facilitates the recognition of swallowing functional categories, estimates their frequency of occurrence, and enhances the understanding of swallowing dynamics. Two hundred and five dysphagic patients underwent a standardized FEES examination protocol. Five ordinal variables were blindly assessed for each swallow by two observers independently. GBTM analysis was conducted to find and characterize trajectories of FEES responses. For most FEES outcome variables, trajectories were qualitatively distinct in degree and kind (level of impairment and how this changed over the seven swallow trials). Two FEES outcome variables - delayed initiation of the pharyngeal reflex and postswallow pyriform sinus pooling - showed the highest prevalence of severe swallowing impairment. Highly impaired categories were more stable throughout the different swallow trials. Intermediate trajectories, by contrast, were erratic, responding more sensitively to shifts in bolus consistency. GBTM can identify distinct developmental trajectories of measured FEES variables in patients with oropharyngeal dysphagia. In clinical practice, classification into distinct groups would help to identify the subgroup of dysphagic patients who may need specific medical attention. 


\section{Introduction}

Fiberoptic endoscopic evaluation of swallowing (FEES) is a reliable tool that allows the dysphagia professional to evaluate the pharyngeal phase of swallowing. ${ }^{1}$ FEES is well tolerated, easily repeatable, and can be performed at the bedside. ${ }^{1}$ During a standardized FEES examination, patients swallow a sequence of boluses of different consistencies in consecutive order. ${ }^{2,3}$ Usually visuoperceptual ordinal-scale variables are applied to judge FEES images.

FEES data on dysphagic patients are highly heterogeneous, both within and between subjects. This variability is partly due to the diversity of the dysphagic population, reflecting different etiologies of the swallowing dysfunction [Parkinson's disease (PD), stroke, myopathies, head and neck cancer (HNC), etc.]. However, even within a single etiological group, variability can be substantial. For instance, PD, which is characterized by progressive neurodegeneration, covers a large group of patients with different levels of disease severity. ${ }^{2}$ Therefore, acknowledging potential reasons for the high variability in FEES response (as recorded by repeated measures over the seven swallows) calls for a different analytical approach, one that capitalizes on the heterogeneity of these responses. In this respect, an alternative approach may shed new light on the dynamics of patients' swallowing capabilities. The alternative presented here allows discernible patterns of swallowing courses to be extracted from the FEES responses.

Group-based trajectory modeling (GBTM) was developed to identify groups of individuals following a similar progression of a certain behavior in a longitudinal setting. This model-based clustering method is often referred to as a person-centered approach. $^{4-6}$ It enables researchers to understand how life-course experiences unfold at the individual level and to cluster individuals who share similar developmental patterns. In GBTM, the population of interest is assumed to be heterogeneous - a mixture of groups. Shi et al. applied GBTM to describe the heterogeneity of symptom burden among patients with HNC and to identify subgroups with distinct symptom development trajectories. ${ }^{7}$ Treatment-related symptom burden varies significantly among patients undergoing radiotherapy or chemoradiotherapy, yet such variation is typically not reflected in the results from single-group studies. A two group GBTM model identified $68 \%$ of patients as having high symptom burden, associated with older age, worse baseline performance status, and chemoradiotherapy treatment. ${ }^{7}$ Another example of how GBTM has been used in the past is the study by Pines et al.. ${ }^{8}$ They described sexual risk trajectories among HIV-negative men who have sex with men. Three sexual risk trajectory groups were identified: low-risk, moderate-risk, and highrisk sexual behavior. The trajectories were significantly associated with earning an income, distress/depression symptoms, and substance use. ${ }^{8}$

However, a FEES examination protocol is of short duration, representing only a snapshot of a patient's swallowing functional state. Therefore, GBTM is used here primarily for exploratory purposes. The main objective was to identify subgroups of patients with qualitatively distinct responses over the seven swallowing trials. This 
would allow us to describe the swallowing trajectories' level, shape, and prevalence and then link these features to the etiology of dysphagia. To our knowledge, GBTM has not yet been considered for analysis of FEES data.

\section{Materials and methods}

\section{Participants}

Patients were consecutively enrolled in this prospective study while visiting the outpatient clinic of the Maastricht University Medical Center (MUMC) for their dysphagic complaints. Their data were collected as part of the regular healthcare program for oropharyngeal dysphagia (daily clinical practice). ${ }^{9}$ Incoming patients with oropharyngeal dysphagia could be divided into three main diagnostic groups. Dysphagia in the first group was due to HNC and possible oncological treatment effects on swallowing. Dysphagia in the second group was accompanied by PD. In the final group, it was due to myotonic dystrophy type 1 (DM1). Other etiologies of oropharyngeal dysphagia (stroke, Zenker diverticulum, cervical spine degeneration, etc.) were significantly underrepresented in the patient population over the enrollment period (2012-2014) and were not included. During the patient interview, all subjects reported subjective clinical complaints of oropharyngeal dysphagia ranging from mild to severe. These included, among others, slow eating due to prolonged bolus transit times, oropharyngeal passage disorder, coughing while drinking, and choking on foods. All patients were able to perform a swallow on command. The following exclusion criteria were applied: a Mini Mental State Examination (MMSE) score below $23^{10}$; concurrent HNC and a neurological disease (or neurosurgical brain intervention); head and neck oncological treatment less than 3 months previously; surgery of the head and neck swallowing region in patients with PD or DM1; extreme fatigue or weakness (unable to sit upright); an unstable period of PD (periods with large fluctuations, especially in motor function); not the same medication regimen for the past 6 weeks in neurological patients (e.g., with PD); and a total laryngectomy.

\section{FEES examination protocol}

All patients underwent a standardized examination protocol including a clinical otorhinolaryngological examination by a laryngologist, a clinical observation of oral intake by a speech and language pathologist, a FEES examination, and the MMSE. ${ }^{9,10}$ During the FEES examination, the participants were offered three trials of thin and three trials of thick liquid followed by one small bite-sized cracker (making a total of seven swallow trials). Each liquid trial contained $10 \mathrm{cc}$ of water or applesauce and was dyed with five percent methylene blue. The tip of the flexible fiberoptic endoscope Pentax FNL-10RP3 (Pentax Canada Inc., Mississauga, Ontario, Canada) was positioned 
just above the epiglottis in what is called the high position. ${ }^{1}$ FEES images were obtained using an Alphatron Stroboview ACLS camera, Alphatron light source, and IVACX computerized video archiving system (Alphatron Medical Systems, Rotterdam, The Netherlands) and recorded on a DVD. Neither a nasal vasoconstrictor nor a topical anesthetic had been administered to the nasal mucosa. All examinations were performed during the "on" motor phase in the PD patients (within 90-120 min after the intake of antiparkinsonian medication). ${ }^{11}$

\section{FEES outcome variables}

Visuoperceptual ordinal variables were scored for each swallow trial at varying speed (slow motion, normal, up to frame-by-frame speed) (Table 6.1). ${ }^{2,3,12}$

Table 6.1 Description and observer agreement levels for the measured FEES variables

\begin{tabular}{|c|c|c|c|}
\hline $\begin{array}{l}\text { FEES outcome } \\
\text { variable }\end{array}$ & Description & Scale $^{a}$ & Interobserver agreement $^{\mathrm{b}}$ \\
\hline $\begin{array}{l}\text { Piecemeal } \\
\text { deglutition }\end{array}$ & $\begin{array}{l}\text { Sequential swallowing } \\
\text { on the same bolus }\end{array}$ & $\begin{array}{l}\text { Five-point scale }(0-4) \\
0=\text { no additional swallows } \\
1=\text { one additional swallow } \\
2=\text { two additional swallows } \\
3=\text { three additional swallows } \\
4=\text { four or more additional swallows }\end{array}$ & $\begin{array}{l}\text { Almost perfect } \\
\text { agreement for all } \\
\text { etiologies (HNC, PD, } \\
\text { DM1) } \\
\text { Kappa 0.81-0.99 }\end{array}$ \\
\hline $\begin{array}{l}\text { Delayed initiation of } \\
\text { the pharyngeal } \\
\text { reflex }\end{array}$ & $\begin{array}{l}\text { Delayed onset of the } \\
\text { pharyngeal triggering }\end{array}$ & $\begin{array}{l}\text { Three-point scale }(0-2) \\
\begin{aligned} 0= & \text { no delay } \\
1= & \text { head of bolus in valleculae } \\
& \text { before initiation of pharyngeal } \\
& \text { reflex } \\
2= & \text { head of bolus in pyriform sinuses } \\
& \text { or lower before initiation of } \\
& \text { pharyngeal reflex }\end{aligned}\end{array}$ & $\begin{array}{l}\text { Substantial agreement } \\
\text { for PD and DM1 } \\
\text { Kappa } 0.61-0.80\end{array}$ \\
\hline $\begin{array}{l}\text { Postswallow } \\
\text { vallecular pooling }\end{array}$ & $\begin{array}{l}\text { Pooling in the } \\
\text { valleculae after the } \\
\text { swallow }\end{array}$ & $\begin{array}{l}\text { Three-point scale }(0-2) \\
0=\text { no pooling } \\
1=\text { filling of less than } 50 \% \text { of the } \\
\quad \text { valleculae } \\
2=\text { filling of more than } 50 \% \text { of the } \\
\quad \text { valleculae }\end{array}$ & $\begin{array}{l}\text { Almost perfect } \\
\text { agreement for all } \\
\text { etiologies (HNC, PD, } \\
\text { DM1) } \\
\text { Kappa 0.81-0.99 }\end{array}$ \\
\hline $\begin{array}{l}\text { Postswallow } \\
\text { pyriform sinus } \\
\text { pooling }\end{array}$ & $\begin{array}{l}\text { Pooling in the } \\
\text { pyriform sinuses after } \\
\text { the swallow }\end{array}$ & $\begin{array}{l}\text { Three-point scale }(0-2) \\
0=\text { no pooling } \\
1=\text { trace to moderate pooling } \\
2=\text { severe pooling up to complete } \\
\quad \text { filling of the sinuses }\end{array}$ & $\begin{array}{l}\text { Substantial agreement } \\
\text { for HNC and DM1 } \\
\text { Kappa } 0.61-0.80\end{array}$ \\
\hline $\begin{array}{l}\text { Penetration- } \\
\text { aspiration }\end{array}$ & $\begin{array}{l}\text { Penetration or } \\
\text { aspiration }\end{array}$ & $\begin{array}{l}\text { Three-point scale }(0-2) \\
0=\text { no penetration } \\
1=\text { penetration } \\
2=\text { aspiration }\end{array}$ & $\begin{array}{l}\text { Almost perfect } \\
\text { agreement for all } \\
\text { etiologies (HNC, PD, } \\
\text { DM1) } \\
\text { Kappa 0.81-0.99 }\end{array}$ \\
\hline
\end{tabular}

$<0$ less than chance agreement; 0.01-0.20 slight agreement; 0.21-0.40 fair agreement; 0.41-0.60 moderate agreement; 0.61-0.80 substantial agreement; 0.81-0.99 almost perfect agreement; HNC head and neck cancer, $P D$ Parkinson's disease, DM1 myotonic dystrophy type $1 ;{ }^{a}$ Lower scores refer to normal functioning whereas higher scores refer to more severe disability; ${ }^{b}$ Kappa agreement (linear weighted kappa coefficient) 
Before assessment of the swallowing acts, two experts received consensus training for these ordinal variables. The protocol of this training has been described in previous studies. $^{2,3,12}$ The judges were blinded to the etiological group and to each other's ratings (independent rating). The swallow trials of all participants were scored in randomized order. To obtain intra-observer agreement, each observer performed repeated measurements (again blinded) of all visuoperceptual FEES variables during the second and third swallow of each bolus consistency for twenty randomly selected participants. This was done within a period of 2 weeks. Furthermore, observers were advised to limit the duration of the measurement sessions (max. $2 \mathrm{~h}$ per session) to avoid fatigue and prevent instability of observers' characteristics.

\section{Statistical analyses}

\section{Descriptive statistics}

Clinical and demographic variables are presented as means and standard deviation (SD) for continuous scales or as absolute numbers and proportions for categorical scales for each etiological category. Comparisons among etiological groups (HNC, PD, and DM1) were conducted with independent samples $t$ test or Chi square/Fisher's exact tests. Intra- and interobserver agreement was calculated using a linear weighted kappa coefficient.

\section{Group-Based Trajectory Modeling (GBTM)}

GBTM analysis was conducted to find and characterize swallowing courses (trajectories) for each measured FEES variable. Once such trajectories are identified, they can be described in terms of composition (size of the cluster), level of impairment (e.g., high, low), and how the impairment changes over the swallows (shape and nature of change: stable, erratic, improving, deteriorating, etc.). Given the way the FEES protocol is designed, with a fixed order of bolus consistencies, it is important to keep in mind that the interpretation of the trajectory's shape indicates how the swallowing function may be affected by the bolus consistencies.

\section{Model fitting, selection, and adequacy}

The measured FEES variables were analyzed with 'proc traj,' an SAS macro developed by Nagin et al.." ${ }^{4-6}$ All outcomes with three categories (three-point-scale ordinal variables) were dichotomized prior to statistical analysis. Categories ' 1 ' and ' 2 ' were changed into a new category, ' $1+$,' indicating impaired swallowing. The category ' 0 ' was unchanged and represented normal swallowing. For the variable penetrationaspiration, dichotomization of the data was carried out by collapsing normal and penetration into category ' 0 .' Aspiration was represented by category ' $1+$ '. The outcome with five categories (five-point-scale ordinal variable piecemeal deglutition), though being ordinal at the manifest level, was analyzed as a continuous variable. With 
proc traj, the trajectories' levels and shapes are determined by the model's regression parameters. Specifically, each latent trajectory can be characterized by a starting value of impairment level (intercept) and possibly by a polynomial function (linear, quadratic, cubic), thereby capturing the start level and the shape of the developmental course, respectively. The parameters were estimated by maximum likelihood, with the link functions 'logit' for dichotomized FEES variables and 'censored normal' for piecemeal deglutition, respectively.

Given the relatively small sample size, the maximum number of clusters considered was five $(k=5)$ and the highest polynomial order was cubic. Model fitting was conditioned on the risk factor etiology. Model selection was conducted in a two-step procedure, according to recommended guidelines. ${ }^{4-6}$ Starting with the highest number of clusters $(k=5)$, each with a cubic polynomial trend, $k$ was decreased until a model with the best Bayesian Information Criterion (smallest BIC) was obtained. Polynomial regression coefficients were selected on the grounds of statistical significance of the polynomial terms and BIC. Individuals' cluster assignment was based on the maximum posterior probability (PP) of cluster membership, as estimated by the selected model. Model adequacy was checked by means of the average posterior probability (APP) of each cluster (desirable average APP 0.7).

\section{Results}

\section{Observers' agreement}

The intra-observer agreement was sufficient for all FEES variables (kappa $C \geq 0.61$ ). For some FEES variables (delayed initiation of the pharyngeal reflex and postswallow pyriform sinus pooling), however, the interobserver agreement was insufficient when calculated per etiological subgroup (HNC, PD, DM1) (Table 6.1).

\section{Demographic and clinical characteristics}

Demographic and clinical characteristics are presented in Table 6.2 for each etiological subgroup. Two hundred and five patients were enrolled in this study. The flow diagram (Figure 6.1) displays the distribution of patients over the swallow trials according to their dysphagia etiology. Not everyone could complete all trials at each consistency, so some values are missing due to various causes. For example, HNC patients with severe xerostomia or without a dental prosthesis were not able to perform trials with a dry bite-sized cracker, so these values are reported as missing. Other patients exhibited more severe aspiration with thin liquids, resulting in less than three swallow trials for this consistency. Several DM1 patients performed six swallow trials but did not complete the sequence due to fatigue. It should be noted that data were obtained for all etiological groups (HNC, PD, and DM1: N=205) for piecemeal deglutition, 
postswallow vallecular pooling, and penetration-aspiration. For delayed initiation of the pharyngeal reflex and postswallow pyriform sinus pooling, the sample sizes were reduced to 116 (HNC and DM1) and 132 subjects (PD and DM1), respectively. The interobserver agreement for these measured variables was not sufficient for all etiological groups (Table 6.1).
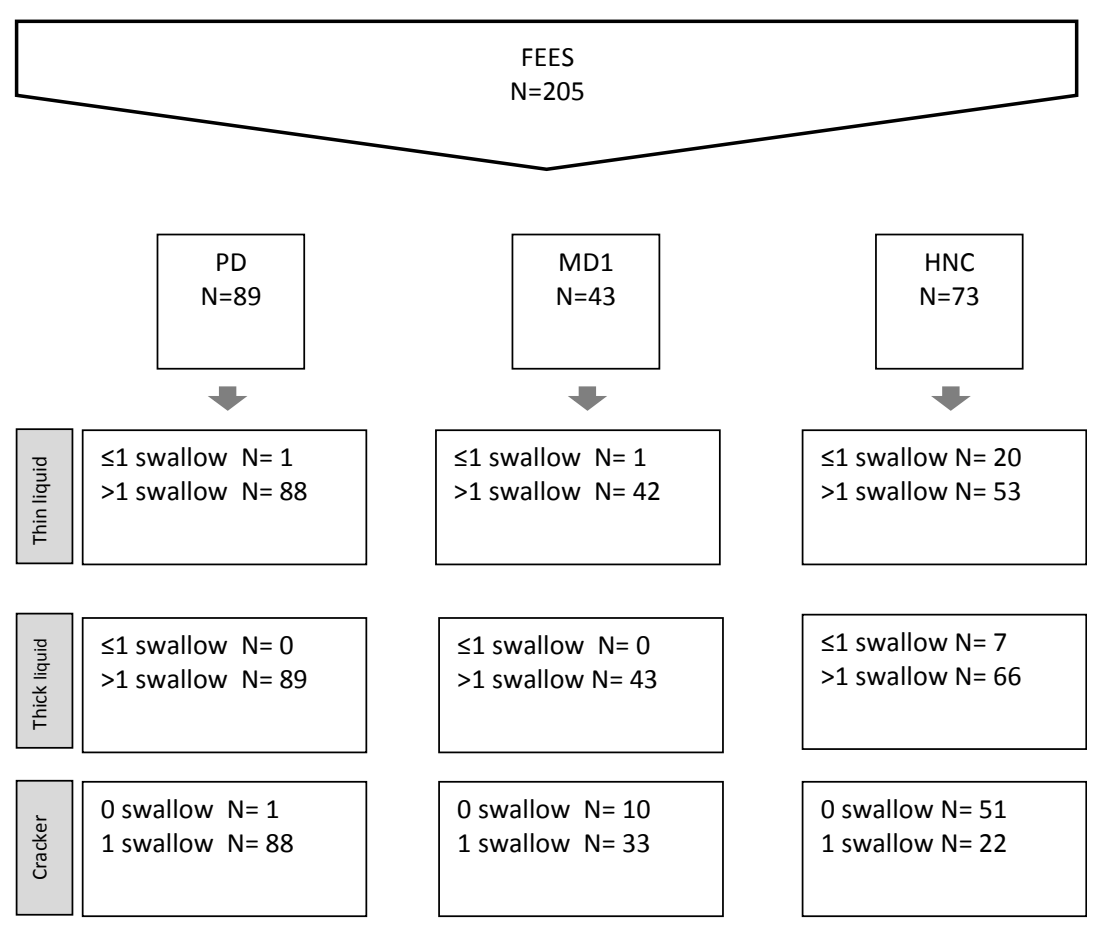

Figure 6.1 Flowchart of the number and etiology of the included dysphagic patients and the number of swallow trials per patient group and per consistency $\mathrm{HNC}=$ head and neck cancer; PD=Parkinson's disease; DM1=myotonic dystrophy type 1; FEES=fiberoptic endoscopic evaluation of swallowing

\section{GBTM Results}

Figures 6.2, 6.3, 6.4, 6.5 and 6.6, which illustrate the GBTM output, display the estimated level and shape of the identified FEES trajectories. They show how the probability of being classified in impaired categories (equal to $1+$ ) changed over the seven swallow trials. For the variable piecemeal deglutition, changes in averages over the swallow trials are displayed instead of probabilities (Figure 6.2). 
Table 6.2 Patients' demographic and clinical characteristics

\begin{tabular}{|c|c|c|c|c|c|c|}
\hline & $\begin{array}{c}\text { HNC } \\
(\mathrm{N}=73)\end{array}$ & & $\begin{array}{c}P D \\
(N=89)\end{array}$ & & $\begin{array}{c}\text { DM1 } \\
(\mathrm{N}=43)\end{array}$ & \\
\hline Male & 59 & & 66 & & 27 & \\
\hline \multirow[t]{2}{*}{ Female } & 14 & & 23 & & 16 & \\
\hline & Median $\left(25^{\text {th }}, 75^{\text {th }}\right.$ perc $)$ & Range & Median $\left(25^{\text {th }}, 75^{\text {th }}\right.$ perc $)$ & Range & Median $\left(25^{\text {th }}, 75^{\text {th }}\right.$ perc $)$ & Range \\
\hline Age & $67(60,73)$ & $30-83$ & $67(61,73)$ & $42-88$ & $46(38,55)$ & $21-69$ \\
\hline
\end{tabular}

HNC= head and neck cancer; PD= Parkinson's disease; DM1= myotonic dystrophy type 1, perc percentile
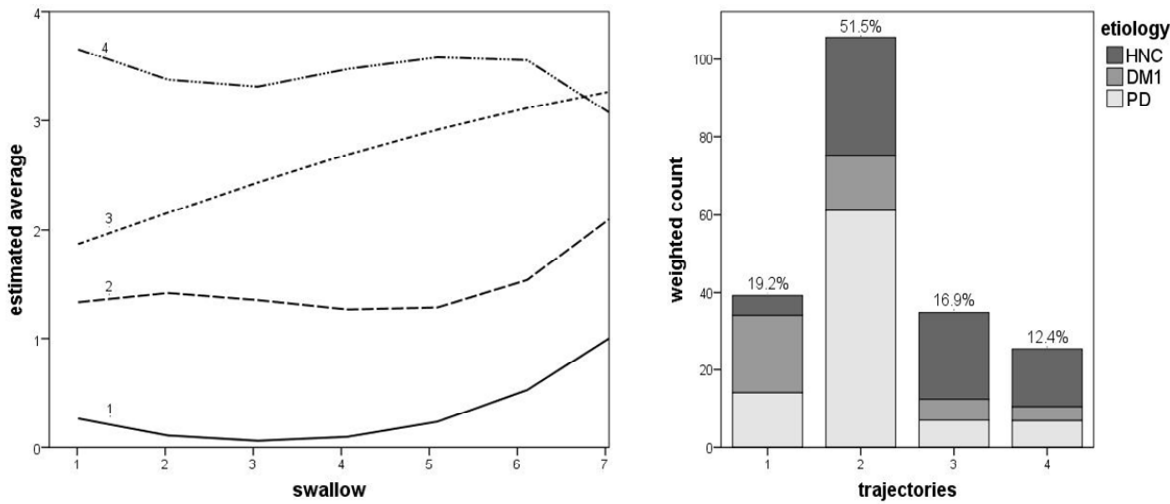

Figure 6.2 Four swallow trajectories of the FEES variable piecemeal deglutition (1 low impairment, 2-3 intermediate impairment, 4 high impairment). The $y$ axis represents the estimated average score. The $x$ axis represents the number of swallow trials and their consistency (1-3 thin liquid, 4-6 thick liquid, 7 bite-sized cracker) (left figure). The bar charts (in the right figure) present the estimated prevalence (\%) of the swallowing trajectories together with their etiology distribution

The mixture proportions (in \%, representing the estimated size of the latent cluster) are given together with the etiology distribution per trajectory (bar charts). There, the height of the bar indicates the count of subjects assigned to the trajectory, weighted by their respective posterior probabilities.

A great diversity in swallowing behavior was observed for all measured FEES variables. For most of them, the trajectories were qualitatively distinct, both in degree and kind. In general, the bottom and top FEES trajectories (usually 1 and 3 or 4), reflecting low and high impairment in swallowing function, were less erratic than the intermediate ones and thus less sensitive to changes in bolus consistency. These trajectories for all measured FEES variables can be seen as 'lines' of pulled data of all the patients, regardless of their diagnosis (HNC, PD, and DM1).

For piecemeal deglutition (Figure 6.2), four trajectories were identified. They were rather stable (flat), differing more in level of impairment than in their shapes/courses. The exception was trajectory no. 3, which steadily deteriorated over the swallows. In general, the swallowing function is more impaired with a bite-sized cracker. The etiology distributions over the trajectories are given in the bar charts of Figure 6.2. 
Relative to the bottom trajectory (1) and taking the PD group as a reference, HNC patients were more frequently assigned to the impaired trajectories $3\left(\mathrm{OR}_{\mathrm{HNC}}=8.41\right.$ with $95 \% \mathrm{Cl}[1.62,43.65])$ and $4\left(\mathrm{OR}_{\mathrm{HNC}}=5.75\right.$ with $\left.95 \% \mathrm{Cl}[1.10,29.85]\right)$. DM1 patients, by contrast, tended to cluster around the lower trajectories, being significantly less likely than PD patients to fall in trajectory $2\left(\mathrm{OR}_{\mathrm{DM} 1}=0.16\right.$ with $\left.95 \% \mathrm{Cl}[0.05,0.47]\right)$.

For one variable, delayed initiation of the pharyngeal reflex (PD and DM1, only), swallowing function improved as bolus consistency changed into thick liquid and bitesized cracker, either gradually (trajectory no. 3) or abruptly (trajectory no. 2) (Figure 6.3). For the cluster of patients in trajectory no. 1, swallowing function decreased as bolus consistency changed into thick liquid (Figure 6.3). Relatively many patients were assigned to the most impaired category (bar chart Figure 6.3). Etiologies were similarly distributed over the three trajectories.

In Figure 6.4 (postswallow vallecular pooling), the bottom trajectory (low impairment) had the highest prevalence (36.2\%). Note that for these patients, a slow and gradual deterioration was observed over the swallow trials. The intermediate trajectory no. 3 fared badly with liquids (high probability of impairment) and abruptly changed for the better with the swallow of a bite-sized cracker. Patients assigned to trajectory no. 2 showed the opposite behavior, deteriorating considerably as the bolus consistency changed into thick liquid and a bite-sized cracker. DM1 patients had significantly more chance to be assigned to the most stable and highly impaired trajectory no. 4 compared to $\mathrm{PD}$ subjects $\left(\mathrm{OR}_{\mathrm{DM} 1}=15.79\right.$ with $\left.95 \% \mathrm{Cl}[4.41,56.48]\right)$ (Figure 6.4).
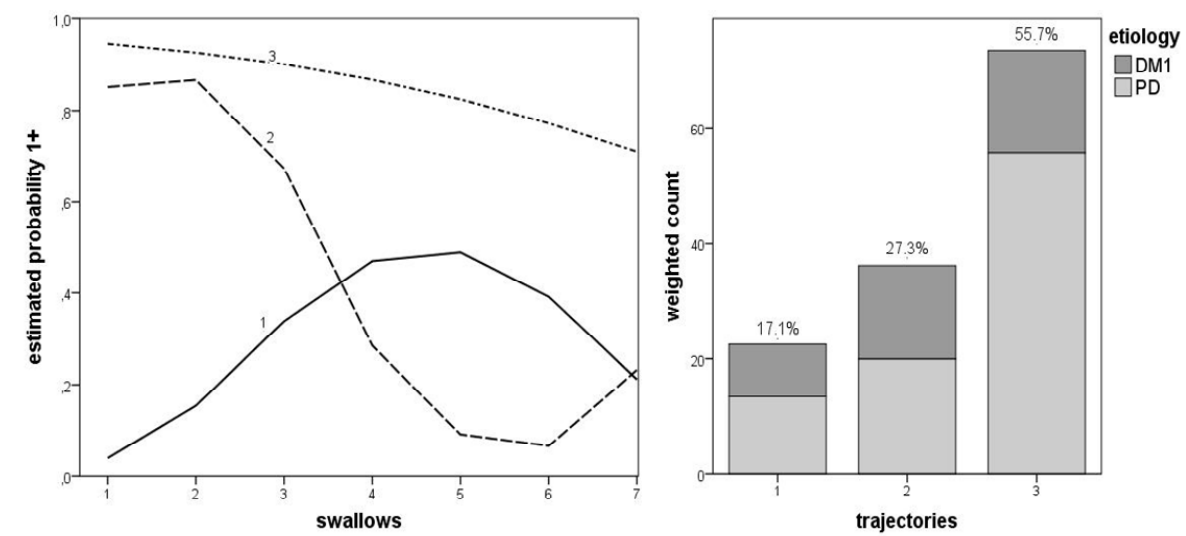

Figure 6.3 Three swallow trajectories of the FEES variable delayed initiation of the pharyngeal reflex ( 1 low impairment, 2 intermediate impairment, 3 high impairment). The $y$ axis represents the estimated probability of being classified in impaired categories (equal to $1+$ ). The $x$ axis represents the number of swallow trials and their consistency (1-3 thin liquid, 4-6 thick liquid, 7 bite-sized cracker) (left figure). The bar charts (in the right figure) present the estimated prevalence (\%) of the swallowing trajectories together with their etiology distribution. Note: The height of the bar indicates the number of patients, weighted by their posterior probability of assignment to the respective trajectory 
For the variable postswallow pyriform sinus pooling (DM1 and HNC only), the bottom trajectory, with the lowest impairment, had the highest prevalence (40.3\%) (Figure 6.5). The intermediate trajectory no. 2 abruptly changed for the worse when thick liquid swallow trials were given, with a minor recovery during ingestion of a bite-sized cracker. HNC patients had significantly less chance to be assigned to the highly impaired trajectory no. 3 compared with $\mathrm{DM} 1$ patients $\left(\mathrm{OR}_{\mathrm{DM} 1}=0.02\right.$ with $\left.95 \% \mathrm{Cl}[0.005,0.11]\right)$ (Figure 6.5).

The vast majority of patients ( $66 \%$ ) had a low probability of aspiration over all swallow trials (Figure 6.6). The rest were distributed over two distinct patterns of aspiration (trajectories 2 and 3). Patients assigned to trajectory no. 2 only showed a high probability of aspiration with thin liquid, their performance clearly improving with more solid consistencies. Patients in trajectory no. 3, by contrast, were clearly susceptible to aspiration, improving slightly with bite-size crackers. Etiology distributions differed significantly among the trajectories. DM1 and HNC patients were more likely to be assigned to trajectory no. 2 compared to $\mathrm{PD}$ patients, with $\mathrm{OR}_{\mathrm{DM} 1}=8.08,95 \% \mathrm{Cl}[1.45$, 44.92] and $\mathrm{OR}_{\mathrm{HNC}}=11.59,95 \% \mathrm{Cl}[2.17,61.79]$. The $\mathrm{ORs}$ for trajectory no. 3 were $\mathrm{OR}_{\mathrm{DM} 1}=3.56,95 \% \mathrm{Cl}[1.03,12.19]$ and $\mathrm{OR}_{\mathrm{HNC}}=12.93,95 \% \mathrm{Cl}[4.71,35.49]$.
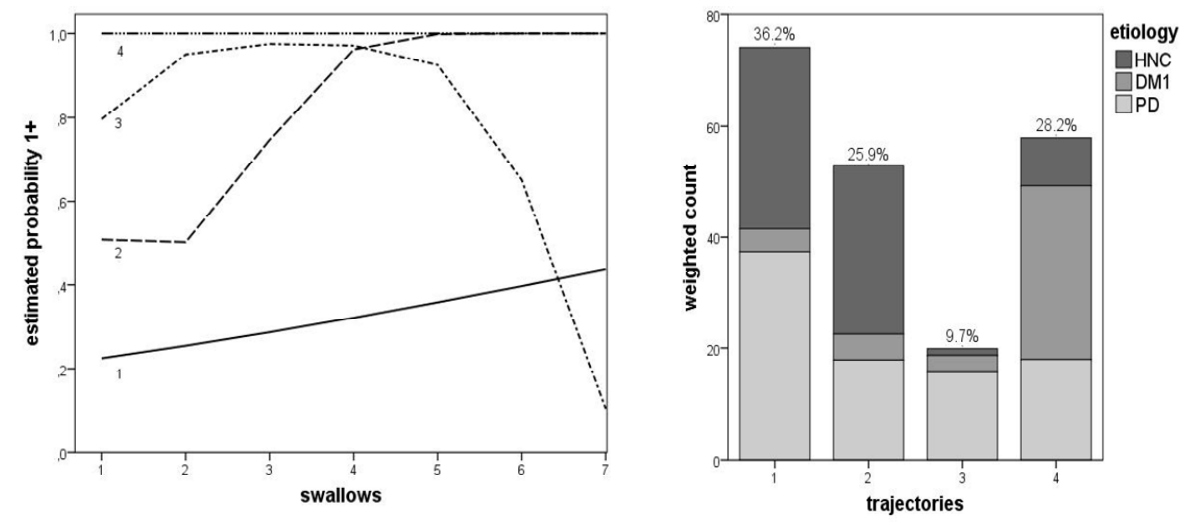

Figure 6.4 Four swallow trajectories of the FEES variable postswallow vallecular pooling (1 low impairment, 2-3 intermediate impairment, 4 high impairment). The $y$ axis represents the estimated probability of being classified in impaired categories (equal to $1+$ ). The $x$ axis represents the number of swallow trials and their consistency (1-3 thin liquid, 4-6 thick liquid, 7 bite-sized cracker) (left figure). The bar charts (in the right figure) present the estimated prevalence (\%) of the swallowing trajectories together with their etiology distribution 

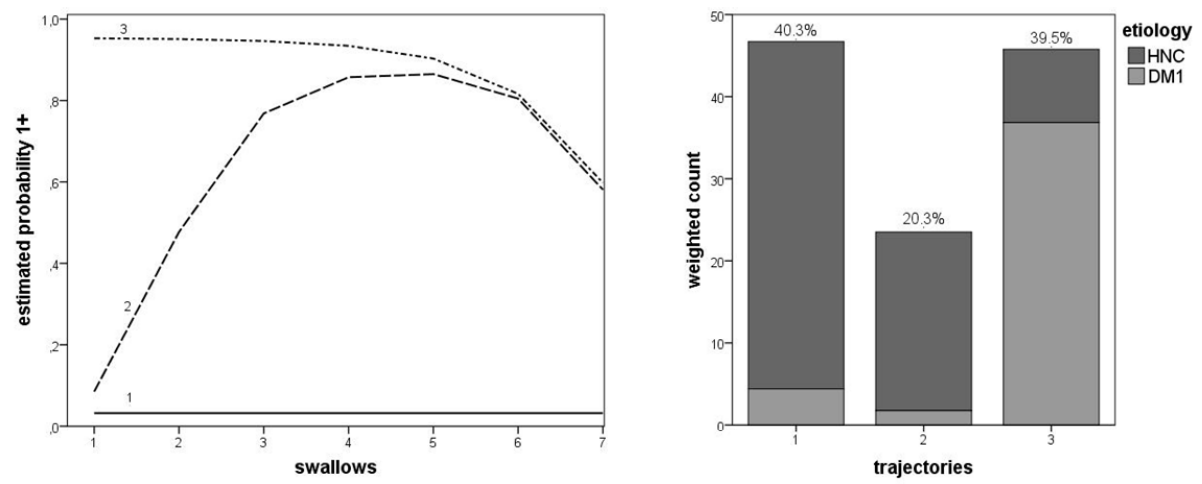

Figure 6.5 Three swallow trajectories of the FEES variable postswallow pyriform sinus pooling ( 1 low impairment, 2 intermediate impairment, 3 high impairment). The $y$ axis represents the estimated probability of being classified in impaired categories (equal to $1+$ ). The $x$ axis represents the number of swallow trials and their consistency (1-3 thin liquid, 4-6 thick liquid, 7 bite-sized cracker) (left figure). The bar charts (in the right figure) present the estimated prevalence $(\%)$ of the swallowing trajectories together with their etiology distribution
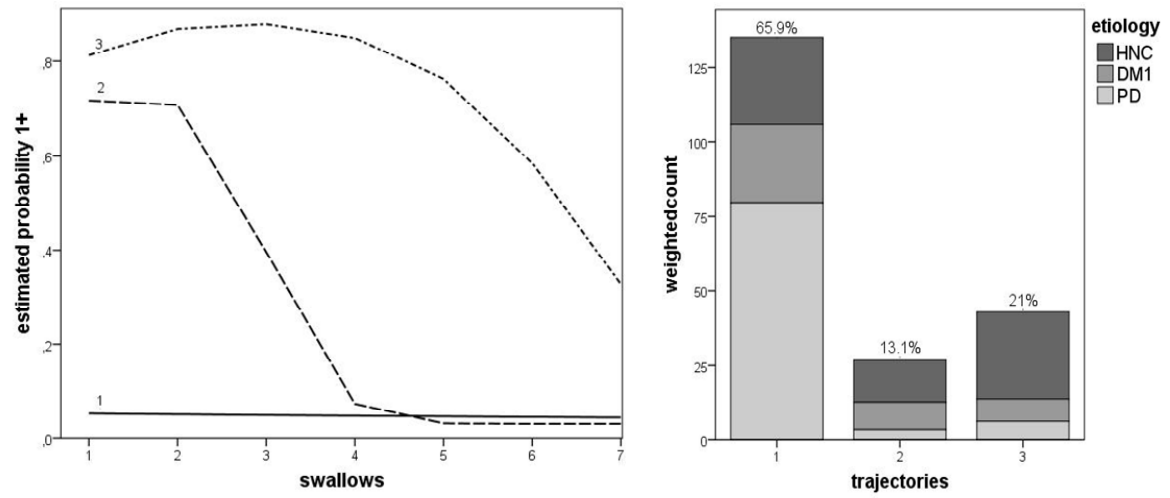

Figure 6.6 Three swallow trajectories of the FEES variable penetration-aspiration (1 low impairment, 2 intermediate impairment, 3 high impairment). The $y$ axis represents the estimated probability of being classified in impaired categories (equal to $1+$ ). The $x$ axis represents the number of swallow trials and their consistency (1-3 thin liquid, 4-6 thick liquid, 7 bite-sized cracker) (left figure). The bar charts (in the right figure) present the estimated prevalence (\%) of the swallowing trajectories together with their etiology distribution

\section{Discussion}

Heterogeneity of FEES outcomes makes it difficult for practitioners to pinpoint and characterize regular or irregular patterns of swallowing behavior in population-based studies. There is great demand for evidence-based data that could enhance the 
clinicians' interpretation, justify generalizations, and support potential clinical decisions.

This study confirms the hypothesis that GBTM can identify (latent) subgroups of developmental courses for measured FEES variables. Its application uncovered the variability of onset and demonstrated the reactive developmental changes as a function of the swallow trials. This information, in turn, facilitated the recognition of typical or atypical swallowing behaviors, allowing an estimation of their prevalence. The link of the trajectories to the etiology of oropharyngeal dysphagia was found to be significant for most FEES variables. For instance, DM1 patients tend to fall in the higher, impaired trajectories for the variables postswallow vallecular pooling and postswallow pyriform sinus pooling, whereas the opposite applies to the variables piecemeal deglutition and penetration-aspiration. ${ }^{3}$ This finding is consistent with a previous study showing more severe impairment for postswallow vallecular pooling when swallowing thick liquid compared with thin. DM1 patients performed better (i.e., had less impaired FEES outcomes) with the solid bolus consistency (bite-sized cracker) than with the other consistencies for the variable postswallow vallecular pooling. ${ }^{3}$

HNC patients, by contrast, fell in less impaired trajectories for the variables postswallow vallecular pooling and postswallow pyriform sinus. They had a higher risk of being assigned to top, impaired trajectories for the variables piecemeal deglutition and penetration-aspiration compared to DM1 patients., ${ }^{3,13}$ This makes sense, as (chemo) radiation therapy for HNC can cause sensory impairment, xerostomia, and fibrosis of the upper aerodigestive tract, any of which could increase the risk of aspiration and oral residue. $^{14}$

Furthermore, PD patients fell in higher impaired trajectories for the variable delayed initiation of the pharyngeal reflex. ${ }^{2}$ That finding was not unexpected; similar results have been observed in previous studies using this variable. ${ }^{2}$ Various motor disorders of PD have considerable influence on swallowing. For example, disturbed motility in the oral phase of swallowing is characteristic of PD. ${ }^{15,16}$ According to Nilsson et al., the prolongation of the oral-pharyngeal transit time is likely to reflect dysfunction caused by rigidity, bradykinesia (slowness of movement), and hypokinesia. ${ }^{17}$ This explanation concurs with the higher impaired trajectories we found for the variable delayed initiation of the pharyngeal reflex in PD. For the other measured FEES variables, PD patients were diversely distributed over the trajectories.

The application of GBTM in an analysis of FEES data is unprecedented, calling for some caution when weighing any new insights it might yield. Under the present circumstances and given the limitations of this study, our conclusions are preliminary and merely indicative. Nonetheless, a few of the interpretations enabled by trajectory clustering are noteworthy, as set forth below.

First, our results lend credence to the hypothesis that to use the 'one model fits all' approach, namely to model population trends, leads to an oversimplification of how patients react to the swallow trials. Such techniques may obscure deviations from the norm that are not necessarily linked to known clinical variables. Moreover, analysis of 
FEES data is often static, focusing on each bolus separately. Note, however, that, if we were to consider each one individually, the detected latent groups would be no longer separable. In our study, trajectories overlapped to a greater or lesser extent during certain parts of the examination. It was only from the developmental perspective that the differences became apparent and here lies the strength of GBTM. The visualization of functional groups may hint at diversity in the nature of change. For instance, for postswallow vallecular pooling, both trajectory nos. 2 and 3 can be considered as lying at an intermediate level of impairment and as being less stable than the other two extremes (upper and bottom trajectories). However, nos. 2 and 3 describe opposite swallowing behaviors. While cluster 2 swallowed better with thin liquids, the transition to thick liquids and a bite-sized cracker was accompanied by a deterioration of the swallowing function. In cluster 3 , by contrast, the deglutition of thick liquids and a bitesized cracker induced substantial improvement in swallowing response of the patients. It is unclear whether these findings reflect different underlying pathophysiological mechanisms affecting the swallowing response (for instance, if trajectory no. 2 would be measuring the effect of fatigue or weakness in the patient or an effect of post radiation xerostomia instead of bolus consistency, contrary to no. 3). ${ }^{4,13}$ Trajectory no. 2 applied to a larger proportion of HNC patients (post radiation xerostomia). The improvement described by trajectory no. 3 was observed mainly among PD patients and concerned postswallow vallecular pooling with the ingestion of a bite-sized cracker. The DM1 patients fell mainly in the severe impairment trajectory no. 4.

Second, any observed changes in trajectories were most likely induced by the consistency, since points of inflection often coincided with the transitions in the bolus sequence. However, other explanations cannot be disregarded. For instance, the intermediary trajectory no. 2 of postswallow pyriform sinus pooling shows that patients' swallowing function consistently deteriorated over the swallows, almost independently of the bolus consistencies. Apparently, the subjects fatigued quickly or suffered from severe post radiation xerostomia, leading to a more impaired swallowing function for thick liquid and a bite-sized cracker (mainly HNC patients in trajectory no. 2). Given these results, researchers might do well to adopt a more dynamic approach when analyzing and interpreting the various processes representing patients' reactive changes to a swallowing challenge. It is only by taking a developmental perspective that patients' dysfunctional behavior can be better discriminated and the pathophysiology of swallowing impairment properly understood.

The usefulness of GBTM for the analysis of FEES data goes beyond the visualization and characterization of the latent functional groups. Its impact greatly depends on the interpretational value of the trajectories and their theoretical plausibility. As argued here, GBTM provides an insightful depiction of swallowing behaviors. This conclusion needs to be explored further and replicated in larger-scale studies. Clinicians would then have a more detailed evaluation at their disposal, which they could use to support and guide their choices of rehabilitation programs or interventions. 


\section{Limitations of the study}

The present prospective study has some methodological limitations. First, its relatively small sample size posed a major constraint on data analysis with GBTM, as this technique requires larger samples to properly extract latent clusters. Nonetheless, the models converged without difficulty and the indices of model adequacy remained within acceptable bounds. For the analysis of three-point-scale ordinal FEES variables, dichotomization of the measured data may have over-simplified the overall swallow functioning of the patients due to loss of information. Another limitation concerns a well-known misunderstanding linked to GBTM analysis, the fallacy of reification. It occurs once latent trajectories are interpreted as real distinct entities. This should be avoided. The intent behind using GBTM was to describe the heterogeneity of FEES outcomes in such a way as to facilitate their clinical interpretation. The identified trajectories are not meant to represent definite classes of swallowing capabilities. The applied FEES protocol in the current study is the standardized protocol we use in daily clinical practice for many years. However, another FEES protocol might produce different results in capturing swallowing trajectories. Finally, a potential drawback of this study is that healthy controls were not included. However, the observed developmental trajectories as described above revealed the presence of clinically relevant subgroups of dysphagic patients in the study population.

\section{Conclusion}

GBTM identified distinct developmental trajectories of FEES responses, capturing the heterogeneity of swallowing function in patients with oropharyngeal dysphagia. These patterns could be linked to the underlying etiology of dysphagia. In clinical practice, such classification of patients into groups may help identify which patients need specific medical attention. GBTM is useful for describing the heterogeneity of dysphagia courses, for identifying groups to determine demographic or biological risk factors, and potentially for informing clinicians about subgroups of dysphagic patients who will need more attention to improve symptom control and quality of life. 


\section{References}

1. Langmore SE, Aviv JE. Endoscopic evaluation and treatment of swallowing disorders. New York: Thieme; 2001.

2. Baijens LW, Speyer R, Passos VL, Pilz W, van der Kuis J, Haarmans S, Desjardins-Rombouts C. Surface electrical stimulation in dysphagic Parkinson patients: a randomized clinical trial. Laryngoscope. 2013;123:E38-44.

3. Pilz W, Baijens LW, Passos VL, Verdonschot R, Wesseling F, Roodenburg N, Faber CG, Kremer B. Swallowing assessment in myotonic dystrophy type 1 using fiberoptic endoscopic evaluation of swallowing (FEES). Neuromuscul Disord. 2014;24:1054-62.

4. Nagin, D. S. (2005). Group-based modeling of development. Cambridge, Massachusetts: Harvard University Press.

5. Nagin DS, Odgers CL. Group-based trajectory modeling in clinical research. Annu Rev Clin Psychol. 2010;6:109-38.

6. Nagin DS, Tremblay RE. Analyzing developmental trajectories of distinct but related behaviors: a groupbased method. Psychol Methods. 2001;6:18-34.

7. Shi Q, Mendoza TR, Gunn GB, Wang XS, Rosenthal DI, Cleeland CS. Using group-based trajectory modeling to examine heterogeneity of symptom burden in patients with head and neck cancer undergoing aggressive non-surgical therapy. Qual Life Res. 2013;22:2331-9.

8. Pines HA, Gorbach PM, Weiss RE, Shoptaw S, Landovitz RJ, Javanbakht M, Ostrow DG, Stall RD, Plankey M. Sexual risk trajectories among MSM in the United States: implications for pre-exposure prophylaxis delivery. J Acquir Immune Defic Syndr. 2014;15:579-86.

9. Baijens LWJ. Multidisciplinaire polikliniek voor dysfagie. Nederlands Tijdschrift voor Keel-NeusOorheelkunde 18e jaargangnummer 1 januari 2012 p5-6.

10. Folstein MF, Folstein SE, McHugh PR. "Mini-mental state”. A practical method for grading the cognitive state of patients for the clinician. J Psychiatr Res. 1975;12:189-98.

11. Wajsbort J. The "off-on" phenomenon during treatment of Parkinson's disease with Levodopa. J Neurol. 1997;215:59-66.

12. Baijens LW, Speyer R, Pilz W, Roodenburg N. FEES Protocol Derived Estimates of Sensitivity: Aspiration in Dysphagic Patients. Dysphagia. 2014;29(5):583-90.

13. Kraaijenga SA, van der Molen L, Jacobi I, Hamming-Vrieze O, Hilgers FJ, van den Brekel MW. Prospective clinical study on long-term swallowing function and voice quality in advanced head and neck cancer patients treated with concurrent chemoradiotherapy and preventive swallowing exercises. Eur Arch Otorhinolaryngol 2015;272(11):3521-31.

14. Barbon CE, Steele CM. Efficacy of thickened liquids for eliminating aspiration in head and neck cancer: a systematic review. Otolaryngol Head Neck Surg. 2014;152:211-8.

15. Nagaya M, Teruhiko K, Yamada T, Igata A. Videofluorographic study of swallowing in Parkinson's disease. Dysphagia. 1998;13: 95-100.

16. Bird MR, Woodward MC, Gibson EM, Phyland DJ, Fonda D. Asymptomatic swallowing disorders in elderly patients with Parkinson's disease: a description of findings on clinical examination and videofluoroscopy in sixteen patients. Age Ageing. 1994;23:251-4.

17. Nilsson H, Ekberg O, Olsson R, Hindfelt B. Quantitative assessment of oral and pharyngeal function in Parkinson's disease. Dysphagia. 1996;11:144-50. 


\section{Chapter 7}

Summary, general discussion, and future perspectives 

The overall objective of this thesis is to quantitatively characterize and evaluate the presence of oropharyngeal dysphagia (OD) in myotonic dystrophy type 1 (DM1). Several measurement tools were used to that end, with an emphasis on the fiberoptic endoscopic evaluation of swallowing (FEES) procedure given its clinical relevance for OD diagnosis and management. In that context, we examined intra- and interobservers' agreement on FEES measurements. Moreover, we investigated how standardized conditions of swallowing trials as well as patients' characteristics affected the variability of FEES responses. This chapter summarizes the main findings and draws some conclusions, which offer recommendations for future research.

Chapter 1 gives a general introduction to the topics addressed in this thesis. It summarizes the genetic mechanisms of DM1 and the clinical features of the disease. It also describes OD and the tools used to measure swallowing function.

\section{Evaluation of oropharyngeal dysphagia in DM1}

Chapter 2 presents an overview of the swallowing function of patients with DM1 based on a systematic literature review. The search was performed in three electronic databases: PubMed, Embase, and The Cochrane Library. In total 16 studies met the inclusion criteria. External and internal validity of the included articles was evaluated using a 10-item critical appraisal tool to provide guidance for the interpretation of findings and the strength of the evidence. None of the studies fulfilled all the criteria for methodological quality. Few studies reported the level of disease severity in the study population, thereby compromising a generalization of the results. Although the heterogeneity of designs and outcome measures used to assess swallowing across studies prevented a generalized conclusion, some trends were observed. OD was a common symptom in DM1, although it was not frequently reported by the patients if they were not specifically questioned about it. Findings from the swallowing examination were dependent on the particular instrumental tool and outcome measurements used in the study. In general, the most prevalent findings were pharyngeal pooling, decreased pharyngeal contraction amplitude, and reduced upper esophageal sphincter (UES) resting pressure. Further research on the pathophysiological aspects of OD in DM1 was recommended, particularly studies that are well designed and include patients with different levels of disease severity.

\section{Pathophysiology of swallowing in DM1}

Chapter 3 describes a prospective study designed to investigate swallowing function in DM1 patients compared to healthy control subjects. The effect of bolus consistency and disease severity on swallowing function was analyzed. Forty-five patients and ten 
healthy controls underwent a standardized FEES protocol using three bolus consistencies: thin liquid, thick liquid, and bite-size cracker. Clinical severity of the disease was assessed by a neurologist using the Muscular Impairment Rating Scale (MIRS). The results showed that swallowing function was significantly altered in DM1 patients. Piecemeal deglutition and postswallow pharyngeal pooling were identified as important features of $O D$ in this population. The magnitude of the impairment depended on, among other factors, bolus consistency. For instance, inability to clear the bolus from the vallecular space was more prominent with swallows of thick liquid consistency, and the number of swallows per bolus (piecemeal deglutition) was higher with bite-size cracker. When comparing patients in the early stages of the disease (MIRS 2 and 3) with healthy control subjects, significant differences in swallowing function were observed, suggesting that swallowing function might not always be preserved at the beginning of the disease. Moreover, the odds for having a more pathological swallowing outcome increased significantly with the severity of the disease. These results emphasize the importance of performing a detailed swallowing assessment in all DM1 patients, independent of the severity of their disease.

\section{Swallow-related quality of life in DM1}

The cross-sectional observational study presented in chapter $\mathbf{4}$ describes swallowrelated quality of life in DM1 patients and evaluates the correlation between patientreported impact of OD on quality of life (QoL), videofluoroscopy of swallowing (VFS) outcomes, and disease severity. To this end, a questionnaire designed to evaluate the impact of swallowing problems on the health-related QoL (SWAL-QOL) of patients with OD was completed by 75 DM1 patients and 25 healthy control subjects. Comparison of the two groups showed that SWAL-QOL median scores were significantly lower for the DM1 group than for the healthy control group. The swallow-related QoL aspects affected by OD are presented and discussed in the study. Moreover, Spearman's correlation coefficient analysis between the level of muscular impairment and SWALQOL did not reached the level of significance after correction for multiple-testing; i.e., swallow-related QoL did not decrease with the severity of the disease. Swallowing function was evaluated using VFS in a subgroup of 28 patients. The results of VFS showed that swallowing function was significantly impaired in DM1 patients. Despite this fact, the correlation between VFS outcomes and SWAL-QOL scores was found to be inconclusive after correction for multiple-testing. The data suggest that SWAL-QOL provides useful information on how DM1 patients perceive their swallowing impairment, but it cannot be used as the sole indicator of the severity of OD. 


\section{FEES outcome measures}

FEES provides a comprehensive examination of the swallowing mechanism, specifically of the pharyngeal phase of swallowing. The main advantages of this procedure are that it is well tolerated and easy to execute. However, there is no consensus on the outcome measurement scales used to interpret the results of the examination, and studies on the standardization of measurement criteria are scant. Considering the role of FEES interpretation on clinical and therapeutic decisions, a better understanding of the aspects influencing the FEES scoring process may guide us towards a more accurate and reliable measurement technique. The following chapters describe studies targeting two aspects of FEES outcome data: observers' agreement of the outcome measurements; and the analysis of the variability of FEES responses.

Chapter $\mathbf{5}$ describes the investigation of the effect that OD etiology, different observers, and bolus consistency might have on the level of observer agreement for measurements in FEES videos, specifically on agreement reached by independent rating versus consensus panel rating. Sixty patients were included in the study and subdivided into groups. One group had OD due to head and neck cancer $(\mathrm{N}=30)$. The other group had $O D$ as a result of a neurological disease $(N=30)$. All patients underwent a standardized FEES examination and the swallows were analyzed by two blinded observers in randomized order. The rating process was divided in two separate tasks: independent versus consensus panel rating. Observer agreement per task was computed using the linear weighted kappa coefficient. The impact of predictors (OD etiology, bolus consistency, and observer score) on the level of agreement were studied using a Bayesian multilevel model. The data analysis showed no effect of OD etiology (neurological or head and neck oncological origin) on the agreement levels, suggesting that the changes in the swallowing anatomy and physiology of the head and neck oncological group did not influence the rating outcome. Overall the levels of agreement were substantial for all FEES variables $(k>0.61)$. Bolus consistency showed a significant effect on the agreement scores; the interobserver agreement level was lower for thin liquid bolus when compared with the other bolus consistencies. Moreover, the scores given in the consensus panel for exactly the same FEES measurements were slightly different from the scores on the independent rating task. This finding reflects the observers' individual interpretation of the ordinal FEES scoring system, and it may be explained by the imprecise description regarding the exact range of each ordinal level of the rating scale. The slightly higher intrapanel observer agreement level shown in this study suggests that this panel method of scoring FEES exams may improve concordance. However, the agreement levels of independent observers and two separate consensus panels should be further investigated.

Chapter 6 consists of an exploratory study that applied a statistical technique, the group-based trajectory model (GBTM), to find and characterize developmental courses of FEES responses to swallowing challenges. The trajectories were described in terms of composition (size of the cluster, etiology of $O D$ ), level of swallow impairment, and how 
the impairment changed over the swallows. The main objective of the study was to identify subgroups of patients with qualitatively distinct FEES responses. Patients included in the study belonged to one of the three groups created on the basis of OD etiology. In the first group, OD was due to head and neck cancer (HNC) and possible oncological treatment effects on swallowing; in the second group, OD was a consequence of Parkinson's disease (PD); and in the final group, OD was due to DM1. Three bolus consistencies were offered during the seven swallowing trials: thin liquid, thick liquid, and bite-size cracker. For most FEES variables, the trajectories were qualitatively distinct in degree and kind. Highly impaired categories were more stable throughout the different swallow trials with distinct bolus consistencies, whereas intermediate trajectories were more sensitive to changes in bolus consistency. The preliminary data suggest that GBTM was able to capture the qualitatively distinct patterns of FEES responses, revealing regular or irregular patterns or phenotypes of swallowing behaviors that could be linked to different etiologies of OD.

\section{General discussion and future perspectives}

The complexity of DM1 and the heterogeneity of the clinical manifestations complicate the management of the disease. Patients with DM1 manifest a variety of symptoms requiring regular medical assessment and treatment. In the Netherlands, the guideline for assessment and treatment ${ }^{1}$ of DM1 recommends an annual medical check-up of the muscular, cardiac, respiratory, gastrointestinal, and visual systems. That check-up is done by a neurologist or a healthcare professional specialized in DM1. Screening for OD is also recommended. It is based on questions related to symptoms of swallowing impairment such as problems with chewing, cough while eating or drinking, sensation of food remaining in the throat after swallowing, etc. If swallowing complaints and/or recurrent aspiration pneumonia are identified, patients are referred to an ear, nose, and throat (ENT) specialist or to a speech and language pathologist (SLP) for a more detailed swallowing examination. Currently there is no validated checklist or screening tool to investigate OD that is designed specifically for the DM1 population. It is important to consider that a referral for swallowing examination based solely on the information given by the patients may not identify all of those with OD, as the symptoms are usually under-recognized by DM1 patients. ${ }^{2-4}$ However, as this thesis shows, although there is a discrepancy between patients' perception of the impairment and the severity of $O D$ in the clinical examination, DM1 patients do report OD symptoms when specifically asked about these.

When dealing with progressive neuromuscular disorders, it is common clinical practice to refer patients for swallowing assessment based on the severity of the disease despite the absence of symptoms. For DM1, this means that patients with an advanced stage of muscular impairment must be advised to undergo an instrumental swallowing evaluation. This line of reasoning would probably be effective for most DM1 patients 
who present severe muscular impairment, as there is a higher chance they would present OD. However, the association between severity of disease and OD is not clear and the findings in the literature are controversial. ${ }^{5,6}$ In this research, we found that swallowing dysfunction may be present in the early stages of the muscular impairment and saw no significant correlation between severity of muscular impairment and patients' perception of the impact of OD on their QoL. Therefore, to allow early detection of OD in DM1, aspects such as patients' symptoms and disease severity should be combined with information provided by family members and/or caregivers. Furthermore, health care professionals should be vigilant and well instructed to recognize the signs of OD. The development of disease-specific screening is needed, as it would facilitate the identification of DM1 patients with OD and could minimize the complications associated with it.

The studies presented in this thesis offer clinically relevant information on the characteristics of the swallowing mechanism of DM1. The studies revealed that OD is frequently present in this population, compromising oral and pharyngeal phases of swallowing. Contrary to the findings of other studies on dysphagia secondary to neurological diseases such as Parkinson's disease, stroke, etc., the main aspect of OD in DM1 found in this thesis was not aspiration but postswallow pharyngeal pooling. ${ }^{7,8}$ Generally, aspiration has been considered the most important outcome measurement in swallowing examination because it indicates an unsafe oral intake, and increases the risk of developing aspiration pneumonia. ${ }^{9,10}$ The low frequency of aspiration in the swallowing pathophysiology of DM1 patients may give the impression that OD in this population is not severe. However, it has been shown that pharyngeal pooling can be hazardous because the bolus remaining in the pharynx can enter the airway after swallowing anyway. ${ }^{11,12}$ Pharyngeal pooling is an important indicator of swallowing efficiency. Increased pharyngeal pooling represents impaired or incomplete pharyngeal bolus transportation. Accurate instrumental assessment and interpretation of each outcome measure is crucial when describing the phenotype and the severity of OD. Determining the characteristics of OD in DM1 would not only further our understanding of the mechanisms causing the impairment but also help us propose efficient treatment modalities. The challenge remains to develop rehabilitation programs that would focus on the physiological process of the swallowing impairment in DM1.

In line with the well-known variability of the DM1 clinical aspects, this research found that swallowing impairment was more prevalent and more severe in the later stages of the disease but in some cases also present in the early phases. The sample of DM1 evaluated in a cross-sectional study presented different levels of muscular impairment, which allowed us to observe the distinct patterns of swallowing performance related to the severity of the muscular impairment. However, due to the progressive nature and the personal variability of the disease, a longitudinal study would provide more insight into the patterns of swallowing performance and reveal how OD features change in function as the disease progresses. 
In this thesis, swallowing performance of patients with OD was observed to vary according to bolus consistency. That is, DM1 patients presented less postswallow vallecular pooling with bite-size cracker than with thick liquid bolus. These observations suggest a recommendation of bolus modification as a therapeutic strategy to adapt swallowing function in DM1 dysphagic patients. However, there is currently insufficient evidence that DM1 patients would benefit from this sort of treatment in a long run. Well-designed studies investigating the effect of bolus modification on the swallowing efficiency and safety in DM1 patients are needed to support any such recommendations.

It was only a few decades ago that health-related QoL questionnaires were first included in the assessment protocol of patients with OD. Before then, clinicians gave priority to the diagnosis and treatment of the impairment and did not question the impact on patients' QoL. Inclusion of health-related QoL measurements in clinical practice allowed us to elucidate how patients react to the difficulties imposed by the disease and discern to what extent their daily activities are compromised by the impairment. The inclusion of such measurements also allowed clinicians to be more patient-centered and to calibrate clinical actions according to the patients' priorities. Eating, a constant in daily living, comprises not only nutritional but also social aspects, so a swallowing evaluation would not be complete without considering the significance of $O D$ in a patient's life. For this reason, in this thesis the investigation of swallowing physiology was complemented with the assessment of swallow-related quality of life. The investigation showed that both assessments, namely the instrumental evaluation and the patients' reported assessment (SWAL-QOL), are complementary. These findings substantiated our recommendation of a multidimensional assessment as best clinical practice for the evaluation of OD in DM1.

As mentioned before, the discrepancy found between DM1 patients' perception of the impact of OD on QoL and the severity of the impairment identified by the instrumental evaluation was noteworthy. It is not clear whether the lack of correlation between the VFS and SWAL-QOL outcomes were mainly caused by personality characteristics of DM1 patients or by a gradual adaptation of the patients to the swallowing dysfunction during the progression of the muscular impairment. Future research should explore the underlying reasons for the disparity between patients' perception and the severity of the impairment, as the elucidation of those reasons could improve clinical care for these patients. For instance, lack of recognition of the swallowing impairment would probably lead to low compliance with a dysphagia therapy program. It is therefore worth investigating whether specific strategies to increase awareness of the swallowing impairment, such as surface electromyography (sEMG) or FEES biofeedback, could improve the compliance with and effectiveness of the rehabilitation program.

The growing importance of evidence-based medicine has encouraged scientific research on the properties of the measurement tools applied in clinical practice. As became clear from the systematic review of the literature, studies on swallowing in DM1 patients often differed regarding the instrumental examination tools and outcome 
measures. This literature review confirmed the lack of a standardized assessment protocol during instrumental measurements to evaluate the pathophysiology of swallowing in MD patients. Currently, the most commonly used instrumental tools to investigate swallowing function are VFS and FEES. While much has been published on attempts to standardize the procedures and interpretation of VFS, studies regarding FEES outcome measurements are scarce. Knowledge of the psychometric properties and precision of the measurement scales could deepen the understanding of the added value of the outcome measurements for the diagnosis of OD and for the evaluation of treatment effect on OD. Therefore, in this thesis, the measurement scales used to assess swallowing in DM1 patients were further explored to gain a better understanding of the FEES outcome variables. Two aspects were investigated: observers' agreement on the FEES measurements and the heterogeneity of the FEES outcomes within and between patients. It was demonstrated that observers' agreement on FEES measurements can be influenced by the type of rating (independent versus consensus panel) and bolus consistency. Elucidating how exogenous and patient-related factors affect observers' agreement allow us to better understand and improve the FEES measurement scales. For instance, in the training program for observers, special attention to aspects of the scoring task that could influence agreement levels, e.g. training the scoring scale in swallows of different bolus consistency, may ensure higher levels of agreement between observers. Regarding the heterogeneity of FEES responses to swallowing challenges, the exploratory study with the group-based trajectory model (GBTM) showed promising results. It seems that the method may help clinicians to risk-stratify patients by recognizing subgroups in need of special attention in the management of OD. However, these data are preliminary and the study should be replicated. Future studies should have larger sample sizes, recruit healthy control subjects, and include groups with other OD etiologies.

The main conclusions drawn in this thesis may be expressed in the form of a recommendation to conduct a multidimensional swallowing assessment for patients with DM1 based on the variability of the disease and on the diversity of factors involved in the evaluation of OD. The results of such an assessment may offer clinicians a clearer picture of the magnitude of the impairment, thereby helping them establish aims, set priorities, and instate rehabilitation programs that are more efficient. 


\section{References}

1. Richtlijnendatabase. Myotone Dystrofie type 1 [Internet]. Kennisinstituut van Medisch Specialisten. Utrecht (NL) c2012-2017. [cited 2017 July 10].Available from: https://richtlijnendatabase.nl/richtlijn/ myotone_dystrofie_type1/myotone_dystrofie_type_1_-_korte_beschrijving.html

2. Marcon $M$, Briani $C$, Ermani M, Menegazzo E, lurilli V, Feltrin GP, Novelli G, Gennarelli M, Angelini C. Positive correlation of CTG expansion and pharyngoesophageal alterations in myotonic dystrophy patients. Ital J Neurol Sci. 1998;19(2):75-80.

3. Leonard RJ, Kendall KA, Johnson R, McKenzie S. Swallowing in myotonic muscular dystrophy: a videofluoroscopic study. Arch Phys Med Rehabil. 2001;82(7):979-85.

4. Eckardt VF, Nix W, Kraus W, Bohl J. Esophageal motor function in patients with muscular dystrophy. Gastroenterology. 1986;90(3):628-35.

5. Ertekin C, Yuceyar N, Aydogdu I, Karasoy H. Electrophysiological evaluation of oropharyngeal swallowing in myotonic dystrophy. J Neurol Neurosurg Psychiatr. 2001;70(3):363-71.

6. Costantini M, Zaninotto G, Anselmino M, Marcon M, lurilli V, Boccu C, Feltrin GP, Angelini C, Ancona E. Esophageal motor function in patients with myotonic dystrophy. Dig Dis Sci. 1996;41(10):2032-8.

7. Logemann JA, Gensler G, Robbins J, Lindblad AS, Brandt D, Hind JA, et al. A randomized study of three interventions for aspiration of thin liquids in patients with dementia or Parkinson's disease. J Speech Lang Hear Res. 2008;51:173-83

8. Suntrup S, Kemmling A, Warnecke T, Hamacher C, Oelenberg S, Niederstadt T, Heindel W, Wiendl H, Dziewas R. he impact of lesion location on dysphagia incidence, pattern and complications in acute stroke. Part 1: dysphagia incidence, severity and aspiration Eur J Neurol. 2015;22(5):832-8.

9. Eisenhuber E, Schima W, Schober E, Pokieser P, Stadler A, Scharitzer M, et al. Videofluoroscopic assessment of patients with dysphagia: pharyngeal retention is a predictive factor for aspiration. AJR Am J Roentgenol. 2002;178:393-8.

10. Langmore SE, Terpenning MS, Schork A, et al. Predictors of aspiration pneumonia: how important is dysphagia? Dysphagia 1998;13:69-81

11. Feinberg MJ, Ekberg O. Videofluoroscopy in elderly patients with aspiration: importance of evaluating both oral and pharyngeal stages of deglutition. AJR 1991;156:293-6.

12. Molfenter SM, Steele CM. The relationship between residue and aspiration on the subsequent swallow: an application of the normalized residue ratio scale. Dysphagia. 2013;28(4):494-500. 
Nederlandse samenvatting 



\section{Nederlandse samenvatting}

De algemene doelstelling van dit proefschrift is om de aanwezigheid van orofaryngeale dysfagie (OD) bij myotone dystrofie type 1 (DM1) patiënten kwantitatief te beschrijven en te evalueren. Hiervoor werden verschillende meetinstrumenten gebruikt, met de nadruk op endoscopische slikvideo (fiberoptic endoscopic evaluation of swallowing FEES) vanwege de klinische relevantie van FEES bij de diagnose stelling en behandeling van OD. In dit kader hebben we de intra- en interbeoordelaarsovereenstemming van FEES metingen onderzocht. Daarnaast hebben we onderzocht wat de invloed was van gestandaardiseerde onderzoeksvoorwaarden tijdens de sliktrials en van patiëntkarakteristieken op de variabiliteit van FEES uitkomsten. In dit hoofdstuk worden de belangrijkste bevindingen samengevat en conclusies beschreven die behulpzaam kunnen zijn voor toekomstig wetenschappelijk onderzoek.

Hoofdstuk 1 geeft een algemene introductie over de onderwerpen die in dit proefschrift aan bod komen. Een overzicht van de onderliggende genetische mechanismen van DM1 en de klinische kenmerken van deze ziekte worden beschreven. In dit hoofdstuk worden eveneens het concept OD en de instrumenten om de slikfunctie te meten beschreven.

\section{Evaluatie van orofaryngeale dysfagie in DM1}

Hoofdstuk 2 geeft een overzicht van de slikfunctie van patiënten met DM1 op basis van een systematisch literatuuronderzoek. De zoekactie werd uitgevoerd in drie elektronische bibliografische databases: PubMed, Embase en The Cochrane Library. In totaal voldeden 16 studies aan de inclusiecriteria. De externe en interne validiteit van de geïncludeerde artikelen werd geëvalueerd middels een kritisch beoordelingsinstrument bestaande uit 10 items om richting te geven aan de interpretatie van de studie bevindingen en de sterkte van de wetenschappelijke bewijslast. Geen enkele studie voldeed aan alle criteria voor methodologische kwaliteitstoetsing. Weinig studies rapporteerden de mate van ernst van de ziekte (DM1) in de studiepopulatie, waardoor het generaliseren van de studie resultaten niet mogelijk was.

Hoewel de heterogeniteit van de studie ontwerpen en verschillende uitkomstmaten, die gebruikt werden om de slikfunctie in de verschillende studies te bepalen, het vormen van een algemene conclusie belemmerden, werden toch enkele trends gezien. OD was een veel voorkomend symptoom in DM1, hoewel OD niet vaak gemeld werd door patiënten wanneer er niet specifiek naar gevraagd werd. De bevindingen van het slikonderzoek per geïncludeerde studie waren afhankelijk van het specifieke meetinstrument en de uitkomstmaten die gebruikt werden in het onderzoek. Over het algemeen waren de meest voorkomende bevindingen faryngeale stase, verminderde amplitude van de faryngeale contractie en een verminderde rustdruk in de bovenste 
slokdarmsfincter (UES). Vervolg wetenschappelijk onderzoek naar de pathofysiologische aspecten van OD in DM1 is noodzakelijk, met name high-quality methodologische studies met patiënten die zich bevinden in verschillende ziekte stadia van DM1.

\section{Pathofysiologie van de slikfunctie bij DM1}

Hoofdstuk 3 beschrijft een prospectieve studie die opgezet is om de slikfunctie van DM1 patiënten te onderzoeken in vergelijking met gezonde controle personen. Het effect van bolusconsistentie en de ernst van de ziekte op de slikfunctie werd geanalyseerd. Vijfenveertig patiënten en tien gezonde controle personen ondergingen een gestandaardiseerd FEES protocol waarbij gebruik werd gemaakt van drie bolusconsistenties: dun vloeibaar, dik vloeibaar en een hapklare mini cracker. De klinische ernst van de ziekte werd beoordeeld door een neuroloog aan de hand van de Muscular Impairment Rating Scale (MIRS). De resultaten toonden aan dat de slikfunctie significant veranderd was bij DM1 patiënten. Het toegenomen aantal slikacties per bolus en faryngeale stase werden geïdentificeerd als belangrijke kenmerken van OD in deze populatie. De ernst van de gemeten slikdisfunctie was onder andere afhankelijk van bolusconsistentie. Bijvoorbeeld het onvermogen om bolus vanuit de vallecula te verwijderen was meer prominent zichtbaar tijdens het slikken van dik vloeibare consistentie en het aantal slikacties per bolus was hoger bij het doorslikken van een cracker. Bij het vergelijken van patiënten in een vroeg ziekte stadium (MIRS 2 en 3) met gezonde controle personen werden significante verschillen in slikfunctie gezien, wat suggereert dat de slikfunctie mogelijk niet altijd behouden blijft in het beginstadium van de ziekte. Bovendien nam de kans op een meer pathologische slikuitkomst significant toe bij toename van de ziekte ernst. Deze resultaten benadrukken het belang van het uitvoeren van een gedetailleerd slikonderzoek bij alle DM1 patiënten onafhankelijk van de ernst van de ziekte.

\section{Slik-gerelateerde kwaliteit van leven bij DM1}

De cross-sectionele observationele studie weergegeven in hoofdstuk 4 beschrijft de slik-gerelateerde kwaliteit van leven van DM1 patiënten en evalueert de correlatie tussen de patiënt-gerapporteerde invloed van OD op de kwaliteit van leven (QoL), uitkomstmaten van videofluoroscopische slikevaluatie (VFS) en de ernst van de ziekte. De SWAL-QOL, een vragenlijst ontworpen om de invloed van slikproblemen op de gezondheid-gerelateerde QoL te evalueren, werd ingevuld door 75 DM1 patiënten en 25 gezonde controle personen. Vergelijking van de twee groepen toonde aan dat de mediane SWAL-QOL scores significant lager waren voor de DM1 groep dan de scores van de gezonde controle groep. De aspecten aangaande slik-gerelateerde QoL die beïnvloed worden door OD worden weergegeven en besproken in deze studie. Spearman's correlatiecoëfficiënt analyse behaalde na correctie voor multiple testing niet het significantieniveau om een correlatie tussen de mate van spierdisfunctie en de 
SWAL-QOL score te vinden, d.w.z. scores van de slik-gerelateerde QoL namen niet af met de ernst van de ziekte. De slikfunctie werd geëvalueerd middels VFS in een subgroep bestaande uit 28 patiënten. De resultaten van de VFS toonden een significant verslechterde slikfunctie in DM1 patiënten. Ondanks deze bevinding bleek de correlatie tussen VFS uitkomsten en SWAL-QOL scores niet eenduidig te zijn na correctie voor multiple testing. De gegevens suggereren dat de SWAL-QOL nuttige informatie verschaft over de manier waarop DM1 patiënten een verminderde slikfunctie ervaren, maar dat de SWAL-QOL niet kan worden gebruikt als enige indicator om de ernst van OD te bepalen.

\section{FEES uitkomstmaten}

FEES biedt de mogelijkheid tot een uitgebreid onderzoek van het slikmechanisme met name van de faryngeale slikfase. De voornaamste voordelen van deze procedure zijn dat het FEES onderzoek goed verdragen wordt en gemakkelijk uit te voeren is. $\mathrm{Er}$ bestaat echter geen consensus over de schalen van uitkomstmaten aan de hand waarvan de resultaten van het onderzoek geïnterpreteerd worden en er zijn weinig studies omtrent de standaardisatie van de meetcriteria. Wat betreft het belang van FEES interpretatie ten aanzien van klinische en therapeutische beslissingen, zou een beter begrip van de aspecten die het proces van het scoren van FEES onderzoeken beïnvloeden, ons kunnen leiden naar een accuratere en meer betrouwbare meettechniek. De volgende hoofdstukken beschrijven studies die zich richten op twee aspecten van FEES uitkomstdata: beoordelaarsovereenstemming van de uitkomst metingen en de analyse van de variabiliteit van FEES resultaten.

Hoofdstuk 5 beschrijft het onderzoek naar het effect dat OD etiologie, verschillende beoordelaars en bolusconsistentie zouden kunnen hebben op de mate van beoordelaarsovereenstemming voor metingen in FEES video's en meer specifiek op de mate van overeenstemming bereikt tijdens onafhankelijke individuele scoring vergeleken met scoring door een consensus panel. Zestig patiënten werden geïncludeerd in de studie en onderverdeeld in groepen. Een groep leed aan OD ten gevolge van hoofd-hals kanker $(\mathrm{N}=30)$. De andere groep had OD als gevolg van een neurologische ziekte $(\mathrm{N}=30)$. Alle patiënten ondergingen een gestandaardiseerd FEES onderzoek en de slikacties werden in gerandomiseerde volgorde geanalyseerd door twee geblindeerde beoordelaars. Het beoordelingsproces werd verdeeld in twee aparte taken: onafhankelijke individuele beoordeling versus beoordeling middels consensus door een panel. De mate van beoordelaarsovereenstemming per taak werd berekend met behulp van lineair gewogen kappa coëfficiënt. De invloed van voorspellende factoren (OD etiologie, bolusconsistentie en beoordelaarsscore) op de mate van overeenstemming werd geanalyseerd middels een Bayesiaans multilevel model. De data analyse toonde geen effect van OD etiologie (neurologische of hoofd-hals oncologische origine) op de mate van overeenstemming wat suggereert dat 
veranderingen in de slikanatomie en fysiologie bij de hoofd-hals oncologische groep geen invloed hadden op de toegekende scores. Over het algemeen was de mate van beoordelaarsovereenstemming substantieel voor alle FEES variabelen $(\kappa>0.61)$. Bolusconsistentie vertoonde een significant effect op de mate van overeenstemming; de mate van interbeoordelaarsovereenstemming was lager voor dun vloeibare bolus vergeleken met andere bolusconsistenties. Bovendien verschilden de scores toegekend door het consensus panel iets van de scores tijdens de individuele taak voor exact dezelfde FEES metingen. Deze bevinding weerspiegelt de individuele interpretatie van het ordinale FEES scoringssysteem door de beoordelaar en dit kan mogelijk verklaard worden door de onnauwkeurige omschrijving van de exacte omvang van ieder ordinaal niveau van de beoordelingsschaal. De iets hogere score op de intrapanel beoordelaarsovereenstemming zoals getoond in deze studie suggereert dat deze panel methode om FEES onderzoeken te beoordelen de overeenstemming zou kunnen verbeteren. De mate van overeenstemming tussen onafhankelijke beoordelaars en tussen twee aparte consensus panels dient verder onderzocht te worden.

Hoofdstuk 6 bevat een verkennend onderzoek waarin een statistische techniek genaamd group-based trajectory model (GBTM) werd toegepast om het verloop van trajecten oftewel de ontwikkeling van FEES uitkomsten tijdens slik-gerelateerde uitdagingen of taken aan te tonen en te beschrijven. De zogenaamde trajecten werden beschreven in termen van samenstelling (grootte van het cluster, etiologie van OD in dat cluster), ernst van de slikdisfunctie en de manier waarop de slikdisfunctie veranderde gedurende een aantal sliktrials. Het voornaamste doel van de studie was het identificeren van subgroepen van patiënten die gekenmerkt werden door kwalitatief te onderscheiden FEES uitkomsten. Patiënten die geïncludeerd werden in de studie behoorden tot een van de drie groepen die gecreëerd waren op basis van OD etiologie. In de eerste groep was OD het gevolg van hoofd-hals kanker en mogelijke gevolgen van oncologische behandeling op de slikfunctie $(\mathrm{N}=73)$; in de tweede groep was $O D$ het gevolg van de ziekte van Parkinson $(\mathrm{N}=89)$ en in de laatste groep was $O D$ het gevolg van DM1 $(\mathrm{N}=43)$. Drie bolusconsistenties werden toegediend gedurende zeven sliktrials: dun vloeibaar, dik vloeibaar en een hapklare mini cracker. Voor de meeste FEES variabelen waren de trajecten kwalitatief onderscheidend in ernst en aard van de OD. Ernstig aangedane categorieën van ordinale FEES variabelen waren stabieler gedurende de verschillende sliktrials met verschillende bolusconsistenties terwijl intermediaire trajecten gevoeliger waren voor veranderingen in bolusconsistentie. Deze preliminaire data suggereren dat GBTM in staat was om kwalitatief onderscheidende patronen in de FEES uitkomsten te herkennen waarbij regelmatige of onregelmatige patronen of fenotypes van slikgedrag die gelinkt kunnen worden aan verschillende etiologieën van $O D$ onthuld werden. 
Valorization 



\section{Valorization}

Valorization is defined as "the process of creating value from knowledge, by making knowledge suitable and/or available for social (and/or economic) use and by making knowledge suitable for translation into competitive products, services, processes, and new commercial activities" (National Valorization Committee). The studies presented in this thesis were intended to help determine the characteristics of oropharyngeal dysphagia (OD) in myotonic dystrophy type 1 (DM1), and to understand and subsequently improve the clinimetric characteristics of the fiberoptic endoscopic evaluation of swallowing (FEES) scales.

$O D$, which involves difficulty with eating and drinking, is a disorder that affects over 16 million people in the USA and over 40 million in Europe. ${ }^{1}$ It is a common symptom in neurological diseases such as stroke, Parkinson's, Alzheimer's, and DM1. Its complications may include malnutrition, dehydration, and aspiration pneumonia; in DM1 patients, pneumonia is reported as one of the most frequent primary causes of mortality. ${ }^{2}$

OD also takes a heavy financial toll on the healthcare system. In 2010 the economic impact of dysphagia in hospital settings in the USA was calculated to be USD 547 million annually. ${ }^{3}$ That financial burden is linked to the extended length of hospitalization, higher rate of respiratory infection, increased expenditure on medical equipment and treatment, e.g. enteral feeding, antibiotics, etc., that are associated with dysphagia. While the cost of treating OD can be quantified, the social and psychological burden that significantly reduces quality of life for both patients and caregivers is more difficult to evaluate. Until 2010, there were 1117 patients with myotonic dystrophy registered in the Netherlands. The exact prevalence of OD in these population is not known; the literature worldwide puts it in the range of 25 to $80 \% .^{4-6}$ Despite the importance of preventing its associated complications, $O D$ is often under-diagnosed, presumably because it is overlooked in the clinical assessment of DM1 patients.

Professionals from several disciplines are involved in the assessment and management of dysphagia: medical specialists, speech-language pathologists, nurses, dieticians, and caregivers. Because dysphagia requires a multidisciplinary approach, the results of our research are of relevance to all these professions. Therefore, we recommend a multidimensional assessment to evaluate swallowing in DM1. The underlying idea is that early and precise identification of OD may prevent complications that would reduce personal health-related quality of life and increase healthcare costs.

Diagnostics and treatment of OD in DM1 frequently rely on low to moderate levels of evidence, as compared to the literature available to cardiologists or neurologists, for instance. In our opinion, the paucity of well-designed studies in dysphagic DM1 patients cannot be attributed to disinterest on the part of patients as well as the medical community. Rather, the current state of knowledge apparently reflects the scarcity of financial resources for basic research in this field, as most of governmental and privatesector funding favors research with societal and economic applications. We do not 
dispute the importance of knowledge valorization, i.e., making knowledge suitable or available for social and/or economic use. Yet we do question the feasibility of evidencebased practice in the healthcare and medical sectors without support from basic and fundamental research.

The studies presented in this thesis do not directly address the implementation of an assessment procedure or a specific treatment modality for OD. All our studies could be classified as 'basic or exploratory research' and, as such, their societal impact is more difficult to establish. We do believe, however, that in the longer term they will serve as stepping stones, paving the way for future studies on the implementation of diagnosis and/or disease-specific guidelines for identification of OD in DM1 patients. A more refined understanding of the psychometric characteristic of FEES scales, of the underlying pathophysiology of dysphagia in DM1 as well as of patients' perception of the swallowing impairment are fundamental to the development of disease-specific guidelines and different treatment modalities specifically for this population. 


\section{References}

1. Rofes L, Arreola V, Almirall J, Cabré M, Campins L, García-Peris P, et al. Diagnosis and management of oropharyngeal dysphagia and its nutritional and respiratory complications in the elderly. Gastroenterol Res Pract. 2011.

2. de Die-Smulders CE, Höweler CJ, Thijs C, Mirandolle JF, Anten HB, Smeets HJ, Chandler KE, Geraedts JP. Age and causes of death in adult-onset myotonic dystrophy. Brain.1998;121(Pt 8):1557-63.

3. Altman KW, Yu GP, Schaefer SD Consequence of dysphagia in the hospitalized patient: impact on prognosis and hospital resources. Arch Otolaryngol Head Neck Surg. 2010;136(8):784-9

4. Bellini M, Biagi S, Stasi C, Costa F, Mumolo MG, Ricchiuti A, Marchi S. Gastrointestinal manifestations in myotonic muscular dystrophy. World J Gastroenterol. 2006;8;12(12):1821-8.

5. Modolell I, Mearin F, Baudet JS, Gamez J, Cervera C, Malagelada JR Pharyngo-esophageal motility disturbances in patients with myotonic dystrophy. Scand J Gastroenterol. 1999;34(9):878-82.

6. Ertekin C, Yuceyar N, Aydogdu I, Karasoy H. Electrophysiological evaluation of oropharyngeal swallowing in myotonic dystrophy. J Neurol Neurosurg Psychiat. 2001;70(3):363-71. 

Acknowledgements 



\section{Acknowledgements}

The development of this research has been a period of intense learning for me, not only in the scientific aspect, but also on a personal level. I would like to thank each and every one who supported and guided me throughout this period and specially some people.

The supervisor: Prof. dr. Kremer. Dear Bern, your valuable suggestions on the articles were very much appreciated. You could always bring a broader perspective into the subject. Thanks for accepting me in your group and for giving the support to develop this line of research in the ENT department under your guidance.

The co-supervisors: There are two persons that I cannot thank enough. In fact, during the whole PhD trajectory I always got the impression that I've got two god-mothers disguised as co-supervisors:

Dr. Baijens. Dear Laura, you have played a major role in my academic development. Thank you for taking me under your wing and for providing me numerous opportunities to learn and develop. I could not wish for a better co-supervisor. Your endless energy is impressive, very quick in replying my emails, revising the manuscript with an 'eagle eye' and for always bringing a positive perspective in the difficult moments. The joy and enthusiasm you have for your work are contagious and motivational to me. This thesis would not have been possible without your extraordinary support.

Dr. Lima Passos. Dear Valeria. You are much more than a supervisor, you are a dear friend. Having you at my side during this challenging path was a real gift. Your support went beyond the academic work. Thank you for always being available to answer my uncountable questions and for making statistics sounds a bit less complicated. You support, thrust and friendship were an important motor that kept me going through the PhD.

Nel Roodenburg: Dear Nel, when I had almost lost hope of working with dysphagia in the Netherland I met you and you open the doors of dysphagia clinical work to me. You were the first person to encourage me to do the PhD and for this I'll be always grateful. I thank you for all the time and effort you dedicated collecting the data for this research and putting me in contact with the patients.

Prof. dr. Karin Faber. Dear Karin, Thank you for your support in providing me the data on severity of the muscular impairment of the myotonic dystrophy patients used in this thesis and for your valuable comments on the articles. I hope this partnership can grow even stronger in the future. 
Dr. Vanbelle. Dear Sophie, thank you for your support in the statistical analysis of the paper 'Observers' agreement on measurements in fiberoptic endoscopic evaluation of swallowing'.

The raters: R. Verdonschot, J Meijers, M. van Hooren, T. van Becelaere, F Wesseling. Dear Rob, Jolanda, Michel, Tine and Frederik. Thank you for your patience and for all the hours you expended in front of the computer, rating the swallowing videos over and over again. Without your precious support it would not be possible to conduct this research.

Even after all these years living in the Netherlands writing the 'samenvatting' section of this thesis was beyond my capacity. Thanks to Dr. Laura Baijens, Sorina Simons and Dirce Fleming for the translation and revision of the text.

I'd like to express my gratitude to the staff secretaries from the KNO department for their assistance and help. A special thanks goes to Ester Moesen-Paquay for arranging all the paperwork and guiding me through the end of the PhD program.

I would like to thank my assessment committee members: Prof. dr. Pere Clave, Dr. Lisette van der Molen, Prof. dr. Simon Robben, Prof. dr. Jeroen Vermeulen for their time, interest, and helpful comments. I would also like to thank the other members of my oral defense committee, Prof. dr. Bing Tan, Prof. dr. Karin Faber and Prof. dr. Nathalie Rommel.

A big "Thank you!" also goes out to everybody who participated in this study including all my friends who helped me to collect the data in healthy subjects.

Nobody has been more important to me in the pursuit of this project than the members of my family. I would like to thank my parents, Walter and Maria Helena who spared no effort to provide the best possible environment for me to grow up. Your love and guidance are always with me.

A very special thanks goes to the basic source of my energy: my husband Vicente and my daughters Marina and Gabriela. You have cherished with me every great moment and supported me when I needed it. Your presence in my life give me strength and fills me with love. Estar com vocês é tudo de bom! 
Curriculum vitae 



\section{Curriculum vitae}

Walmari Pilz was born on the 11th of November in Santa Rosa in the state of Rio Grande do Sul, Brazil. After graduating from high school, she moved to Porto Alegre where she attended the Federal University of Rio Grande do Sul and graduated in music in 1987. She worked as a music teacher for some years, during which time she studied speech-language pathology in the Methodist Institute of Education and Culture and received her bachelor diploma in 1995. In 1999, after following a post graduate course for two years, she received her certification as a specialist in oral motor disorders. In Brazil she worked for 10 years as an SLP in the private and public health sectors, performing evaluation and treatment of patients with dysphagia and/or communication disorders. Her clinical expertise resulted in invitations for participation as a lecturer in courses and conferences in Brazil.

In 2005, she moved to the Netherlands with her husband Vicente and her two daughters, Marina and Gabriela. Her interest in the topic 'oropharyngeal dysphagia' led her to a collaboration in the research developed by Dr. Baijens at Maastricht University Medical Center. This collaboration resulted in the publication of scientific papers in peer-reviewed journals. In 2012 she started her PhD project on oropharyngeal dysphagia in myotonic dystrophy type 1 at Maastricht University Medical Center. Since 2016 she has a part-time position as a researcher in the Department of Otorhinolaryngology, Head and Neck Surgery of the Maastricht University Medical Center where she is responsible for data management, grant application and cosupervision of research projects on oropharyngeal dysphagia. 

List of publications 



\section{List of publications}

Pilz W, Passos VL, Verdonschot R, Meijer J, Roodenburg N, Faber CC, Kremer B, Baijens LW. Swallow-related quality of life and oropharyngeal dysphagia in myotonic dystrophy. Submitted

Pilz W, Vanbelle S, Passos VL, Kremer B, van Hooren M, Becelaere T, Roodenburg N, Baijens LW Observers' agreement on measurements in fiberoptic endoscopic evaluation of swallowing. Dysphagia. 2016;31(2):180-7.

Baijens LW, Pilz W, Kremer B, Passos VL. Identifying Patterns of FEES-Derived Swallowing Trajectories Using Group-Based Trajectory Model. Dysphagia. 2015;30(5):529-39

van Hooren MR, Baijens LW, Vos R, Pilz W, Kuijpers LM, Kremer B, Michou E. Voice- and swallow-related quality of life in idiopathic Parkinson's disease. Laryngoscope. 2016;126(2):408-14

Pilz W, Baijens LW, Passos VL, Verdonschot R, Wesseling F, Roodenburg N, Faber CC, Kremer B Swallowing assessment in myotonic dystrophy type 1 using fiberoptic endoscopic evaluation of swallowing (FEES). Neuromuscul Disord. 2014;24(12):1054-62.

Baijens LW, Speyer R, Pilz W, Roodenburg N. FEES protocol derived estimates of sensitivity: aspiration in dysphagic patients. Dysphagia. 2014;29(5):583-90.

Pilz W, Baijens LW, Kramer B. Pathophysiology of swallowing in patients with myotonic dystrophy type 1: A Systematic Review. Dysphagia. 2014 Jun;29(3):319-31.

Baijens LW, Barikroo A, Pilz W. Intrarater and interrater reliability for measurements in videofluoroscopy of swallowing. Eur J Radiol. 2013 Oct;82(10):1683-95.

Baijens LW, Speyer R, Passos VL, Pilz W, van der Kruis J, Haarmans S, DesjardinsRombouts C. Surface electrical stimulation in dysphagic Parkinson patients: a randomized clinical trial. Laryngoscope. 2013 Nov;123(11):E38-44

Baijens LW, Koetsenruijter K, Pilz W. Diagnosis and treatment of phagophobia: a review. Dysphagia. 2013 Jun;28(2):260-70.

Baijens LW, Speyer R, Passos VL, Pilz W, Roodenburg N, Clavé P. The effect of surface electrical stimulation on swallowing in dysphagic Parkinson patients. Dysphagia. 2012 Dec;27(4):528-37. 
Baijens L, Speyer R, Lima Passos V, Pilz W, Roodenburg N, Clavé P. Swallowing in Parkinson patients versus healthy controls: Reliability of measurements in videofluoroscopy. Gastroenterol Res Pract. 2011; 2011: 380682. Published online 2011 October 3.

Speyer R, Pilz W, van der Kruis J, Brunings JW. Reliability and validity of peer assessment in medical education: A systematic review. Med Teach. 2011;33(11):e57285.

Fischer GB, Pilz W. Aspiration syndromes in children - In: J.S. Jacobi, D.S. Levy, L.M.C. Silva (Eds.), Dysphagia - Evaluation and treatment. 2003 (pp123-133) Rio de Janeiro: Revinter. Portuguese.

Scalco MG, Pimentel RM, Pilz W. Vocal profile of private elementary school teachers. Pró Fono. 1996; 8(2), 25-30. Portuguese 


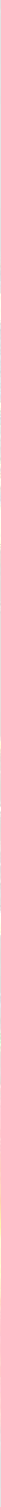

